Studies on Highly Stereoselective Addition-Elimination Reactions of

3-(Methoxycarbonyl)-1,2-allen-4-ols with MX. An Efficient Synthesis of

3-(Methoxycarbonyl)-2-halo-1,3(Z)-dienes

Youqian Deng, Xin Jin, and Shengming $\mathrm{Ma}^{*}$

Laboratory of Molecular Recognition and Synthesis, Department of Chemistry,

Zhejiang University, Hangzhou 310027, Zhejiang, P.R. China

masm@mail.sioc.ac.cn

Supporting Information

\begin{tabular}{|l|l|}
\hline Analytical data for all the products listed in the text & S2 \\
\hline${ }^{1}$ H NMR and ${ }^{13}$ C NMR Spectra of those compounds & S24 \\
\hline
\end{tabular}




\section{Synthesis of 3-(methoxycarbonyl)-1,2-allen-4-ols}

\section{(1) 3-(Methoxycarbonyl)-1,2-hexadien-4-ol (3a). Typical Procedure. ${ }^{1}$}

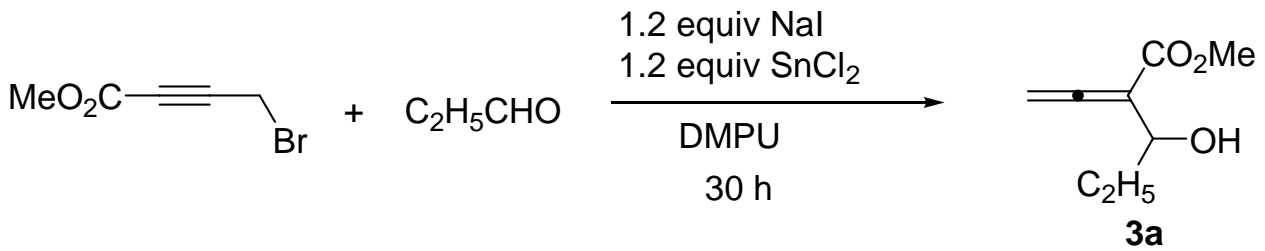

To a solution of 3-(methoxycarbonyl)propargyl bromide (3.4806 g, $19.7 \mathrm{mmol})^{2}$ in $30 \mathrm{~mL}$ of DMPU were added $\mathrm{SnCl}_{2}(4.6011 \mathrm{~g}, 24.2 \mathrm{mmol})$ and $\mathrm{NaI}$ (3.6107 g, 24.1 mmol) at rt. The resulting yellow slurry was stirred in the absence of light (the reaction tube was wrapped with a black plastic bag) for 6 hours. A solution of propionaldehyde (0.9281 g, $16.0 \mathrm{mmol})$ in $10 \mathrm{~mL}$ of DMPU was then added dropwise over 15 min at $0{ }^{\circ} \mathrm{C}$. The orange reaction mixture was allowed to warm up to room temperature and stirred in dark for an additional 24 hours. The reaction was cooled to $0{ }^{\circ} \mathrm{C}$, diluted with $\mathrm{Et}_{2} \mathrm{O}$, and quenched with $30 \mathrm{~mL}$ of saturated aqueous $\mathrm{NH}_{4} \mathrm{Cl}$. The organic phase was separated and the aqueous phase was extracted with $\mathrm{Et}_{2} \mathrm{O}(4 \times 30$ $\mathrm{mL})$. The combined organic layer was washed with brine and dried over anhydrous $\mathrm{Na}_{2} \mathrm{SO}_{4}$. Evaporation and column chromatography on silica gel (petroleum ether/ethyl acetate $=5: 1)$ afforded 3a (1.4606 g, 59\%): Liquid; ${ }^{1} \mathrm{H}$ NMR (400 MHz, $\left.\mathrm{CDCl}_{3}\right) \delta$ $5.20(\mathrm{~d}, J=2.0 \mathrm{~Hz}, 2 \mathrm{H}), 4.32-4.24(\mathrm{~m}, 1 \mathrm{H}), 3.70(\mathrm{~s}, 3 \mathrm{H}), 3.20-2.94$ (bs, $1 \mathrm{H})$, 1.66-1.57 (m, 2H), 0.89 (t, $J=7.4 \mathrm{~Hz}, 3 \mathrm{H}) ;{ }^{13} \mathrm{C}$ NMR (100 MHz, $\left.\mathrm{CDCl}_{3}\right): \delta 212.4$, 167.3, 102.6, 80.5, 70.4, 52.1, 28.2, 10.0; MS (m/z): $156\left(\mathrm{M}^{+}, 0.12\right), 127$ (100); IR (neat, $\mathrm{cm}^{-1}$ ): 3490, 2965, 1964, 1935, 1713, 1266. HRMS calcd for $\mathrm{C}_{8} \mathrm{H}_{12} \mathrm{NaO}_{3}$ $\left(\mathrm{M}^{+}+\mathrm{Na}\right)$ : 179.0679, Found: 179.0676. 
(2) 3-(Methoxycarbonyl)-1,2-heptadien-4-ol (3b)

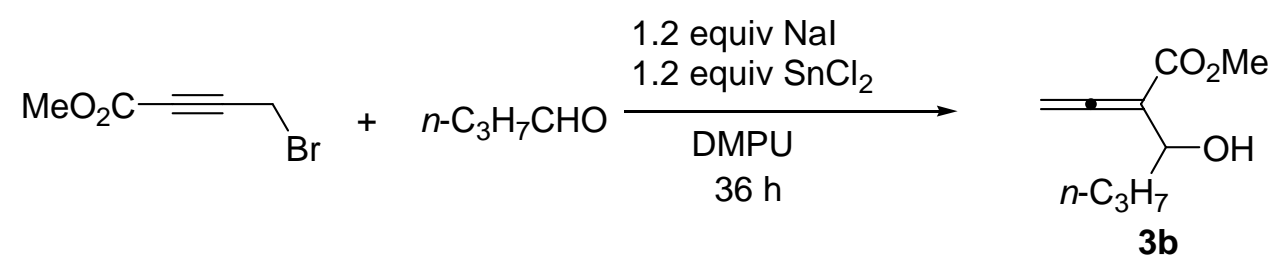

The reaction of $1.7901 \mathrm{~g}$ (10.1 mmol) of 3-(methoxycarbonyl)propargyl bromide, $2.2896 \mathrm{~g}(12.1 \mathrm{mmol})$ of $\mathrm{SnCl}_{2}, 1.8112 \mathrm{~g}(12.1 \mathrm{mmol})$ of $\mathrm{NaI}$, and $0.5702 \mathrm{~g}$ of n-butanal (7.9 mmol) in $22 \mathrm{~mL}$ of DMPU afforded $0.8223 \mathrm{~g}(61 \%)$ of $3 \mathbf{b}$ : Liquid; ${ }^{1} \mathrm{H}$ NMR (400 MHz, $\mathrm{CDCl}_{3}$ ) $\delta 5.22$ (d, $\left.J=2.0 \mathrm{~Hz}, 2 \mathrm{H}\right), 4.41-4.36$ (m, 1H), $3.73(\mathrm{~s}, 3 \mathrm{H})$, 3.00-2.68 (bs, 1H), 1.63-1.55 (m, 2H), 1.48-1.36 (m, 1H), 1.35-1.23 (m, 1H), 0.89 (t, $J=7.2 \mathrm{~Hz}, 3 \mathrm{H}) ;{ }^{13} \mathrm{C}$ NMR $\left(100 \mathrm{MHz}, \mathrm{CDCl}_{3}\right): \delta 212.4,167.4,102.9,80.5,68.8,52.1$, 37.3, 18.9, 13.7; MS (m/z): $170\left(\mathrm{M}^{+}, 0.12\right), 127$ (100); IR (neat, $\left.\mathrm{cm}^{-1}\right)$ : 3479, 2958, 1964, 1939, 1713, 1259. HRMS calcd for $\mathrm{C}_{9} \mathrm{H}_{14} \mathrm{NaO}_{3}\left(\mathrm{M}^{+}+\mathrm{Na}\right)$ : 193.0835, Found: 193.0837.

(3) 3-(Methoxycarbonyl)-5-methyl-1,2-hexdien-4-ol (3c)

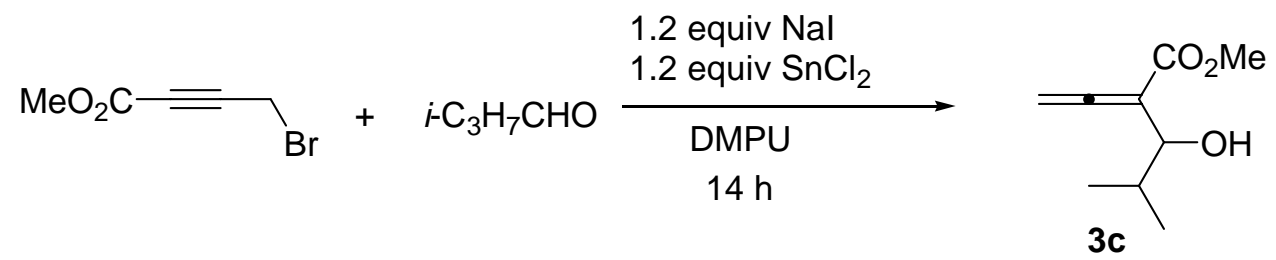

The reaction of $1.0721 \mathrm{~g}(6.1 \mathrm{mmol})$ of 3-(methoxycarbonyl)propargyl bromide, $1.3764 \mathrm{~g}(7.3 \mathrm{mmol})$ of $\mathrm{SnCl}_{2}, 1.0797 \mathrm{~g}(7.2 \mathrm{mmol})$ of $\mathrm{NaI}$, and $0.3564 \mathrm{~g}$ of isobutanal (5.0 mmol) in $15 \mathrm{~mL}$ of DMPU afforded $0.7017 \mathrm{~g}$ (83\%) of 3c: Liquid; ${ }^{1} \mathrm{H}$ NMR (400 MHz, $\left.\mathrm{CDCl}_{3}\right) \delta 5.23(\mathrm{~d}, J=1.2 \mathrm{~Hz}, 2 \mathrm{H}), 4.04\left(\mathrm{dt}, J_{1}=7.6 \mathrm{~Hz}, J_{2}=1.2 \mathrm{~Hz}\right.$, 1H), 3.74 (s, 3H), 2.97-2.69 (bs, 1H), 1.97-1.88 (m, 1H), 0.98 (d, $J=6.4 \mathrm{~Hz}, 3 \mathrm{H}$ ), 
$0.88(\mathrm{~d}, J=6.4 \mathrm{~Hz}, 3 \mathrm{H}) ;{ }^{13} \mathrm{C}$ NMR (100 MHz, $\left.\mathrm{CDCl}_{3}\right): \delta 213.0,167.3,101.7,80.2$, 75.3, 52.2, 32.8, 19.5, 17.9; MS (m/z): $170\left(\mathrm{M}^{+}, 0.17\right), 127$ (100); IR (neat, $\left.\mathrm{cm}^{-1}\right)$ : 3499, 2960, 1964, 1939, 1714, 1258. Elemental analysis: Calcd for $\mathrm{C}_{9} \mathrm{H}_{14} \mathrm{O}_{3}$ : C, 63.51; H, 8.29; Found: C, 63.50; H, 8.29.

(4) 3-(Methoxycarbonyl)-1,2-nonadien-4-ol (3d)

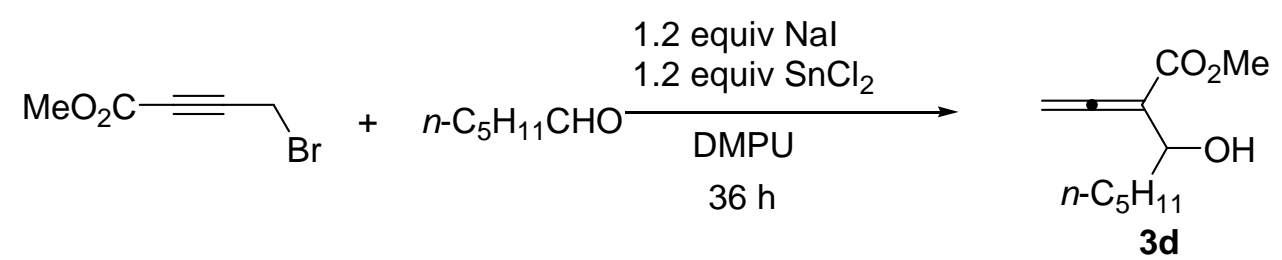

The reaction of $1.7810 \mathrm{~g}$ (10.1 mmol) of 3-(methoxycarbonyl)propargyl bromide, $2.2891 \mathrm{~g}(12.1 \mathrm{mmol})$ of $\mathrm{SnCl}_{2}, 1.8052 \mathrm{~g}(12.0 \mathrm{mmol})$ of $\mathrm{NaI}$, and $0.7958 \mathrm{~g}$ of hexanal $(8.0 \mathrm{mmol})$ in $22 \mathrm{~mL}$ of DMPU afforded $0.9323 \mathrm{~g}$ (59\%) of 3d: Liquid; ${ }^{1} \mathrm{H}$ NMR (400 MHz, $\left.\mathrm{CDCl}_{3}\right) \delta 5.25(\mathrm{~d}, J=1.2 \mathrm{~Hz}, 2 \mathrm{H}), 4.41(\mathrm{t}, J=6.8 \mathrm{~Hz}, 1 \mathrm{H}), 3.76$ (s, 3H), 3.06-2.75 (bs, 1H), 1.66-1.61 (m, 2H), 1.49-1.40 (m, 1H), 1.40-1.20 (m, 5H), $0.87(\mathrm{t}, J=6.4 \mathrm{~Hz}, 3 \mathrm{H}) ;{ }^{13} \mathrm{C}$ NMR $\left(100 \mathrm{MHz}, \mathrm{CDCl}_{3}\right): \delta 212.5,167.5,103.0,80.6$, 69.4, 52.3, 35.3, 31.6, 25.4, 22.6, 14.0; MS (m/z): 198 ( $\left.\mathrm{M}^{+}, 0.10\right), 127$ (100); IR (neat, $\left.\mathrm{cm}^{-1}\right)$ : 3519, 2954, 1964, 1939, 1715, 1259. HRMS calcd for $\mathrm{C}_{11} \mathrm{H}_{18} \mathrm{NaO}_{3}\left(\mathrm{M}^{+}+\mathrm{Na}\right)$ : 221.1148, Found: 221.1145.

(5) 3-(Methoxycarbonyl)-(4-phenyl)-1,2-butadien-4-ol (3e) ${ }^{3}$

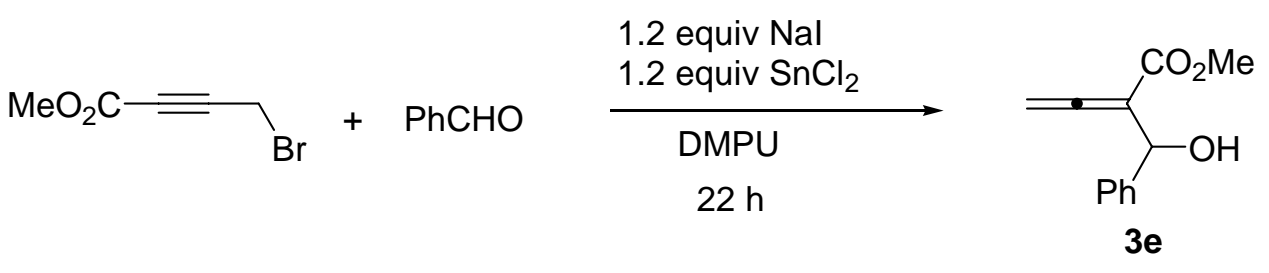


The reaction of $3.5467 \mathrm{~g}$ (20.0 mmol) of 3-(methoxycarbonyl)propargyl bromide, $4.5821 \mathrm{~g}(24.2 \mathrm{mmol})$ of $\mathrm{SnCl}_{2}, 3.6122 \mathrm{~g}(24.1 \mathrm{mmol})$ of $\mathrm{NaI}$, and $1.6899 \mathrm{~g}$ (15.9 mmol) of benzaldehyde in $55 \mathrm{~mL}$ of DMPU afforded $2.5247 \mathrm{~g}$ (78\%) of 3e: Liquid; ${ }^{1} \mathrm{H}$ NMR (400 MHz, $\mathrm{CDCl}_{3}$ ) $\delta$ 7.42-7.26 (m, 5H), 5.59 (s, 1H), 5.25-5.15 (m, 2H), 3.75 (s, 3H), 3.60-3.33 (bs, $1 \mathrm{H}) ;{ }^{13} \mathrm{C}$ NMR (100 MHz, $\left.\mathrm{CDCl}_{3}\right): \delta 213.0,166.8,141.2$, 128.0, 127.5, 126.1, 103.6, 81.0, 71.3, 52.1; MS (m/z): $204\left(\mathrm{M}^{+}, 4.01\right), 203\left(\mathrm{M}^{+}-1\right.$, 22.99), 107 (100); IR (neat, $\mathrm{cm}^{-1}$ ): 3471, 1965, 1713, 1262. HRMS calcd for $\mathrm{C}_{12} \mathrm{H}_{11} \mathrm{O}_{3}$ (M+H): 203.0703, Found: 203.0711.

(6) 3-(Methoxycarbonyl)- 4-(p-tolyl)-1,2-butadien-4-ol (3f)

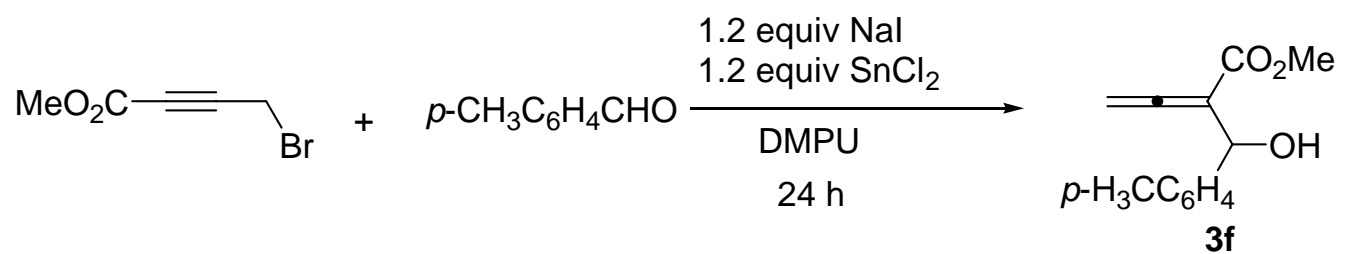

The reaction of $1.7689 \mathrm{~g}$ (10.0 mmol) of 3-(methoxycarbonyl)propargyl bromide, $2.2812 \mathrm{~g}(12.0 \mathrm{mmol})$ of $\mathrm{SnCl}_{2}, 1.7998 \mathrm{~g}(12.0 \mathrm{mmol})$ of $\mathrm{NaI}$, and $0.9500 \mathrm{~g}$ of p-methylbenzaldehyde (7.9 mmol) in $22 \mathrm{~mL}$ of DMPU afforded $1.1740 \mathrm{~g}(68 \%)$ of $3 \mathbf{f}$ : Liquid; ${ }^{1} \mathrm{H}$ NMR (400 MHz, $\left.\mathrm{CDCl}_{3}\right) \delta 7.28(\mathrm{~d}, J=8.0 \mathrm{~Hz}, 2 \mathrm{H}), 7.15$ (d, $J=8.0 \mathrm{~Hz}$, 2H), 5.54 (s, 1H), 5.26-5.17 (m, 2H), 3.74 (s, 3H), 2.34 (s, 3H); ${ }^{13} \mathrm{C}$ NMR (100 MHz, $\left.\mathrm{CDCl}_{3}\right): \delta 213.0,167.1,138.4,137.4,128.9,126.1,103.6,81.1,71.6,52.3,21.1$; MS (m/z): $218\left(\mathrm{M}^{+}, 0.88\right), 217\left(\mathrm{M}^{+}-1,4.39\right), 121$ (100); IR (neat, $\left.\mathrm{cm}^{-1}\right)$ : 3482, 1964, 1931, 1714, 1260. HRMS calcd for $\mathrm{C}_{13} \mathrm{H}_{13} \mathrm{O}_{3}\left(\mathrm{M}^{+}-\mathrm{H}\right)$ : 217.0859, Found: 217.0862.

\section{The Addition-Elimination Reactions of 3-(Methoxycarbonyl)-1,2-allen-4-ols} with MX. 
(1) Preparation of methyl 2-(1'-iodovinyl)pent-2(Z)-enoate (4aa). Typical Procedure.<smiles>C=C(CC)C(O)C(CC)C(C)=O</smiles>

$3 a$

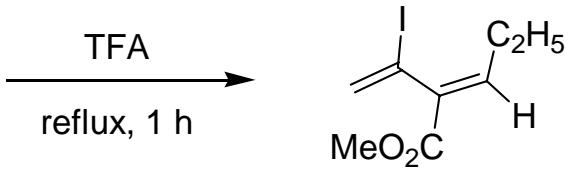

4aa

To a mixture of NaI (151.1 mg, $1.0 \mathrm{mmol})$ and 3a (77.0 mg, $0.49 \mathrm{mmol})$ was added $1.0 \mathrm{~mL}$ of TFA at room temperature. After the addition, the reaction was refluxed at $80{ }^{\circ} \mathrm{C}$ with stirring for $1 \mathrm{~h}$. After the reaction was complete as monitored by TLC, it was cooled to room temperature and slowly quenched by $10 \mathrm{~mL}$ of water followed by neutralization with $\mathrm{NaHCO}_{3}$ until no gas was released. The mixture was extracted with diethyl ether (3 x $25 \mathrm{~mL}$ ) and washed with saturated aqueous solution of $\mathrm{Na}_{2} \mathrm{~S}_{2} \mathrm{O}_{3}$ and brine. The organic layer was dried over anhydrous $\mathrm{Na}_{2} \mathrm{SO}_{4}$. Evaporation and column chromatography on silica gel $\left(30-60{ }^{\circ} \mathrm{C}\right.$ petroleum ether/diethyl ether $=$ 30:1) afforded 4aa (65.4 mg, 50\%): Liquid; ${ }^{1} \mathrm{H}$ NMR (300 MHz, $\left.\mathrm{CDCl}_{3}\right) \delta 6.75$ (t, $J=$ $7.8 \mathrm{~Hz}, 1 \mathrm{H}), 6.08$ (d, $J=1.1 \mathrm{~Hz}, 1 \mathrm{H}), 6.03$ (d, $J=1.1 \mathrm{~Hz}, 1 \mathrm{H}), 3.77$ (s, 3H), 2.29-2.18 (m, 2H), 1.06 (t, $J=7.7 \mathrm{~Hz}, 3 \mathrm{H}) ;{ }^{13} \mathrm{C}$ NMR (75 MHz, $\left.\mathrm{CDCl}_{3}\right): \delta$ 165.3, 146.6, 135.7, 131.2, 96.3, 52.1, 22.8, 12.3; MS (m/z): $267\left(\mathrm{M}^{+}+1,100\right)$; IR (neat, $\left.\mathrm{cm}^{-1}\right)$ : 2962, 1722 , 1603, 1434, 1243. HRMS calcd for $\mathrm{C}_{8} \mathrm{H}_{11} \mathrm{IO}_{2}\left(\mathrm{M}^{+}\right)$: 265.9798, Found: 265.9790 .

(2) Methyl 2-(1'-bromovinyl)pent-2(Z)-enoate (4ab)<smiles>C=C=C(C(C)=O)C(O)CC</smiles>

$3 a$

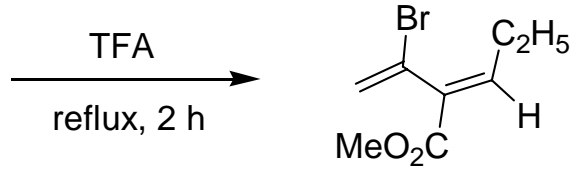

$4 a b$ 
The reaction of $77.1 \mathrm{mg}(0.49 \mathrm{mmol})$ of $\mathbf{3 a}$ and $105.0 \mathrm{mg}(1.0 \mathrm{mmol})$ of $\mathrm{LiBr} \cdot \mathrm{H}_{2} \mathrm{O}$ in $1 \mathrm{~mL}$ of TFA afforded $60.2 \mathrm{mg}$ (56\%) of 4ab: Liquid; ${ }^{1} \mathrm{H}$ NMR (400 $\mathrm{MHz}, \mathrm{CDCl}_{3}$ ) $\delta 6.88(\mathrm{t}, J=7.8 \mathrm{~Hz}, 1 \mathrm{H}), 5.84(\mathrm{~d}, J=2.0 \mathrm{~Hz}, 1 \mathrm{H}), 5.66$ (d, $J=2.0 \mathrm{~Hz}, 1 \mathrm{H}), 3.79$ (s, 3H), 2.32-2.26 (m, 2H), 1.07 (t, $J=7.6 \mathrm{~Hz}, 3 \mathrm{H}) ;{ }^{13} \mathrm{C}$ NMR (100 MHz, $\left.\mathrm{CDCl}_{3}\right): \delta$ 165.5, 148.5, 132.9, 122.6, 122.3, 52.2, 22.9, 12.8; MS (m/z): $220\left(\mathrm{M}^{+}\left({ }^{81} \mathrm{Br}\right), 8.22\right)$, $218\left(\mathrm{M}^{+}\left({ }^{79} \mathrm{Br}\right), 8.32\right), 139$ (100); IR (neat, $\left.\mathrm{cm}^{-1}\right)$ : 2971, 2952, 1724, 1645, 1615, 1267, 1245. HRMS calcd for $\mathrm{C}_{8} \mathrm{H}_{11} \mathrm{BrNaO}_{2}\left(\mathrm{M}^{+}+\mathrm{Na}\right)$ : $242.9822\left({ }^{81} \mathrm{Br}\right), 240.9835\left({ }^{79} \mathrm{Br}\right)$, Found: $242.9811\left({ }^{81} \mathrm{Br}\right), 240.9833\left({ }^{79} \mathrm{Br}\right)$.

(3) Methyl 2-(1'-chlorovinyl)pent-2(Z)-enoate (4ac)<smiles>C=C(C)C(O)C(CC)C(=C)C</smiles>

$3 a$

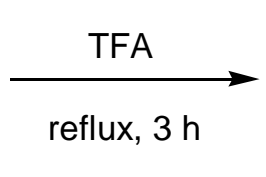

4 equiv

reflux, 3 h<smiles>C=C(Cl)/C(=C\C(C)CC)C(=O)OC</smiles>

$4 a c$

The reaction of $78.8 \mathrm{mg}(0.51 \mathrm{mmol})$ of 3a and $122.1 \mathrm{mg}(2.02 \mathrm{mmol})$ of $\mathrm{LiCl} \cdot \mathrm{H}_{2} \mathrm{O}$ in $1 \mathrm{~mL}$ of TFA afforded $35.5 \mathrm{mg}$ (40\%) of 4ac (There are some unidentified impurities in the ${ }^{1} \mathrm{H}$ NMR spectra. The purity by GC is 92\%) : Liquid; ${ }^{1} \mathrm{H}$ NMR (400 $\left.\mathrm{MHz}, \mathrm{CDCl}_{3}\right) \delta 6.94(\mathrm{t}, J=7.8 \mathrm{~Hz}, 1 \mathrm{H}), 5.62(\mathrm{~d}, J=1.2 \mathrm{~Hz}, 1 \mathrm{H}), 5.26$ (d, $J=1.2 \mathrm{~Hz}$, 1H), 3.79 (s, 3H), 2.36-2.27 (m, 2H), 1.07 (t, $J=7.6 \mathrm{~Hz}, 3 \mathrm{H}) ;{ }^{13} \mathrm{C}$ NMR (100 MHz, $\left.\mathrm{CDCl}_{3}\right): \delta 165.6,149.1,133.0,131.4,118.3,52.2,23.0,13.0 ; \mathrm{MS}(\mathrm{m} / \mathrm{z}): 176\left(\mathrm{M}^{+}\right.$ $\left.\left({ }^{37} \mathrm{Cl}\right), 3.72\right), 174\left(\mathrm{M}^{+}\left({ }^{35} \mathrm{Cl}\right), 11.67\right), 139$ (100); IR (neat, $\left.\mathrm{cm}^{-1}\right): 2971,2955,1725$, 1644, 1619, 1246. HRMS calcd for $\mathrm{C}_{8} \mathrm{H}_{11} \mathrm{ClO}_{2}\left(\mathrm{M}^{+}\right)$: $174.0448\left({ }^{35} \mathrm{Cl}\right)$, Found: $174.0448\left({ }^{35} \mathrm{Cl}\right)$ 
(4) Methyl 2-(1'-iodovinyl)hex-2(Z)-enoate (4ba)

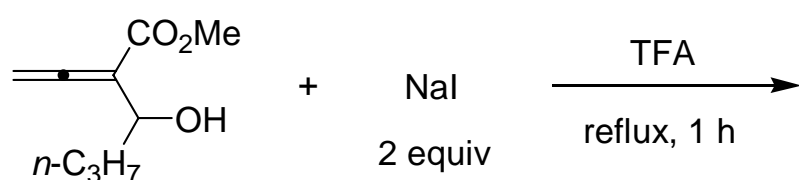

$3 b$<smiles>C=C(I)/C(=C\CCCCCCC)C(=O)OC</smiles>

$4 \mathrm{ba}$

The reaction of $85.2 \mathrm{mg}(0.50 \mathrm{mmol})$ of $3 \mathbf{b}$ and $150.1 \mathrm{mg}(1.0 \mathrm{mmol})$ of $\mathrm{NaI}$ in 1 mL of TFA afforded $102.1 \mathrm{mg}$ (73\%) of $\mathbf{4 b a}$ : Liquid; ${ }^{1} \mathrm{H}$ NMR (300 MHz, $\left.\mathrm{CDCl}_{3}\right) \delta$ 6.75 (t, $J=7.7 \mathrm{~Hz}, 1 \mathrm{H}), 6.07$ (d, $J=1.0 \mathrm{~Hz}, 1 \mathrm{H}), 6.01$ (d, $J=1.0 \mathrm{~Hz}, 1 \mathrm{H}), 3.75$ (s, 3H), 2.18 (q, $J=7.6 \mathrm{~Hz}, 2 \mathrm{H}), 1.56-1.40(\mathrm{~m}, 2 \mathrm{H}), 0.92(\mathrm{t}, J=7.2 \mathrm{~Hz}, 3 \mathrm{H}) ;{ }^{13} \mathrm{C} \mathrm{NMR}$ (75 MHz, $\left.\mathrm{CDCl}_{3}\right): \delta 165.3,145.3,136.2,131.2,96.5,52.1,31.3,21.3,13.9 ; \mathrm{MS}(\mathrm{m} / \mathrm{z})$ : $280\left(\mathrm{M}^{+}, 16.64\right), 93$ (100); IR (neat, $\mathrm{cm}^{-1}$ ): 2959, 1723, 1639, 1605, 1249. HRMS calcd for $\mathrm{C}_{9} \mathrm{H}_{13} \mathrm{IO}_{2}\left(\mathrm{M}^{+}\right)$: 279.9960, Found: 279.9974 .

(5) Methyl 2-(1'-bromovinyl)hex-2(Z)-enoate (4bb)

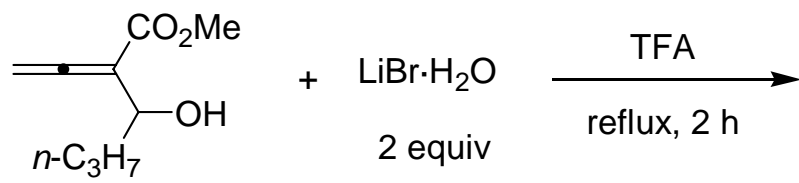

3b<smiles>C=C(Br)/C(=C\CCCCCCCC)C(=O)OC</smiles>

$4 \mathrm{bb}$

The reaction of $83.7 \mathrm{mg}(0.49 \mathrm{mmol})$ of $3 \mathbf{b}$ and $105.1 \mathrm{mg}(1.0 \mathrm{mmol})$ of $\mathrm{LiBr} \cdot \mathrm{H}_{2} \mathrm{O}$ in $1 \mathrm{~mL}$ of TFA afforded $87.2 \mathrm{mg}$ (76\%) of $\mathbf{4 b b}$ : Liquid; ${ }^{1} \mathrm{H}$ NMR (400 MHz, $\mathrm{CDCl}_{3}$ ) $\delta 6.88(\mathrm{t}, J=7.6 \mathrm{~Hz}, 1 \mathrm{H}), 5.83(\mathrm{~d}, J=1.2 \mathrm{~Hz}, 1 \mathrm{H}), 5.64$ (d, $J=1.2 \mathrm{~Hz}, 1 \mathrm{H}), 3.77$ (s, 3H), 2.26 (q, $J=7.6 \mathrm{~Hz}, 2 \mathrm{H}), 1.55-1.43(\mathrm{~m}, 2 \mathrm{H}), 0.94(\mathrm{t}, J=7.4 \mathrm{~Hz}, 3 \mathrm{H}) ;{ }^{13} \mathrm{C} \mathrm{NMR}$ (100 MHz, $\left.\mathrm{CDCl}_{3}\right): \delta 165.3,147.1,133.4,122.6,122.5,52.1,31.4,21.6,13.8 ; \mathrm{MS}$ (m/z): $234\left(\mathrm{M}^{+}\left({ }^{81} \mathrm{Br}\right), 6.15\right), 232\left(\mathrm{M}^{+}\left({ }^{79} \mathrm{Br}\right), 6.45\right), 93$ (100); IR (neat, $\left.\mathrm{cm}^{-1}\right)$ : 2960, 1725, 1614, 1251. HRMS calcd for $\mathrm{C}_{9} \mathrm{H}_{13} \mathrm{BrNaO}_{2}\left(\mathrm{M}^{+}+\mathrm{Na}\right)$ : $256.9979\left({ }^{81} \mathrm{Br}\right)$, 
$254.9991\left({ }^{79} \mathrm{Br}\right)$, Found: $256.9974\left({ }^{81} \mathrm{Br}\right), 254.9994\left({ }^{79} \mathrm{Br}\right)$.

(6) Methyl 2-(1'-chlorovinyl)hex-2(Z)-enoate (4bc)

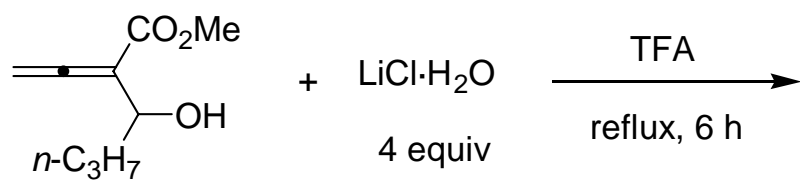

3b<smiles>C=C(Cl)/C(=C\CCCCCCCC)C(=O)OC</smiles>

4bc

The reaction of $84.5 \mathrm{mg}(0.50 \mathrm{mmol})$ of $3 \mathbf{b}$ and $122.0 \mathrm{mg}(2.02 \mathrm{mmol})$ of $\mathrm{LiCl} \cdot \mathrm{H}_{2} \mathrm{O}$ in $1 \mathrm{~mL}$ of TFA afforded $51.1 \mathrm{mg}$ (55\%) of 4bc: Liquid; ${ }^{1} \mathrm{H}$ NMR (400 MHz, $\mathrm{CDCl}_{3}$ ) $\delta 6.94(\mathrm{t}, J=7.8 \mathrm{~Hz}, 1 \mathrm{H}), 5.60(\mathrm{~d}, J=1.2 \mathrm{~Hz}, 1 \mathrm{H}), 5.24$ (d, $J=1.2 \mathrm{~Hz}, 1 \mathrm{H}), 3.77$ (s, 3H), 2.28 (q, $J=7.5 \mathrm{~Hz}, 2 \mathrm{H}), 1.55-1.42(\mathrm{~m}, 2 \mathrm{H}), 0.94(\mathrm{t}, J=7.4 \mathrm{~Hz}, 3 \mathrm{H}) ;{ }^{13} \mathrm{C} \mathrm{NMR}$ (100 MHz, $\left.\mathrm{CDCl}_{3}\right): \delta$ 165.5, 147.7, 133.1, 132.0, 118.3, 52.1, 31.5, 21.7, 13.8; MS (m/z): $190\left(\mathrm{M}^{+}\left({ }^{37} \mathrm{Cl}\right), 3.81\right), 188\left(\mathrm{M}^{+}\left({ }^{35} \mathrm{Cl}\right), 11.30\right), 93$ (100); IR (neat, $\left.\mathrm{cm}^{-1}\right): 2961$, 1727, 1618, 1251. HRMS calcd for $\mathrm{C}_{9} \mathrm{H}_{13} \mathrm{ClNaO}_{2}\left(\mathrm{M}^{+}+\mathrm{Na}\right): 213.0481\left({ }^{37} \mathrm{Cl}\right)$, 211.0496 $\left({ }^{35} \mathrm{Cl}\right)$, Found: $213.0468\left({ }^{37} \mathrm{Cl}\right), 211.0497\left({ }^{35} \mathrm{Cl}\right)$.

(7) Methyl 2-(1'-iodovinyl)-4-methylpent-2(Z)-enoate (4ca)

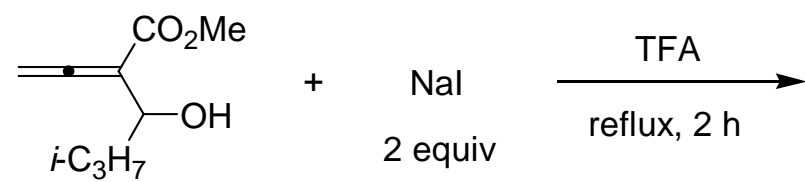

3c<smiles>C=C(I)/C(=C\CCC)C(=O)OC</smiles>

4ca

The reaction of $84.3 \mathrm{mg}(0.50 \mathrm{mmol})$ of $3 \mathrm{c}$ and $150.2 \mathrm{mg}(1.0 \mathrm{mmol})$ of $\mathrm{NaI}$ in 1 mL of TFA afforded 74 mg (53\%) of 4ca: Liquid; ${ }^{1} \mathrm{H}$ NMR (400 MHz, $\left.\mathrm{CDCl}_{3}\right) \delta 6.55$ (d, $J=10.8 \mathrm{~Hz}, 1 \mathrm{H}), 6.07$ (d, $J=0.8 \mathrm{~Hz}, 1 \mathrm{H}), 6.02$ (d, $J=0.8 \mathrm{~Hz}, 1 \mathrm{H}), 3.76(\mathrm{~s}, 3 \mathrm{H})$, 2.82-2.69 (m, 1H), 1.03 (d, $\left.J=6.8 \mathrm{~Hz}, 6 \mathrm{H}) ;{ }^{13} \mathrm{C} \mathrm{NMR} \mathrm{(100} \mathrm{MHz,} \mathrm{CDCl}_{3}\right): \delta$ 165.5, 
151.3, 134.1, 131.0, 96.3, 52.1, 28.8, 21.2; MS (m/z): $280\left(\mathrm{M}^{+}, 19.15\right), 93$ (100); IR (neat, $\mathrm{cm}^{-1}$ ): 2962, 1724, 1643, 1605, 1434, 1248. HRMS calcd for $\mathrm{C}_{9} \mathrm{H}_{13} \mathrm{IO}_{2}\left(\mathrm{M}^{+}\right)$: 279.9955, Found: 279.9957.

(8) Methyl 2-(1'-bromovinyl)-4-methylpent-2(Z)-enoate (4cb)

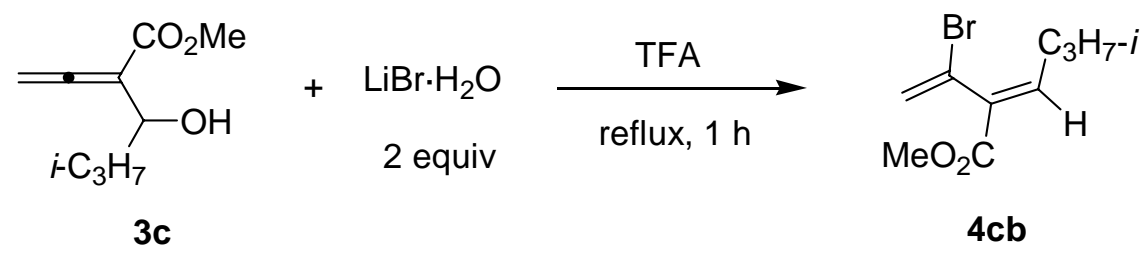

The reaction of $86.2 \mathrm{mg}(0.51 \mathrm{mmol})$ of $3 \mathrm{c}$ and $105.1 \mathrm{mg}(1.0 \mathrm{mmol})$ of $\mathrm{LiBr} \cdot \mathrm{H}_{2} \mathrm{O}$ in $1 \mathrm{~mL}$ of TFA afforded $75.0 \mathrm{mg}(64 \%)$ of $\mathbf{4 c b}$ : Liquid; ${ }^{1} \mathrm{H}$ NMR (400 $\mathrm{MHz}, \mathrm{CDCl}_{3}$ ) $\delta 6.69(\mathrm{~d}, J=11.2 \mathrm{~Hz}, 1 \mathrm{H}), 5.83(\mathrm{~d}, J=1.6 \mathrm{~Hz}, 1 \mathrm{H}), 5.66$ (d, $J=1.6 \mathrm{~Hz}, 1 \mathrm{H}), 3.79$ (s, 3H), 2.86-2.74 (m, 1H), 1.05 (d, $J=6.4 \mathrm{~Hz}, 6 \mathrm{H}) ;{ }^{13} \mathrm{C}$ NMR (100 MHz, $\left.\mathrm{CDCl}_{3}\right): \delta$ 165.6, 153.2, 131.1, 122.5, 122.4, 52.2, 28.9, 21.7; MS (m/z): $234\left(\mathrm{M}^{+}\left({ }^{81} \mathrm{Br}\right), 0.98\right)$, $232\left(\mathrm{M}^{+}\left({ }^{79} \mathrm{Br}\right), 1.01\right), 153$ (100); IR (neat, $\left.\mathrm{cm}^{-1}\right)$ : 2964, 1725, 1614, 1249. HRMS calcd for $\mathrm{C}_{9} \mathrm{H}_{13} \mathrm{BrO}_{2}\left(\mathrm{M}^{+}\right)$: $234.0082\left({ }^{81} \mathrm{Br}\right), 232.0093\left({ }^{79} \mathrm{Br}\right)$, Found: $234.0069\left({ }^{81} \mathrm{Br}\right)$, $232.0098\left({ }^{79} \mathrm{Br}\right)$.

(9) Methyl 2-(1'-chlorovinyl)-4-methylpent-2(Z)-enoate (4cc)

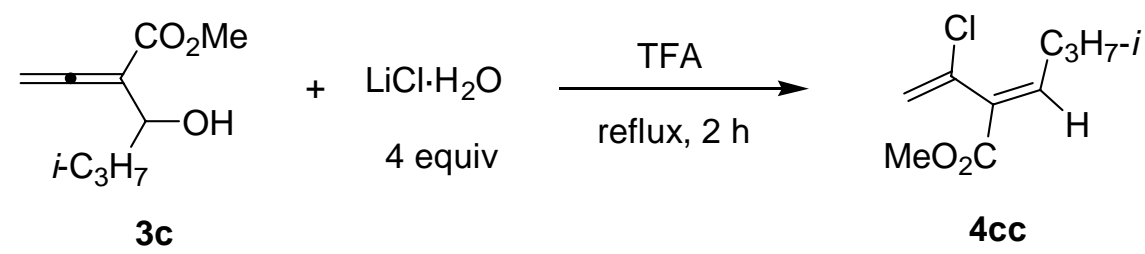

The reaction of $85.7 \mathrm{mg}(0.50 \mathrm{mmol})$ of $3 \mathrm{c}$ and $122.1 \mathrm{mg}(2.01 \mathrm{mmol})$ of $\mathrm{LiCl} \cdot \mathrm{H}_{2} \mathrm{O}$ in $1 \mathrm{~mL}$ of TFA afforded $36.9 \mathrm{mg}$ (39\%) of 4cc: Liquid; ${ }^{1} \mathrm{H}$ NMR (400 MHz, $\mathrm{CDCl}_{3}$ ) 
$\delta 6.73(\mathrm{~d}, J=10.8 \mathrm{~Hz}, 1 \mathrm{H}), 5.59(\mathrm{~d}, J=1.0 \mathrm{~Hz}, 1 \mathrm{H}), 5.25$ (d, $J=1.0 \mathrm{~Hz}, 1 \mathrm{H}), 3.77$ (s, 3H), 2.85-2.73 (m, 1H), 1.04 (d, $J=6.8 \mathrm{~Hz}, 6 \mathrm{H}) ;{ }^{13} \mathrm{C}$ NMR (100 MHz, $\left.\mathrm{CDCl}_{3}\right): \delta$ 165.7, 153.7, 133.2, 129.6, 118.0, 52.2, 28.9, 21.8; MS (m/z): $190\left(\mathrm{M}^{+}\left({ }^{37} \mathrm{Cl}\right), 4.81\right)$, $188\left(\mathrm{M}^{+}\left({ }^{35} \mathrm{Cl}\right), 14.93\right), 93$ (100); IR (neat, $\left.\mathrm{cm}^{-1}\right): 2964,1726,1618,1251$. HRMS calcd for $\mathrm{C}_{9} \mathrm{H}_{13} \mathrm{ClO}_{2}\left(\mathrm{M}^{+}\right)$: $190.0583\left({ }^{37} \mathrm{Cl}\right), 188.0600\left({ }^{35} \mathrm{Cl}\right)$, Found: $190.0574\left({ }^{37} \mathrm{Cl}\right)$, $188.0604\left({ }^{35} \mathrm{Cl}\right)$

(10) Methyl 2-(1'-iodovinyl)oct-2(Z)-enoate (4da)

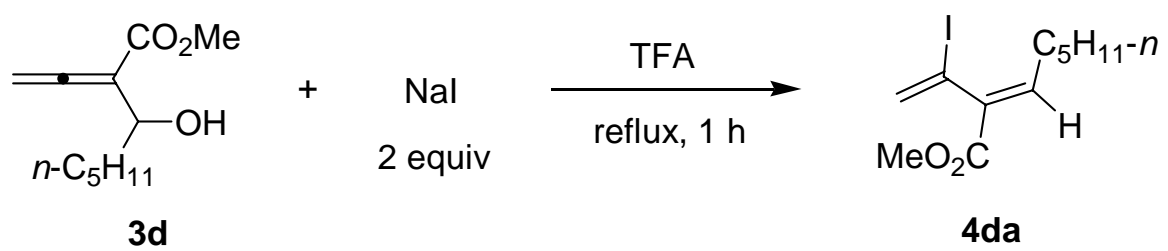

The reaction of $96.0 \mathrm{mg}(0.48 \mathrm{mmol})$ of $\mathbf{3 d}$ and $150.1 \mathrm{mg}(1.0 \mathrm{mmol})$ of $\mathrm{NaI}$ in 1 $\mathrm{mL}$ of TFA afforded $111.7 \mathrm{mg}$ (75\%) of 4da: Liquid; ${ }^{1} \mathrm{H}$ NMR (400 MHz, $\left.\mathrm{CDCl}_{3}\right) \delta$ 6.77 (t, $J=7.6 \mathrm{~Hz}, 1 \mathrm{H}), 6.09$ (d, $J=1.2 \mathrm{~Hz}, 1 \mathrm{H}), 6.02$ (d, $J=1.2 \mathrm{~Hz}, 1 \mathrm{H}), 3.77$ (s, 3H), 2.21 (q, $J=7.6 \mathrm{~Hz}, 2 \mathrm{H}), 1.51-1.38$ (m, 2H), 1.36-1.26 (m, 4H), 0.88 (t, $J=7.0$ $\mathrm{Hz}, 3 \mathrm{H}) ;{ }^{13} \mathrm{C} \mathrm{NMR}\left(100 \mathrm{MHz}, \mathrm{CDCl}_{3}\right): \delta$ 165.3, 145.6, 136.1, 131.2, 96.6, 52.1, 31.5, 29.4, 27.7, 22.4, 13.9; MS (m/z): $308\left(\mathrm{M}^{+}, 16.31\right), 121$ (100); IR (neat, $\left.\mathrm{cm}^{-1}\right)$ : 2955, 2927, 1724, 1640, 1606, 1434, 1251, 1051. HRMS calcd for $\mathrm{C}_{11} \mathrm{H}_{17} \mathrm{INaO}_{2}\left(\mathrm{M}^{+}+\mathrm{Na}\right)$ : 331.0165, Found: 331.0161.

(11) Methyl 2-(1'-bromovinyl)oct-2(Z)-enoate (4db) 


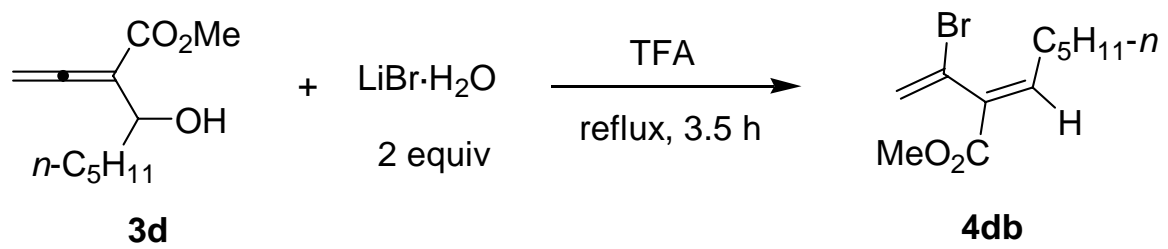

The reaction of $98.1 \mathrm{mg}(0.49 \mathrm{mmol})$ of $\mathbf{3 d}$ and $104.5 \mathrm{mg}(1.0 \mathrm{mmol})$ of $\mathrm{LiBr} \cdot \mathrm{H}_{2} \mathrm{O}$ in $1 \mathrm{~mL}$ of TFA afforded $86.4 \mathrm{mg}(67 \%)$ of $\mathbf{4 d b}$ : Liquid; ${ }^{1} \mathrm{H}$ NMR (400 MHz, $\mathrm{CDCl}_{3}$ ) $\delta 6.90(\mathrm{t}, J=7.6 \mathrm{~Hz}, 1 \mathrm{H}), 5.84(\mathrm{~d}, J=1.6 \mathrm{~Hz}, 1 \mathrm{H}), 5.65$ (d, $J=1.6 \mathrm{~Hz}, 1 \mathrm{H}), 3.78$ (s, 3H), 2.28 (q, $J=7.6 \mathrm{~Hz}, 2 \mathrm{H}), 1.51-1.39(\mathrm{~m}, 2 \mathrm{H}), 1.35-1.26(\mathrm{~m}, 4 \mathrm{H}), 0.88(\mathrm{t}, J=6.8$ $\mathrm{Hz}, 3 \mathrm{H}) ;{ }^{13} \mathrm{C} \mathrm{NMR}\left(100 \mathrm{MHz}, \mathrm{CDCl}_{3}\right): \delta$ 165.4, 147.5, 133.1, 122.7, 122.4, 52.2, 31.4, 29.4, 27.9, 22.4, 13.9; MS (m/z): 262 ( $\left.\mathrm{M}^{+}\left({ }^{81} \mathrm{Br}\right), 1.01\right), 260\left(\mathrm{M}^{+}\left({ }^{79} \mathrm{Br}\right), 1.03\right), 181$ (100); IR (neat, $\mathrm{cm}^{-1}$ ): 2955, 2928, 1726, 1647, 1615, 1457, 1435, 1254. HRMS calcd for $\mathrm{C}_{11} \mathrm{H}_{17} \mathrm{O}_{2}\left(\mathrm{M}^{+}-\mathrm{Br}\right)$ : 181.1223. Found: 181.1223. HRMS calcd for $\mathrm{C}_{10} \mathrm{H}_{14} \mathrm{BrO}$ $\left(\mathrm{M}^{+}-\mathrm{OCH}_{3}\right)$ : $231.0211\left({ }^{81} \mathrm{Br}\right), 229.0223\left({ }^{79} \mathrm{Br}\right)$, Found: $231.0202\left({ }^{81} \mathrm{Br}\right), 229.0220$ $\left({ }^{79} \mathrm{Br}\right)$.

(12) Methyl 2-(1'-chlorovinyl)oct-2(Z)-enoate (4dc)

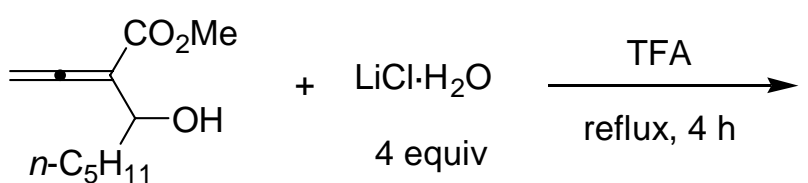

3d<smiles>C=C(Cl)/C(=C\CCCCCCC)C(C)Cl</smiles>

4dc

The reaction of $98.6 \mathrm{mg}(0.50 \mathrm{mmol})$ of $\mathbf{3 d}$ and $118.2 \mathrm{mg}(1.95 \mathrm{mmol})$ of $\mathrm{LiCl} \cdot \mathrm{H}_{2} \mathrm{O}$ in $1 \mathrm{~mL}$ of TFA afforded $50.8 \mathrm{mg}$ (47\%) of 4dc: Liquid; ${ }^{1} \mathrm{H}$ NMR (400 $\mathrm{MHz}, \mathrm{CDCl}_{3}$ ) $\delta 6.94(\mathrm{t}, J=8.0 \mathrm{~Hz}, 1 \mathrm{H}), 5.60$ (d, $J=1.2 \mathrm{~Hz}, 1 \mathrm{H}), 5.24$ (d, $J=1.2 \mathrm{~Hz}, 1 \mathrm{H}), 3.77$ (s, 3H), 2.28 (q, $J=8.0 \mathrm{~Hz}, 2 \mathrm{H}), 1.51-1.38(\mathrm{~m}, 2 \mathrm{H}), 1.35-1.22(\mathrm{~m}, 4 \mathrm{H}), 0.87$ (t, $J=7.0$ $\mathrm{Hz}, 3 \mathrm{H}) ;{ }^{13} \mathrm{C} \mathrm{NMR}\left(100 \mathrm{MHz}, \mathrm{CDCl}_{3}\right): \delta 165.5,148.1,133.0,131.7,118.3,52.2,31.4$, 
29.5, 28.1, 22.4, 13.9; MS (m/z): $218\left(\mathrm{M}^{+}\left({ }^{37} \mathrm{Cl}\right), 0.93\right), 216\left(\mathrm{M}^{+}\left({ }^{35} \mathrm{Cl}\right), 2.82\right), 181$ (100); IR (neat, $\mathrm{cm}^{-1}$ ): 2956, 2929, 1727, 1650, 1619, 1458, 1435, 1255, 1053. HRMS calcd for $\mathrm{C}_{11} \mathrm{H}_{17} \mathrm{ClO}_{2}\left(\mathrm{M}^{+}\right)$: $218.0899\left({ }^{37} \mathrm{Cl}\right), 216.0912\left({ }^{35} \mathrm{Cl}\right)$, Found: $218.0886\left({ }^{37} \mathrm{Cl}\right)$, $216.0917\left({ }^{35} \mathrm{Cl}\right)$.

(13) Methyl 2-(1'-iodovinyl)-3-phenylprop-2(Z)-enoate (4ea)

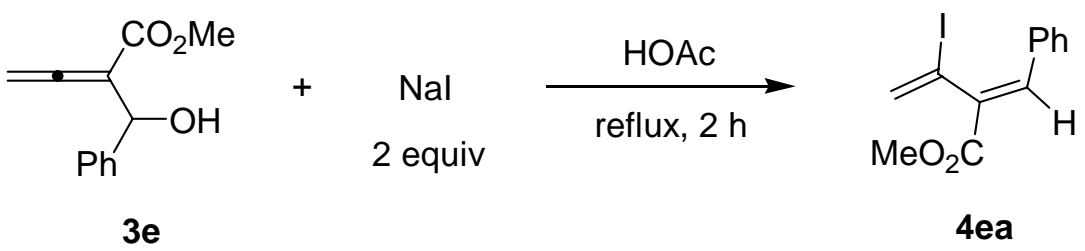

The reaction of $100.4 \mathrm{mg}(0.49 \mathrm{mmol})$ of $3 \mathbf{e}$ and $150.2 \mathrm{mg}(1.00 \mathrm{mmol})$ of $\mathrm{NaI}$ in 1 mL of HOAc afforded $61.6 \mathrm{mg}$ (40\%) of 4ea (There are some unidentified impurities in the ${ }^{1} \mathrm{H}$ NMR spectra. The purity by GC is 92\%): Liquid; ${ }^{1} \mathrm{H}$ NMR (400 MHz, $\left.\mathrm{CDCl}_{3}\right) \delta$ 7.76-7.65 (m, 2H), 7.52 (s, 1H), 7.45-7.38 (m, 3H), $6.21(\mathrm{~d}, J=1.4 \mathrm{~Hz}, 1 \mathrm{H})$, $6.13(\mathrm{~d}, J=1.4 \mathrm{~Hz}, 1 \mathrm{H}), 3.86(\mathrm{~s}, 3 \mathrm{H}) ;{ }^{13} \mathrm{C} \mathrm{NMR}\left(100 \mathrm{MHz}, \mathrm{CDCl}_{3}\right): \delta 166.0,139.6$, 133.9, 133.5, 131.8, 131.1, 130.0, 128.6, 98.4, 52.6; MS (m/z): $314\left(\mathrm{M}^{+}, 8.26\right), 128$ (100); IR (neat, $\mathrm{cm}^{-1}$ ): 2949, 1716, 1635, 1597, 1448, 1434, 1255, 1201. HRMS calcd for $\mathrm{C}_{12} \mathrm{H}_{11} \mathrm{IO}_{2}\left(\mathrm{M}^{+}\right)$: 313.9798, Found: 313.9801.

(14) Methyl 2-(1'-bromovinyl)-3-phenylprop-2(Z)-enoate (4eb)

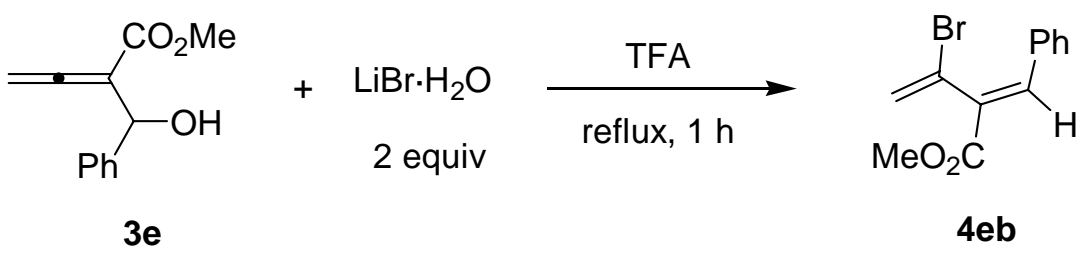

The reaction of $101.5 \mathrm{mg}(0.50 \mathrm{mmol})$ of $3 \mathbf{e}$ and $105.0 \mathrm{mg}(1.0 \mathrm{mmol})$ of $\mathrm{LiBr} \cdot \mathrm{H}_{2} \mathrm{O}$ 
in $1 \mathrm{~mL}$ of TFA afforded 4eb (58.6 mg, 44\%).

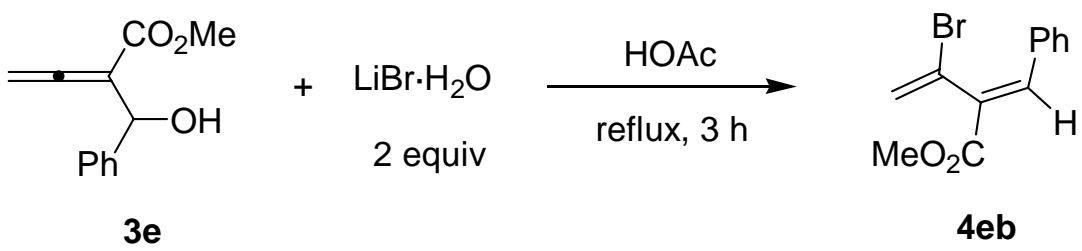

The reaction of $103.7 \mathrm{mg}(0.51 \mathrm{mmol})$ of $3 \mathbf{e}$ and $105.0 \mathrm{mg}(1.0 \mathrm{mmol})$ of $\mathrm{LiBr} \cdot \mathrm{H}_{2} \mathrm{O}$ in $1 \mathrm{~mL}$ of HOAc afforded 4eb (59.1 mg, 44\%).

4eb: Liquid; ${ }^{1} \mathrm{H}$ NMR (400 MHz, $\left.\mathrm{CDCl}_{3}\right) \delta$ 7.74-7.66 (m, 2H), 7.65 (s, 1H), 7.44-7.35 (m, 3H), 5.86 (d, $J=1.8 \mathrm{~Hz}, 1 \mathrm{H}), 5.79$ (d, $J=1.8 \mathrm{~Hz}, 1 \mathrm{H}), 3.86(\mathrm{~s}, 3 \mathrm{H}) ;{ }^{13} \mathrm{C}$ NMR (100 MHz, $\left.\mathrm{CDCl}_{3}\right): \delta$ 166.0, 141.5, 133.3, 130.8, 130.1, 128.5, 123.6, 123.2, 52.5; MS (m/z): $268\left(\mathrm{M}^{+}\left({ }^{81} \mathrm{Br}\right), 4.34\right), 266\left(\mathrm{M}^{+}\left({ }^{79} \mathrm{Br}\right), 4.40\right), 128$ (100); IR (neat, $\mathrm{cm}^{-1}$ ): 2950, 1717, 1644, 1604, 1448, 1434, 1256, 1202. HRMS calcd for $\mathrm{C}_{12} \mathrm{H}_{11} \mathrm{BrO}_{2}$ $\left(\mathrm{M}^{+}\right)$: $267.9927\left({ }^{81} \mathrm{Br}\right), 265.9937\left({ }^{79} \mathrm{Br}\right)$, Found: $267.9919\left({ }^{81} \mathrm{Br}\right), 265.9939\left({ }^{79} \mathrm{Br}\right)$.

(15) Methyl 2-(1'-chlorovinyl)-3-phenylprop-2(Z)-enoate (4ec)

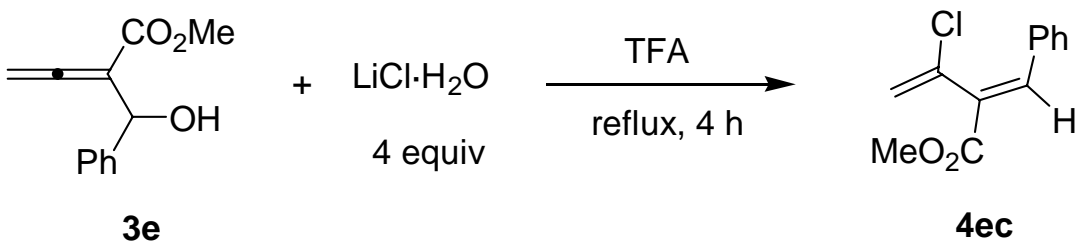

The reaction of $103.0 \mathrm{mg}(0.50 \mathrm{mmol})$ of $3 \mathbf{e}$ and $120.8 \mathrm{mg}(2.00 \mathrm{mmol})$ of $\mathrm{LiCl} \cdot \mathrm{H}_{2} \mathrm{O}$ in $1 \mathrm{~mL}$ of TFA afforded 4 ec (29.3 mg, 26\%).

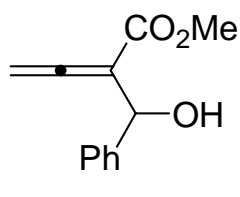

$3 e$<smiles>C=C(Cl)/C(=C\c1ccccc1)C(C)(C)C</smiles>

The reaction of $103.3 \mathrm{mg}(0.51 \mathrm{mmol})$ of $3 \mathbf{e}$ and $123.1 \mathrm{mg}(2.03 \mathrm{mmol})$ of $\mathrm{LiCl} \cdot \mathrm{H}_{2} \mathrm{O}$ in $1 \mathrm{~mL}$ of HOAc afforded $\mathbf{4 e c}$ (33.9 mg, 31\%). 
4ec: Liquid; ${ }^{1} \mathrm{H}$ NMR (400 MHz, $\left.\mathrm{CDCl}_{3}\right) \delta 7.71$ (s, 1H), 7.69-7.61 (m, 2H), 7.40-7.35 (m, 3H), 5.61 (d, $J=1.8 \mathrm{~Hz}, 1 \mathrm{H}), 5.37$ (d, $J=1.8 \mathrm{~Hz}, 1 \mathrm{H}), 3.86$ (s, 3H); ${ }^{13} \mathrm{C}$ NMR (100 MHz, $\left.\mathrm{CDCl}_{3}\right): \delta$ 166.1, 142.3, 134.0, 133.4, 130.5, 130.1, 129.4, 128.6, 118.9, 52.6; MS (m/z): $224\left(\mathrm{M}^{+}\left({ }^{37} \mathrm{Cl}\right), 3.78\right), 222\left(\mathrm{M}^{+}\left({ }^{35} \mathrm{Cl}\right), 11.39\right), 128$ (100); IR (neat, $\mathrm{cm}^{-1}$ ): 2951, 1717, 1649, 1608, 1448, 1435, 1258, 1202. HRMS calcd for $\mathrm{C}_{12} \mathrm{H}_{11} \mathrm{ClO}_{2}\left(\mathrm{M}^{+}\right): 224.0430\left({ }^{37} \mathrm{Cl}\right), 222.0442\left({ }^{35} \mathrm{Cl}\right)$, Found: $224.0417\left({ }^{37} \mathrm{Cl}\right), 222.0446$ $\left({ }^{35} \mathrm{Cl}\right)$.

(16) Methyl 2-(1'-iodovinyl)-3-(p-tolyl)prop-2(Z)-enoate (4fa)

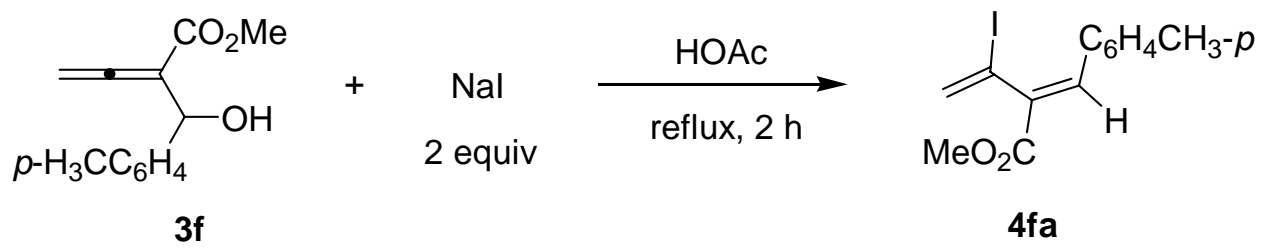

The reaction of $107.7 \mathrm{mg}(0.49 \mathrm{mmol})$ of $\mathbf{3 f}$ and $150.2 \mathrm{mg}(1.00 \mathrm{mmol})$ of $\mathrm{NaI}$ in 1 mL of HOAc afforded $117.5 \mathrm{mg}$ (73\%) of $\mathbf{4 f a}$ (There are some unidentified impurities in the ${ }^{1} \mathrm{H}$ NMR spectra. The purity by GC is $95.5 \%$ ): Liquid; ${ }^{1} \mathrm{H}$ NMR (400 MHz, $\left.\mathrm{CDCl}_{3}\right) \delta 7.62(\mathrm{~d}, J=7.6 \mathrm{~Hz}, 2 \mathrm{H}), 7.49(\mathrm{~s}, 1 \mathrm{H}), 7.20$ (d, $\left.J=8.0 \mathrm{~Hz}, 2 \mathrm{H}\right), 6.20$ (d, $J=$ $1.6 \mathrm{~Hz}, 1 \mathrm{H}), 6.13$ (d, $J=1.6 \mathrm{~Hz}, 1 \mathrm{H}), 3.85$ (s, 3H), 2.37 (s, 3H); ${ }^{13} \mathrm{C}$ NMR (100 MHz, $\left.\mathrm{CDCl}_{3}\right): \delta 166.0,140.5,139.6,132.8,131.6,131.3,130.6,129.3,98.9,52.4,21.5 ; \mathrm{MS}$ (m/z): $328\left(\mathrm{M}^{+}, 9.21\right), 142$ (100); IR (neat, $\left.\mathrm{cm}^{-1}\right)$ : 2949, 1716, 1659, 1605, 1434, 1256. HRMS calcd for $\mathrm{C}_{13} \mathrm{H}_{13} \mathrm{IO}_{2}\left(\mathrm{M}^{+}\right)$: 327.9955, Found: 327.9955. 


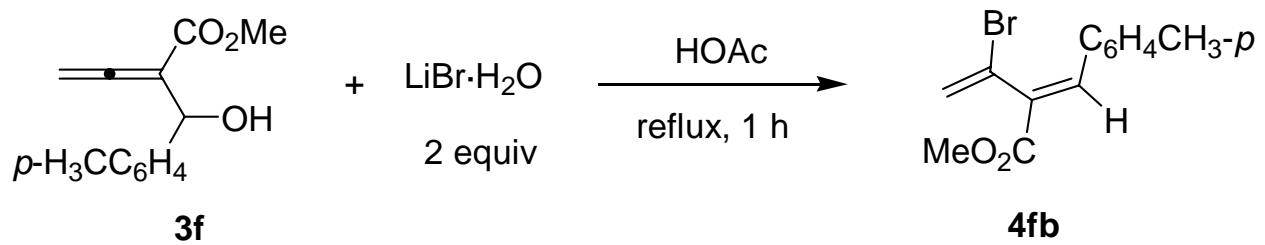

The reaction of $111.8 \mathrm{mg}(0.51 \mathrm{mmol})$ of $\mathbf{3 f}$ and $105.1 \mathrm{mg}(1.0 \mathrm{mmol})$ of $\mathrm{LiBr} \cdot \mathrm{H}_{2} \mathrm{O}$ in $1 \mathrm{~mL}$ of HOAc afforded $88.8 \mathrm{mg}(62 \%)$ of $\mathbf{4 f b}$ : Liquid; ${ }^{1} \mathrm{H}$ NMR (400 MHz, $\left.\mathrm{CDCl}_{3}\right)$ $\delta 7.62(\mathrm{~s}, 1 \mathrm{H}), 7.61$ (d, $J=8.4 \mathrm{~Hz}, 2 \mathrm{H}), 7.20$ (d, $J=8.4 \mathrm{~Hz}, 2 \mathrm{H}), 5.86$ (d, $J=1.6 \mathrm{~Hz}$, 1H), 5.80 (d, $J=1.6 \mathrm{~Hz}, 1 \mathrm{H}), 3.85$ (s, 3H), 2.37 (s, 3H); ${ }^{13} \mathrm{C}$ NMR (100 MHz, $\left.\mathrm{CDCl}_{3}\right): \delta 166.1,141.5,140.7,130.9,130.5,129.7,129.3,124.0,123.0,52.4,21.4$ MS (m/z): $282\left(\mathrm{M}^{+}\left({ }^{81} \mathrm{Br}\right), 4.46\right), 280\left(\mathrm{M}^{+}\left({ }^{79} \mathrm{Br}\right), 4.51\right), 142$ (100); IR (neat, $\left.\mathrm{cm}^{-1}\right)$ : 2950, 1716, 1640, 1604, 1511, 1434, 1258, 1050. HRMS calcd for $\mathrm{C}_{13} \mathrm{H}_{13} \mathrm{BrO}_{2}\left(\mathrm{M}^{+}\right)$: 282.0084 ( $\left.{ }^{81} \mathrm{Br}\right), 280.0093\left({ }^{79} \mathrm{Br}\right)$, Found: $282.0071\left({ }^{81} \mathrm{Br}\right), 280.0096\left({ }^{79} \mathrm{Br}\right)$.

(18) Methyl 2-(1'-chlorovinyl)-3-(p-tolyl)prop-2(Z)-enoate (4fc)

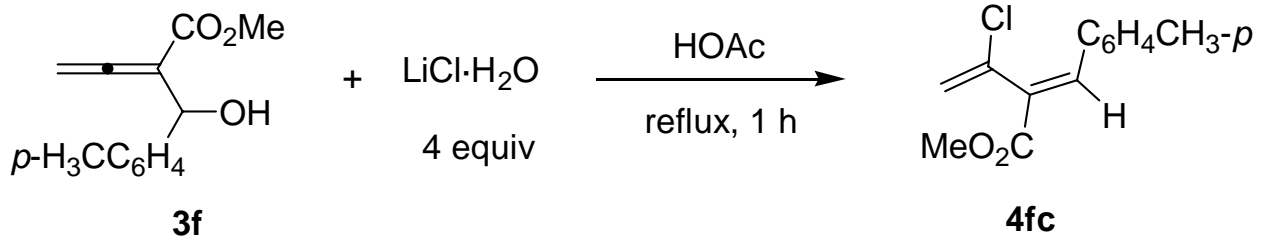

The reaction of $110.5 \mathrm{mg}(0.51 \mathrm{mmol})$ of $\mathbf{3 f}$ and $120.5 \mathrm{mg}(1.99 \mathrm{mmol})$ of $\mathrm{LiCl} \cdot \mathrm{H}_{2} \mathrm{O}$ in $1 \mathrm{~mL}$ of $\mathrm{HOAc}$ afforded $70.4 \mathrm{mg}$ (59\%) of $\mathbf{4 f c}$ : Liquid; ${ }^{1} \mathrm{H}$ NMR (400 $\left.\mathrm{MHz}, \mathrm{CDCl}_{3}\right) \delta 7.69(\mathrm{~s}, 1 \mathrm{H}), 7.57(\mathrm{~d}, J=8.4 \mathrm{~Hz}, 2 \mathrm{H}), 7.19(\mathrm{~d}, J=8.4 \mathrm{~Hz}, 2 \mathrm{H}), 5.62$ (d, $J=1.4 \mathrm{~Hz}, 1 \mathrm{H}), 5.38$ (d, $J=1.4 \mathrm{~Hz}, 1 \mathrm{H}), 3.85$ (s, 3H), 2.37 (s, 3H); ${ }^{13} \mathrm{C}$ NMR (100 MHz, $\left.\mathrm{CDCl}_{3}\right): \delta 166.2,142.3,140.7,134.2,130.6,130.5,129.3,128.2,118.8$, 52.4, 21.4; MS (m/z): $238\left(\mathrm{M}^{+}\left({ }^{37} \mathrm{Cl}\right), 3.48\right), 236\left(\mathrm{M}^{+}\left({ }^{35} \mathrm{Cl}\right), 10.33\right), 142$ (100); IR (neat, $\mathrm{cm}^{-1}$ ): 2951, 1716, 1641, 1607, 1512, 1435, 1258, 1052. HRMS calcd for 
$\mathrm{C}_{13} \mathrm{H}_{13} \mathrm{ClO}_{2}\left(\mathrm{M}^{+}\right)$: $238.0588\left({ }^{37} \mathrm{Cl}\right), 236.0599\left({ }^{35} \mathrm{Cl}\right)$, Found: $238.0574\left({ }^{37} \mathrm{Cl}\right), 236.0600$ $\left({ }^{35} \mathrm{Cl}\right)$.

3. Reduction of methyl 2-(1'-iodovinyl)hex-2(Z)-enoate (4ba)

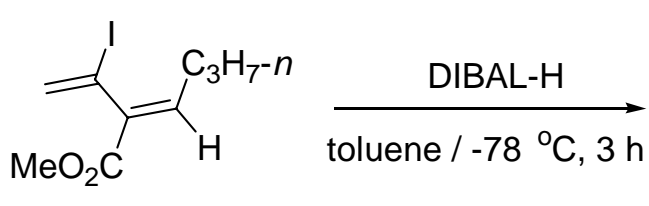

$4 \mathrm{ba}$

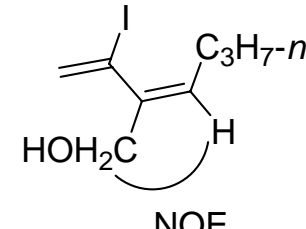

$5 \mathrm{ba}$

DIBAL-H (1.4 mL, $1 \mathrm{M}$ in toluene, $1.4 \mathrm{mmol})$ was added dropwise to a solution of 4ba (180.0 mg, $0.64 \mathrm{mmol})$ in toluene $(3 \mathrm{~mL})$ at $-78{ }^{\circ} \mathrm{C}$. After complete conversion of the starting materials as monitored by TLC, the reaction mixture was quenched with 2 $\mathrm{mL}$ of $\mathrm{CH}_{3} \mathrm{OH}$ and $5 \mathrm{~mL}$ of water. The organic layer was separated, and the aqueous layer was extracted with diethyl ether (3 x $25 \mathrm{~mL}$ ). The combined organic layer was dried over $\mathrm{Na}_{2} \mathrm{SO}_{4}$. Evaporation and column chromatography on silica gel (petroleum ether/ethyl acetate $=10: 1)$ afforded $118.7 \mathrm{mg}(73 \%)$ of 5ba: Liquid; ${ }^{1} \mathrm{H}$ NMR (400 $\left.\mathrm{MHz}, \mathrm{CDCl}_{3}\right) \delta 6.03(\mathrm{~d}, J=1.2 \mathrm{~Hz}, 1 \mathrm{H}), 5.99(\mathrm{~d}, J=1.2 \mathrm{~Hz}, 1 \mathrm{H}), 5.53(\mathrm{t}, J=7.4 \mathrm{~Hz}$, 1H), 4.15 (s, 2H), 2.13-2.00 (m, 3H), 1.45-1.34 (m, 2H), $0.90(\mathrm{t}, J=7.2 \mathrm{~Hz}, 3 \mathrm{H}) ;{ }^{13} \mathrm{C}$ NMR (100 MHz, $\left.\mathrm{CDCl}_{3}\right): \delta 142.4,129.9,129.8,102.5,64.9,30.7,22.0,13.8 ; \mathrm{MS}$ (m/z): $252\left(\mathrm{M}^{+}, 4.26\right), 79$ (100); IR (neat, $\left.\mathrm{cm}^{-1}\right)$ : 3333, 2958, 2929, 2870, 1608, 1456 , 1107, 900. HRMS calcd for $\mathrm{C}_{8} \mathrm{H}_{13} \mathrm{IO}\left(\mathrm{M}^{+}\right)$: 274.9903, Found: 274.9914 .

\section{Synthetic application of methyl 2-(1'-iodovinyl)hex-2(Z)-enoate (4ba)}

(1) Methyl 2-(4'-phenylbut-1'-en-3'-yn-2'-yl)hex-2(E)-enoate (6ba) 


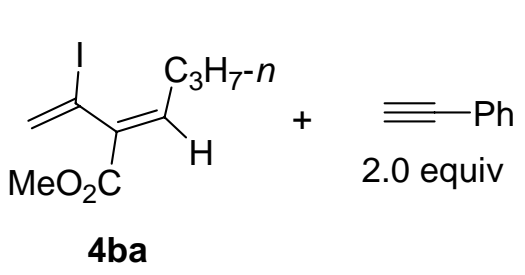

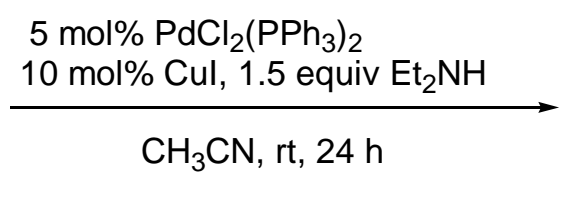

$\mathrm{CH}_{3} \mathrm{CN}, \mathrm{rt}, 24 \mathrm{~h}$<smiles>C=C(C#Cc1ccccc1)/C(=C\CCCCCO)COC</smiles>

6ba

A mixture of $\mathrm{Pd}\left(\mathrm{PPh}_{3}\right)_{2} \mathrm{Cl}_{2}$ (6.8 mg, $\left.5 \mathrm{~mol} \%, 0.01 \mathrm{mmol}\right)$, $\mathrm{CuI}$ (4.1 mg, $10 \mathrm{~mol} \%$, $0.02 \mathrm{mmol}), \mathrm{Et}_{2} \mathrm{NH}$ (22.2 mg, $\left.0.30 \mathrm{mmol}\right)$, phenylacetylene (41.6 mg, $0.41 \mathrm{mmol}$ ), and 4 ba (55.9 mg, $0.20 \mathrm{mmol}$ ) in $\mathrm{CH}_{3} \mathrm{CN}(2 \mathrm{~mL}$ ) was stirred at room temperature over a period of $24 \mathrm{~h}$ under nitrogen. After complete conversion of the starting materials as monitored by TLC, the reaction mixture was diluted with $10 \mathrm{~mL}^{\text {of }} \mathrm{Et}_{2} \mathrm{O}$ and quenched with $5 \mathrm{~mL}$ of water. The organic layer was separated, and the aqueous layer was extracted with diethyl ether ( 3 x $25 \mathrm{~mL}$ ). The combined organic layer was dried over $\mathrm{Na}_{2} \mathrm{SO}_{4}$. Evaporation and column chromatography on silica gel (petroleum ether/diethyl ether = 30:1) afforded 6ba (44.1 mg, 87\%): Liquid; ${ }^{1} \mathrm{H}$ NMR (400 MHz, $\left.\mathrm{CDCl}_{3}\right) \delta$ 7.46-7.38 (m, 2H), 7.34-7.26 (m, 3H), $6.94(\mathrm{t}, J=7.6 \mathrm{~Hz}, 1 \mathrm{H}), 5.85(\mathrm{~s}, 1 \mathrm{H})$, 5.39 (s, 1H), 3.79 (s, 3H), 2.32 (q, $J=7.3 \mathrm{~Hz}, 2 \mathrm{H}), 1.59-1.45$ (m, 2H), 0.96 (t, $J=7.2$ $\mathrm{Hz}, 3 \mathrm{H}) ;{ }^{13} \mathrm{C}$ NMR (100 MHz, $\left.\mathrm{CDCl}_{3}\right): \delta 166.6,145.8,131.8,131.6,128.23,128.18$, 126.7, 125.1, 123.0, 89.1, 88.7, 52.0, 31.4, 21.9, 13.8; MS (m/z): 254 (M+1 62.02), 165 (100); IR (neat, $\mathrm{cm}^{-1}$ ): 2959, 1720, 1639, 1600, 1489, 1435, 1250, 1058. HRMS calcd for $\mathrm{C}_{17} \mathrm{H}_{18} \mathrm{NaO}_{2}\left(\mathrm{M}^{+}+\mathrm{Na}\right)$ : 277.1199, Found: 277.1196.

(2) Methyl 2-(oct-1'-en-3'-yn-2'-yl)hex-2(E)-enoate (6bb) 


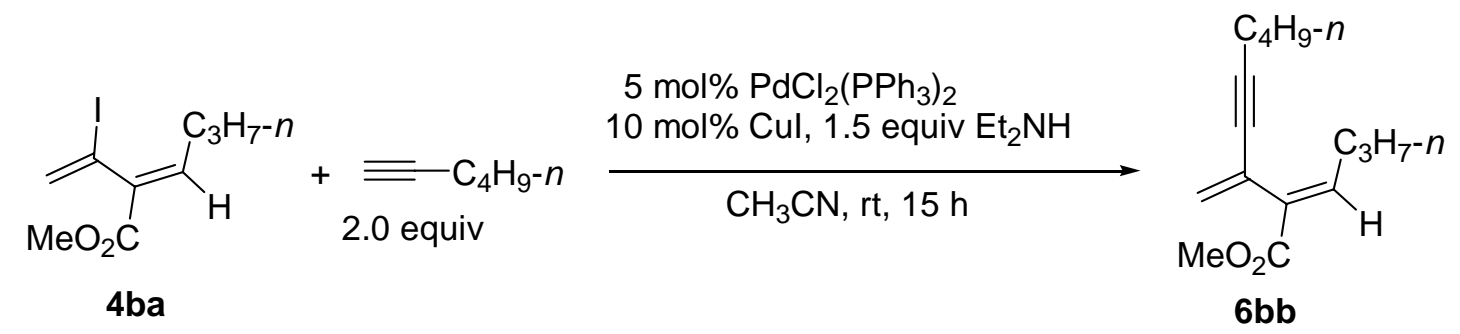

A mixture of $\mathrm{Pd}\left(\mathrm{PPh}_{3}\right)_{2} \mathrm{Cl}_{2}$ (7.1 mg, $\left.5 \mathrm{~mol} \%, 0.01 \mathrm{mmol}\right)$, CuI (3.9 mg, $10 \mathrm{~mol} \%$, $0.02 \mathrm{mmol}), \mathrm{Et}_{2} \mathrm{NH}$ (21.8 mg, $\left.0.30 \mathrm{mmol}\right), 1$-hexyne (33.0 mg, $\left.0.40 \mathrm{mmol}\right)$, and $4 \mathbf{b a}$ (57.3 mg, $0.20 \mathrm{mmol}$ ) in $\mathrm{CH}_{3} \mathrm{CN}\left(2 \mathrm{~mL}\right.$ ) afforded $\mathbf{6 b b}$ (30.6 mg, 64\%): Liquid; ${ }^{1} \mathrm{H}$ NMR (400 MHz, $\left.\mathrm{CDCl}_{3}\right) \delta 6.82(\mathrm{t}, J=7.8 \mathrm{~Hz}, 1 \mathrm{H}), 5.62$ (s, 1H), 5.19 (s, 1H), 3.73 (s, 3H), 2.31-2.18 (m, 4H), 1.51-1.41 (m, 4H), 1.40-1.33 (m, 2H), 0.96-0.84 (m, 6H); ${ }^{13} \mathrm{C}$ NMR (100 MHz, $\left.\mathrm{CDCl}_{3}\right): \delta 166.7,145.0,132.4,125.5,125.1,90.5,79.9,51.9,31.3$, 30.6, 21.9, 21.8, 19.0, 13.8, 13.5; MS (m/z): $234\left(\mathrm{M}^{+}, 100\right)$; IR (neat, $\left.\mathrm{cm}^{-1}\right)$ : 2959, 2933, 2222, 1723, 1640, 1598, 1461, 1434, 1249, 1058. HRMS calcd for $\mathrm{C}_{15} \mathrm{H}_{22} \mathrm{O}_{2}$ $\left(\mathrm{M}^{+}\right)$: 234.1614, Found: 234.1617.

(3) Methyl 2-(hex-5'-hydroxy-5'-methyl-1'-en-3'-yn-2'-yl)hex-2(E)-enoate (6bc)

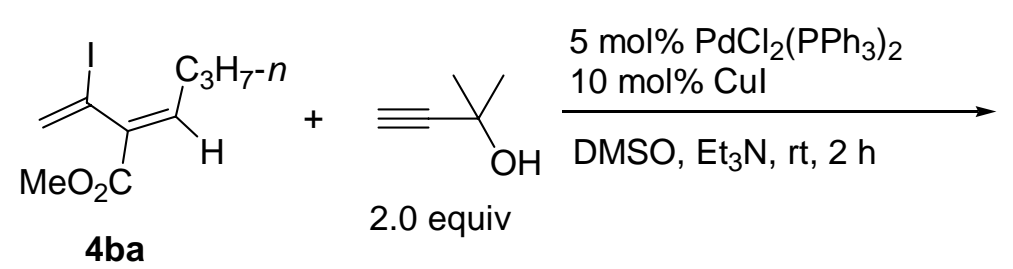

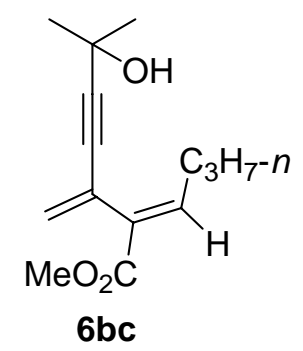

A mixture of $\mathrm{Pd}\left(\mathrm{PPh}_{3}\right)_{2} \mathrm{Cl}_{2}(7.1 \mathrm{mg}, 5 \mathrm{~mol} \%, 0.01 \mathrm{mmol})$, CuI (3.8 mg, $10 \mathrm{~mol} \%$, $0.02 \mathrm{mmol})$, 2-methyl-3-butyn-2-ol (33.5 mg, $0.40 \mathrm{mmol}), 4$ ba (55.8 mg, $0.20 \mathrm{mmol}$ ), and $\mathrm{Et}_{3} \mathrm{~N}(1 \mathrm{~mL})$ in DMSO $(1 \mathrm{~mL})$ was stirred at room temperature over a period of 2 h under nitrogen. After complete conversion of the starting materials as monitored by TLC, the reaction mixture was diluted with $10 \mathrm{~mL}$ of $\mathrm{Et}_{2} \mathrm{O}$ and quenched with $5 \mathrm{~mL}$ 
of water. The organic layer was separated and the aqueous layer was extracted with diethyl ether ( 3 x $20 \mathrm{~mL}$ ). The combined organic layer was washed with brine and dried over $\mathrm{Na}_{2} \mathrm{SO}_{4}$. Evaporation and column chromatography on silica gel (petroleum ether/ethyl acetate $=8: 1$, then 4:1) afforded $\mathbf{6 b c}(32.5 \mathrm{mg}, 69 \%)$ : Liquid; ${ }^{1} \mathrm{H}$ NMR $\left(400 \mathrm{MHz}, \mathrm{CDCl}_{3}\right) \delta 6.82(\mathrm{t}, J=7.6 \mathrm{~Hz}, 1 \mathrm{H}), 5.67$ (d, $\left.J=1.6 \mathrm{~Hz}, 1 \mathrm{H}\right), 5.25$ (d, $J=$ $1.6 \mathrm{~Hz}, 1 \mathrm{H}), 3.71$ (s, 3H), 2.78-2.69 (bs, 1H), 2.20 (q, $J=7.6 \mathrm{~Hz}, 2 \mathrm{H}), 1.51-1.37$ (m, 2H), 1.46 (s, 6H), 0.89 (t, $J=7.6 \mathrm{~Hz}, 3 \mathrm{H}) ;{ }^{13} \mathrm{C}$ NMR (100 MHz, $\left.\mathrm{CDCl}_{3}\right): \delta$ 166.5, 145.6, 131.7, 126.7, 124.6, 93.6, 81.3, 65.3, 51.9, 31.4, 31.2, 21.9, 13.8; MS (m/z): $236\left(\mathrm{M}^{+}, 77.92\right), 189$ (100); IR (neat, $\left.\mathrm{cm}^{-1}\right)$ : 3460, 2961, 1722, 1640, 1597, 1436, 1366, 1253, 1059. HRMS calcd for $\mathrm{C}_{14} \mathrm{H}_{20} \mathrm{O}_{3}\left(\mathrm{M}^{+}\right)$: 236.1415, Found: 236.1415.

(4) Methyl 2-(1'-phenylvinyl)hex-2(E)-enoate (6bd)

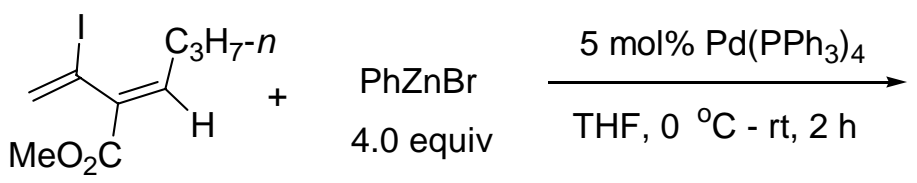

4ba<smiles>C=C(C(C)=O)C(=CCCCCC)c1ccccc1</smiles>

6bd

To a solution of $\mathrm{ZnBr}_{2}(270.5 \mathrm{mg}, 1.20 \mathrm{mmol})$ in $2 \mathrm{~mL}$ of THF was added $1.2 \mathrm{~mL}$ of phenylmagnesium bromide (1M, $1.2 \mathrm{mmol}$ ) prepared from magnesium (384.7 mg, $16 \mathrm{mmol}$ ) and bromobenzene (2.5120 g, $16 \mathrm{mmol})$ in $16 \mathrm{~mL}$ of THF by a syringe at 0 ${ }^{\circ} \mathrm{C}$ under nitrogen. After the addition, the reaction was stirred and warmed up to room temperature naturally. To a solution of $\mathrm{Pd}\left(\mathrm{PPh}_{3}\right)_{4}(17.8 \mathrm{mg}, 0.015 \mathrm{mmol})$ and $4 \mathbf{b a}$ (86.1 mg, 0.31mmol) in $2 \mathrm{~mL}$ of THF was added the above prepared solution of phenylzinc bromide (cooled to $0{ }^{\circ} \mathrm{C}$ and then transferred quickly via a syringe) at $0{ }^{\circ} \mathrm{C}$ 
under nitrogen. The reaction mixture was warmed up to room temperature naturally. After $2 \mathrm{~h}$, the reaction was complete as monitored by TLC, quenched with $5 \mathrm{~mL}$ of water, extracted with diethyl ether (3 x $25 \mathrm{~mL}$ ). The combined organic layer was washed with brine and dried over anhydrous $\mathrm{Na}_{2} \mathrm{SO}_{4}$. Evaporation and column chromatography on silica gel (petroleum ether/ diethyl ether $=30: 1$ ) afforded 6 bd (66.3 mg, 94\%): Liquid; ${ }^{1} \mathrm{H}$ NMR (400 MHz, $\mathrm{CDCl}_{3}$ ) $\delta$ 7.41-7.37 (m, 2H), 7.36-7.25 (m, 3H), 7.13 (t, $J=7.6 \mathrm{~Hz}, 1 \mathrm{H}), 5.81$ (s, 1H), 5.14 (s, 1H), 3.65 (s, 3H), 2.22 (q, $J=$ $7.5 \mathrm{~Hz}, 2 \mathrm{H}), 1.54-1.43$ (m, 2H), 0.94 (t, $J=7.4 \mathrm{~Hz}, 3 \mathrm{H}) ;{ }^{13} \mathrm{C}$ NMR (100 MHz, $\left.\mathrm{CDCl}_{3}\right)$ : $\delta 167.4,146.1,142.5,139.2,133.4,128.3,127.7,125.7,116.2,51.8,31.5,22.1,13.9$; MS (m/z): 230 ( $\left.\mathrm{M}^{+}, 85.47\right), 171$ (100); IR (neat, $\mathrm{cm}^{-1}$ ): 2959, 2931, 1720, 1638, 1614, 1247. HRMS calcd for $\mathrm{C}_{15} \mathrm{H}_{18} \mathrm{NaO}_{2}\left(\mathrm{M}^{+}+\mathrm{Na}\right)$ : 253.1199, Found: 253.1192.

(5) Methyl 2-(1',3'(E)-octadien-2'-yl)hex-2(E)-enoate (6be)

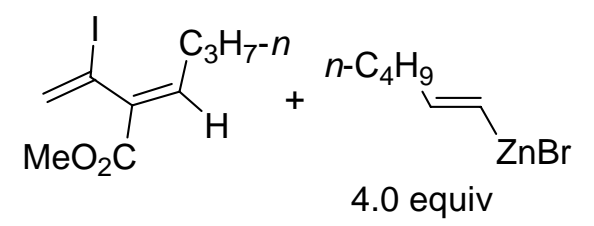

4ba

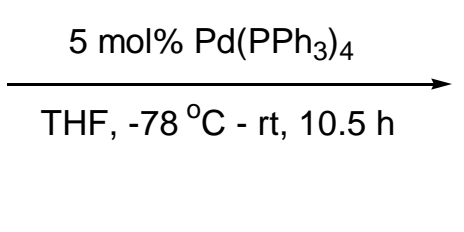

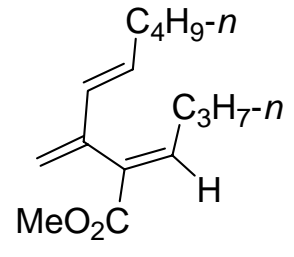

6be

To a solution of $1(E)$-hexenyl iodide (255.7 mg, $1.22 \mathrm{mmol})$ in THF (2 mL) was added a solution of $n$-BuLi ( $0.48 \mathrm{~mL}, 2.5 \mathrm{M}$ in hexanes, $1.20 \mathrm{mmol})$ at $-78{ }^{\circ} \mathrm{C}$. After 1 h at $-40{ }^{\circ} \mathrm{C}$, a solution of $\mathrm{ZnBr}_{2}$ (338 $\mathrm{mg}, 1.5 \mathrm{mmol}$ ) in $2.5 \mathrm{~mL}$ of THF was added by a syringe. After the addition, the reaction mixture was stirred for another $10 \mathrm{~min}$ at $-40{ }^{\circ} \mathrm{C}$, warmed up to room temperature naturally and kept at rt for $30 \mathrm{~min}$. Then $\mathrm{Pd}\left(\mathrm{PPh}_{3}\right)_{4}(18.1 \mathrm{mg}, 0.015 \mathrm{mmol}), 4$ ba $(84.6 \mathrm{mg}, 0.30 \mathrm{mmol})$, and $2 \mathrm{~mL}$ of $\mathrm{THF}$ 
were added subsequently under nitrogen. After $10.5 \mathrm{~h}$, the reaction was complete as monitored by TLC, quenched with $5 \mathrm{~mL}$ of dilute aqueous $\mathrm{HCl}$ (5\%), extracted with diethyl ether (3 x $25 \mathrm{~mL}$ ), and dried over $\mathrm{Na}_{2} \mathrm{SO}_{4}$. Evaporation and column chromatography on silica gel (petroleum ether/diethyl ether $=50: 1$ ) afforded 6be (55.5 mg, 78\%): Liquid; ${ }^{1} \mathrm{H}$ NMR (400 MHz, $\mathrm{CDCl}_{3}$ ) $\delta 6.95$ (t, $\left.J=7.4 \mathrm{~Hz}, 1 \mathrm{H}\right), 6.14$ (d, $J=16.0 \mathrm{~Hz}, 1 \mathrm{H}$ ), 5.42 (dt, $J_{1}=16.0 \mathrm{~Hz}, J_{2}=6.8 \mathrm{~Hz}, 1 \mathrm{H}$ ), 5.24-5.20 (bs, $1 \mathrm{H}$ ), 4.83 (d, $J=1.2 \mathrm{~Hz}, 1 \mathrm{H}), 3.69$ (s, 3H), 2.11-2.03 (m, 4H), 1.45-1.37 (m, 2H), 1.37-1.20 (m, 4H), 0.92-0.81 (m, 6H); ${ }^{13} \mathrm{C}$ NMR (100 MHz, $\left.\mathrm{CDCl}_{3}\right): \delta 167.4,145.5,141.9,133.3$, 132.0, 130.8, 116.6, 51.8, 32.1, 31.3, 31.2, 22.1, 22.0, 13.8; MS (m/z): $236\left(\mathrm{M}^{+}\right.$, 75.20), 107 (100); IR (neat, $\mathrm{cm}^{-1}$ ): 2958, 2930, 1721, 1641, 1597, 1247. HRMS calcd for $\mathrm{C}_{15} \mathrm{H}_{25} \mathrm{O}_{2}\left(\mathrm{M}^{+}+\mathrm{H}\right)$ : 237.1849, Found: 237.1848.

(6) Methyl 2-(1'-hexen-2'-yl)hex-2(E)-enoate (6bf)

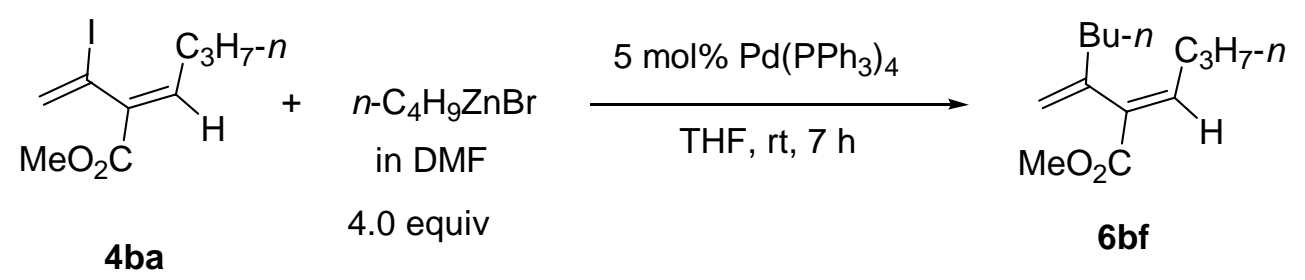

To a solution of $n$-BuLi $(0.48 \mathrm{~mL}, 2.5 \mathrm{M}$ in hexanes, $1.20 \mathrm{mmol})$ at $-78{ }^{\circ} \mathrm{C}$ was added a solution of $\mathrm{ZnBr}_{2}$ (338 $\mathrm{mg}, 1.5 \mathrm{mmol}$ ) in $2 \mathrm{~mL}$ of THF by a syringe. After the addition, the reaction mixture was stirred and warmed up to room temperature slowly. After the addition of anhydrous DMF (1 mL), the reaction mixture was stirred and transferred quickly into a solution of $\mathrm{Pd}\left(\mathrm{PPh}_{3}\right)_{4}(17.8 \mathrm{mg}, 0.015 \mathrm{mmol})$ and $\mathbf{4 b a}(84.2$ $\mathrm{mg}, 0.30 \mathrm{mmol}$ ) in $2 \mathrm{~mL}$ of THF under nitrogen. After $7 \mathrm{~h}$, the reaction was complete as monitored by TLC. Then it was quenched with $5 \mathrm{~mL}$ of $\mathrm{H}_{2} \mathrm{O}$ and $5 \mathrm{~mL}$ of saturated 
aqueous $\mathrm{NH}_{4} \mathrm{Cl}$, extracted with diethyl ether (3 x $25 \mathrm{~mL}$ ), and dried over $\mathrm{Na}_{2} \mathrm{SO}_{4}$. Evaporation and column chromatography on silica gel (petroleum ether/diethyl ether = 20:1) afforded $6 \mathbf{b f}$ (32.0 mg, 51\%): Liquid; ${ }^{1} \mathrm{H}$ NMR (400 MHz, $\left.\mathrm{CDCl}_{3}\right) \delta 6.83$ (t, $J$ $=7.4 \mathrm{~Hz}, 1 \mathrm{H}), 5.17-5.11(\mathrm{~m}, 1 \mathrm{H}), 4.80-4.76(\mathrm{~m}, 1 \mathrm{H}), 3.73(\mathrm{~s}, 3 \mathrm{H}), 2.23-2.12(\mathrm{~m}, 4 \mathrm{H})$, 1.49-1.41 (m, 2H), 1.38-1.25 (m, 4H), 0.97-0.84 (m, 6H); ${ }^{13} \mathrm{C}$ NMR (100 MHz, $\left.\mathrm{CDCl}_{3}\right): \delta 167.5,144.5,144.3,134.7,115.1,51.7,36.0,31.5,29.8,22.5,22.3,13.9$; MS (m/z): 210 ( $\left.\mathrm{M}^{+}, 26.34\right), 181$ (100); IR (neat, $\mathrm{cm}^{-1}$ ): 2958, 2930, 1720, 1628, 1461, 1434, 1245. HRMS calcd for $\mathrm{C}_{13} \mathrm{H}_{23} \mathrm{O}_{2}\left(\mathrm{M}^{+}+\mathrm{H}\right)$ : 211.1693, Found: 211.1687.

\section{References:}

1. Winkler, J. D.; Quinn, K. J.; Mackinnon, C. H.; Hiscock, S. D.; Mclaughlin, E. C. Org. Lett. 2003, 5, 1805.

2. (a) Larock, R. C.; Liu, C. J. Org. Chem. 1983, 48, 2151; (b) Maclnnes, I.; Walton, J. C. J. Chem. Soc., Perkin Trans. II, 1987, 1077.

3. Mukaiyama, T.; Harada, T. Chem. Lett. 1981, 621. 


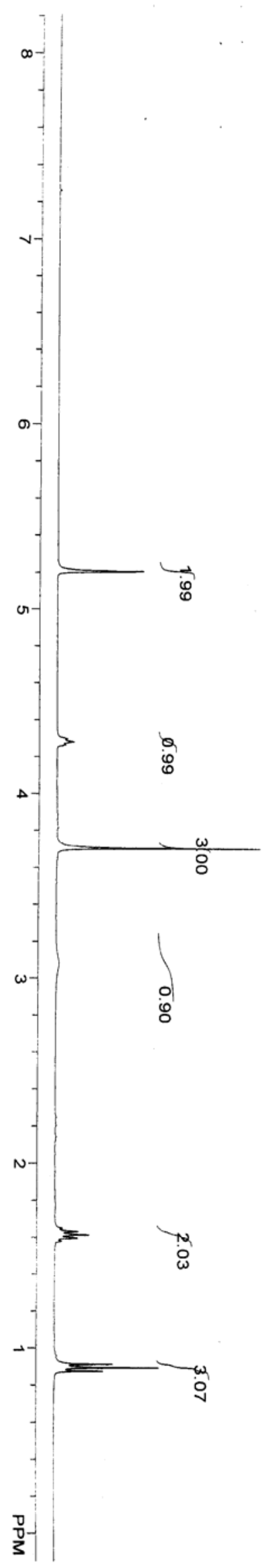

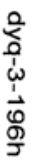

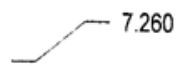

$\overbrace{\substack{0 \\ 0}}^{2}$
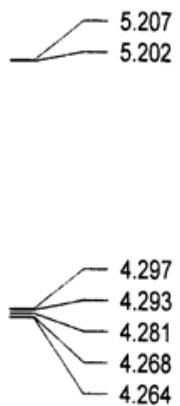

3.703

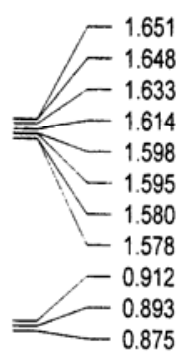



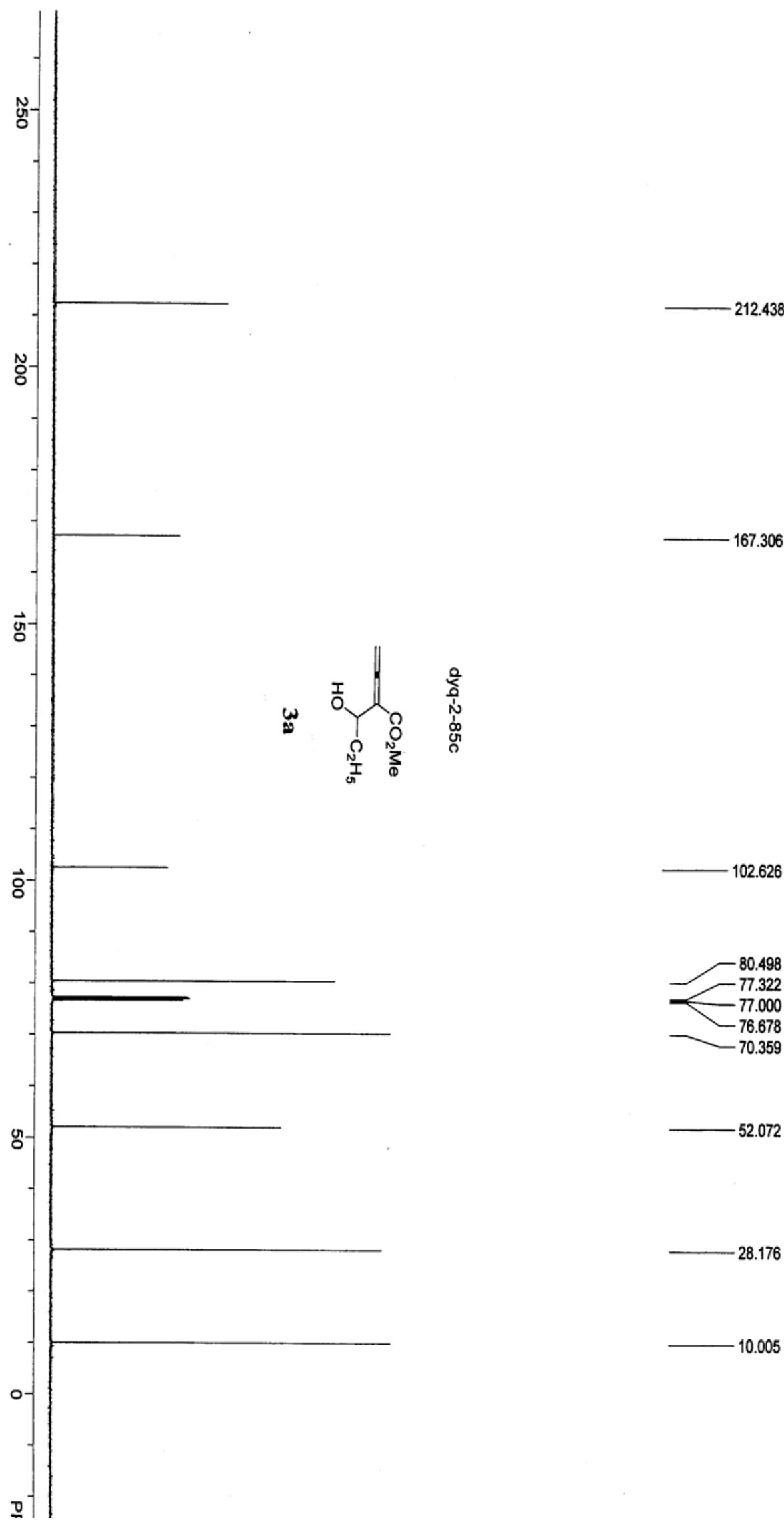

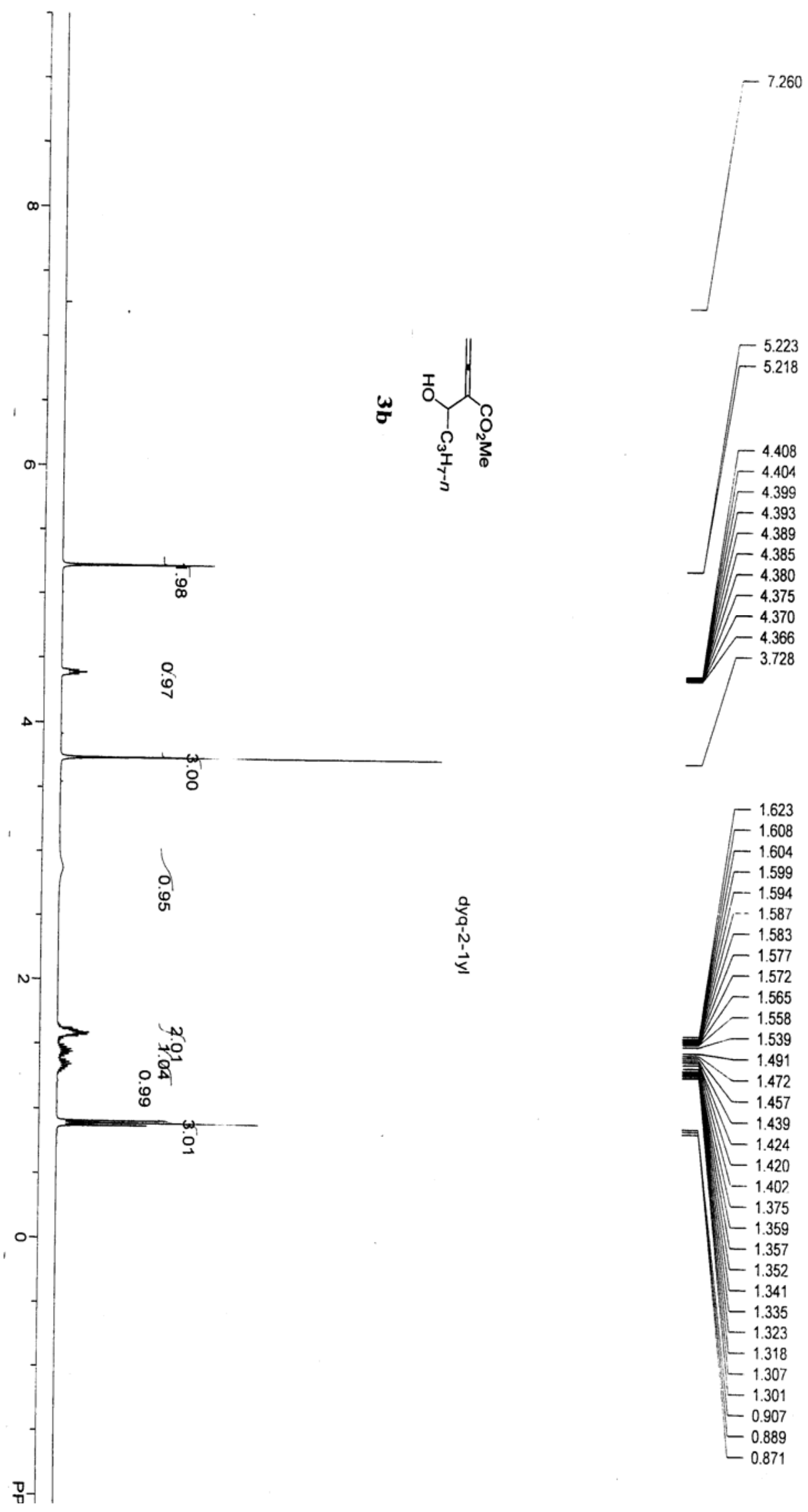

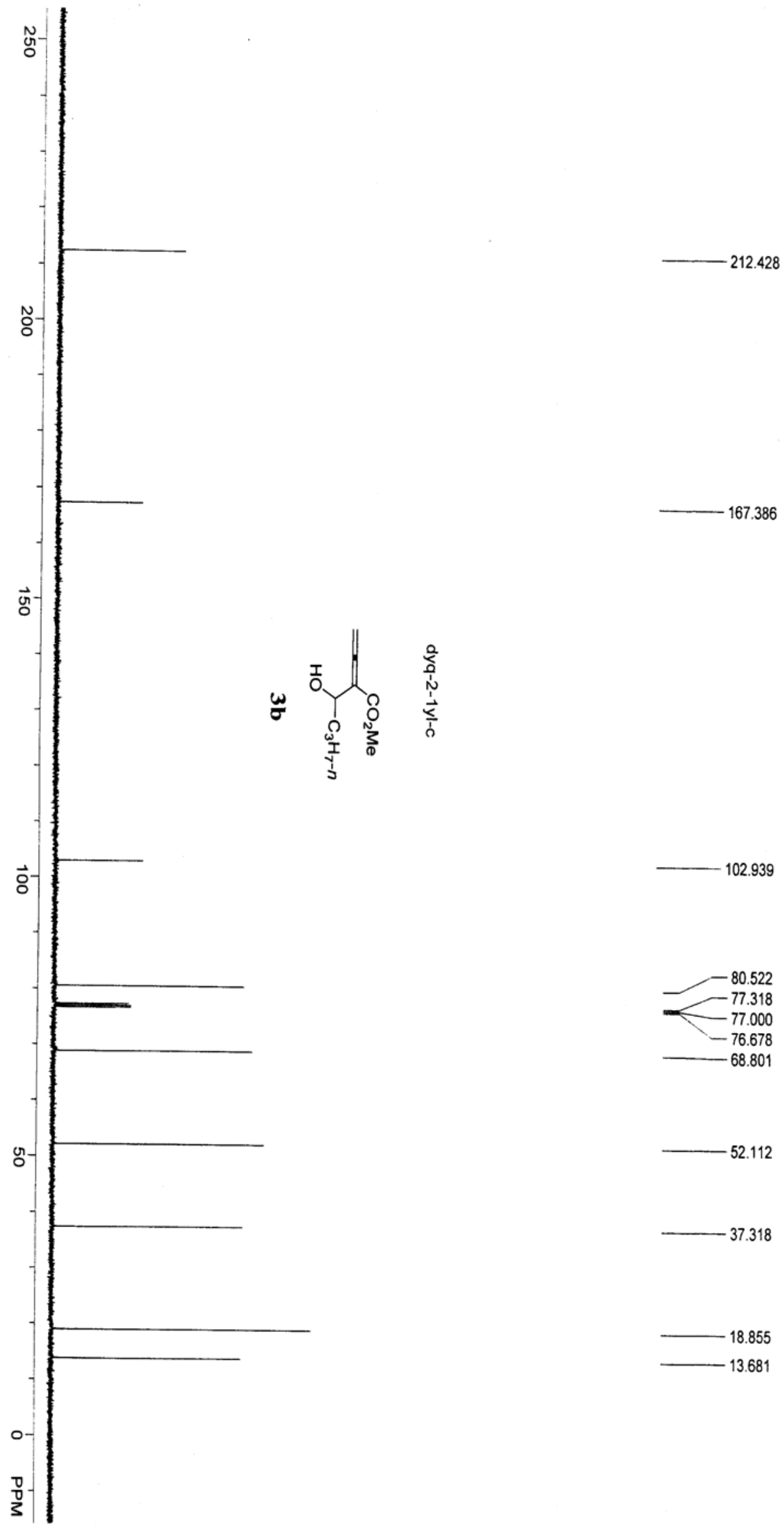

167.386
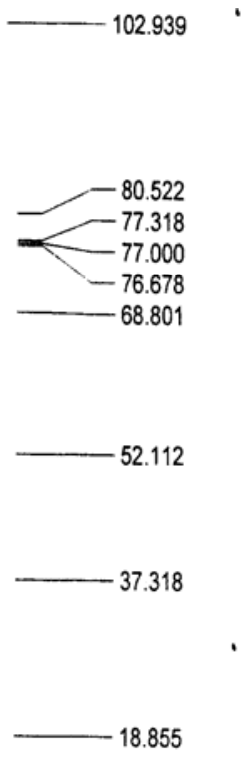

13.681 


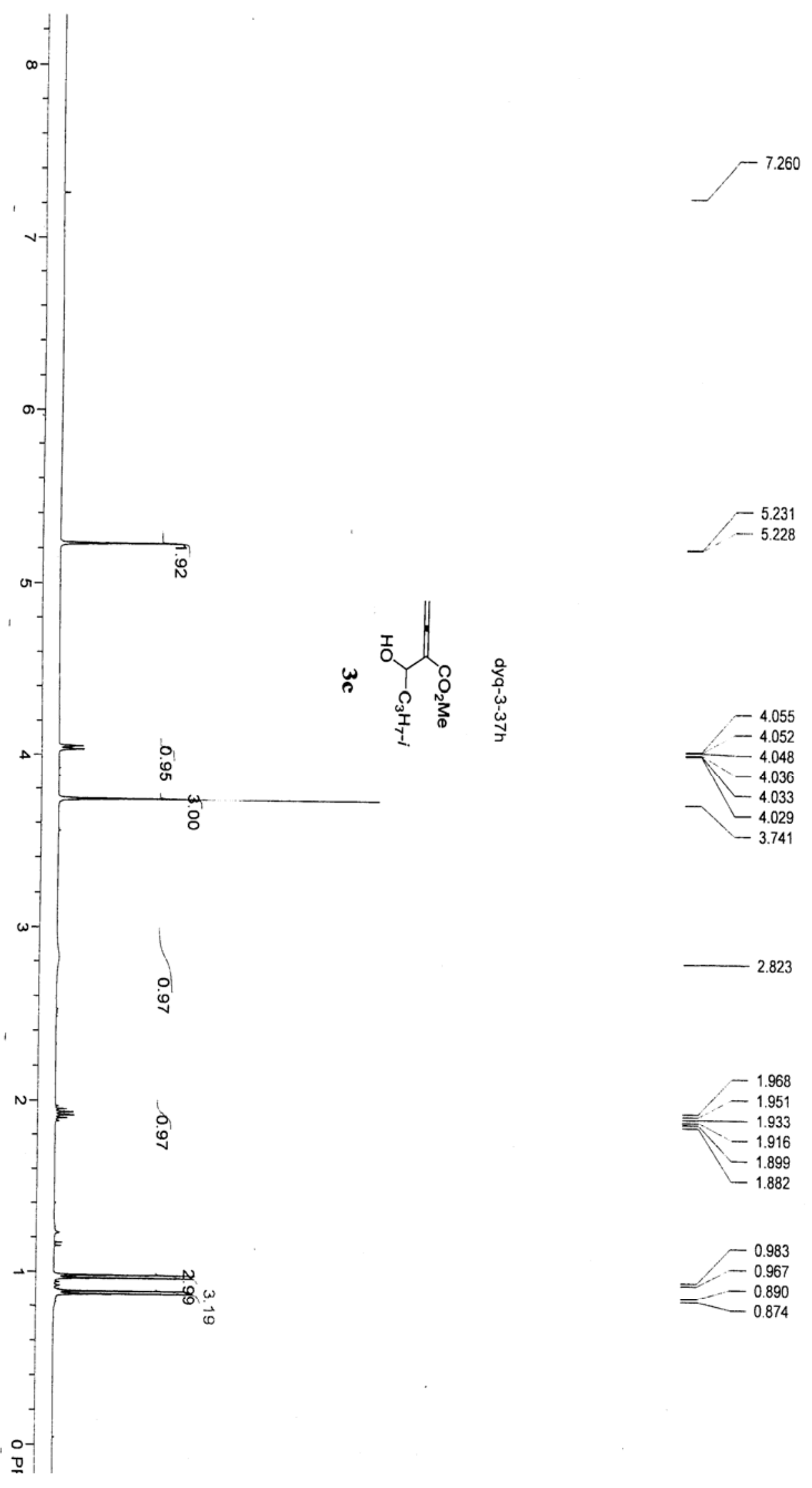



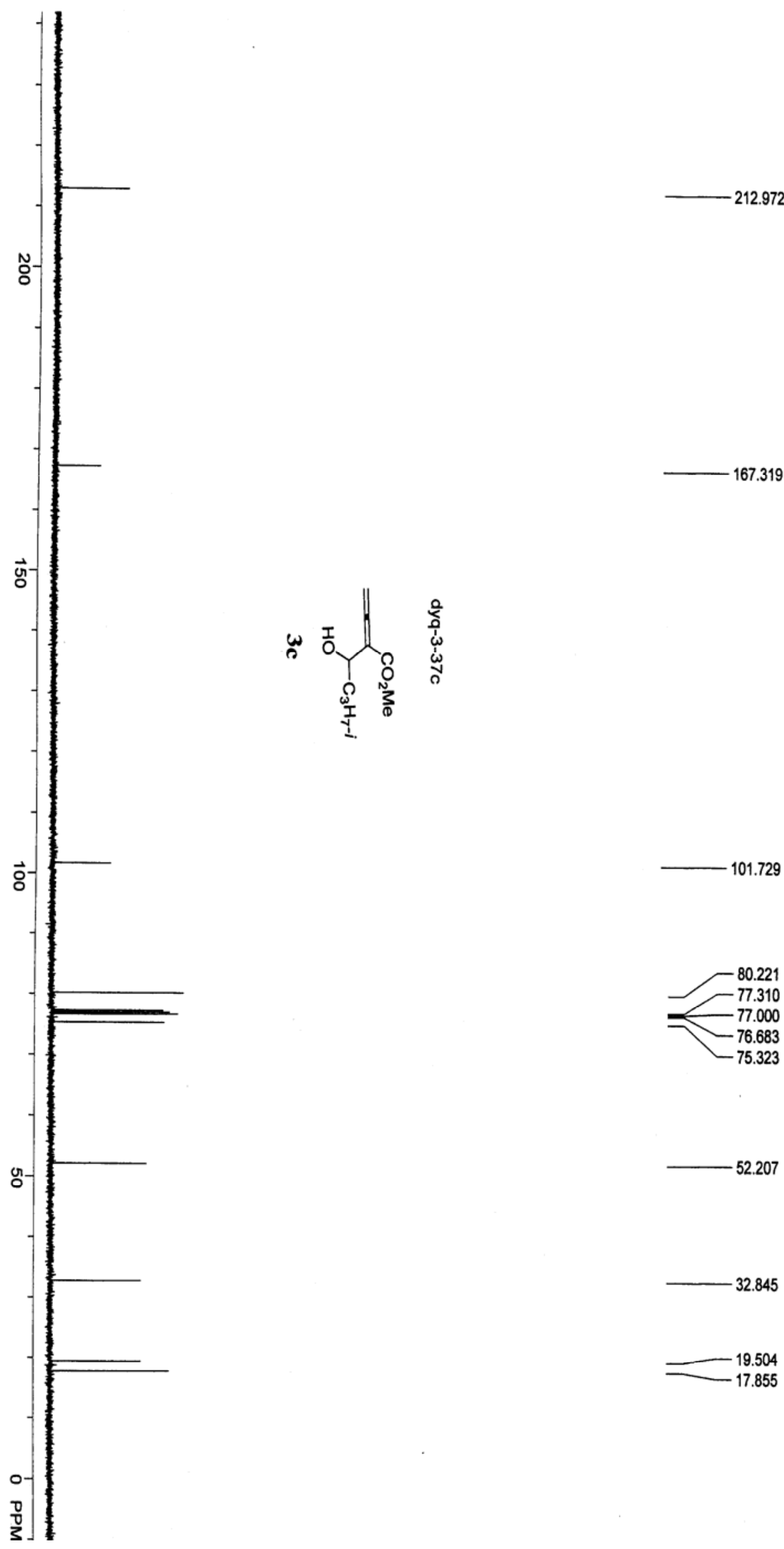

م.
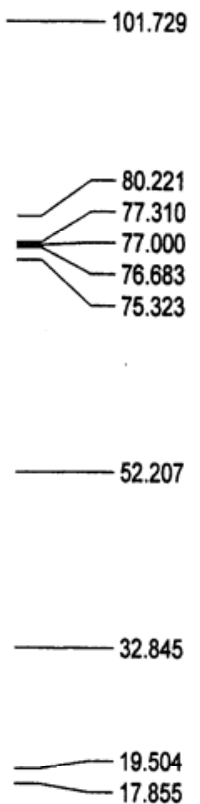

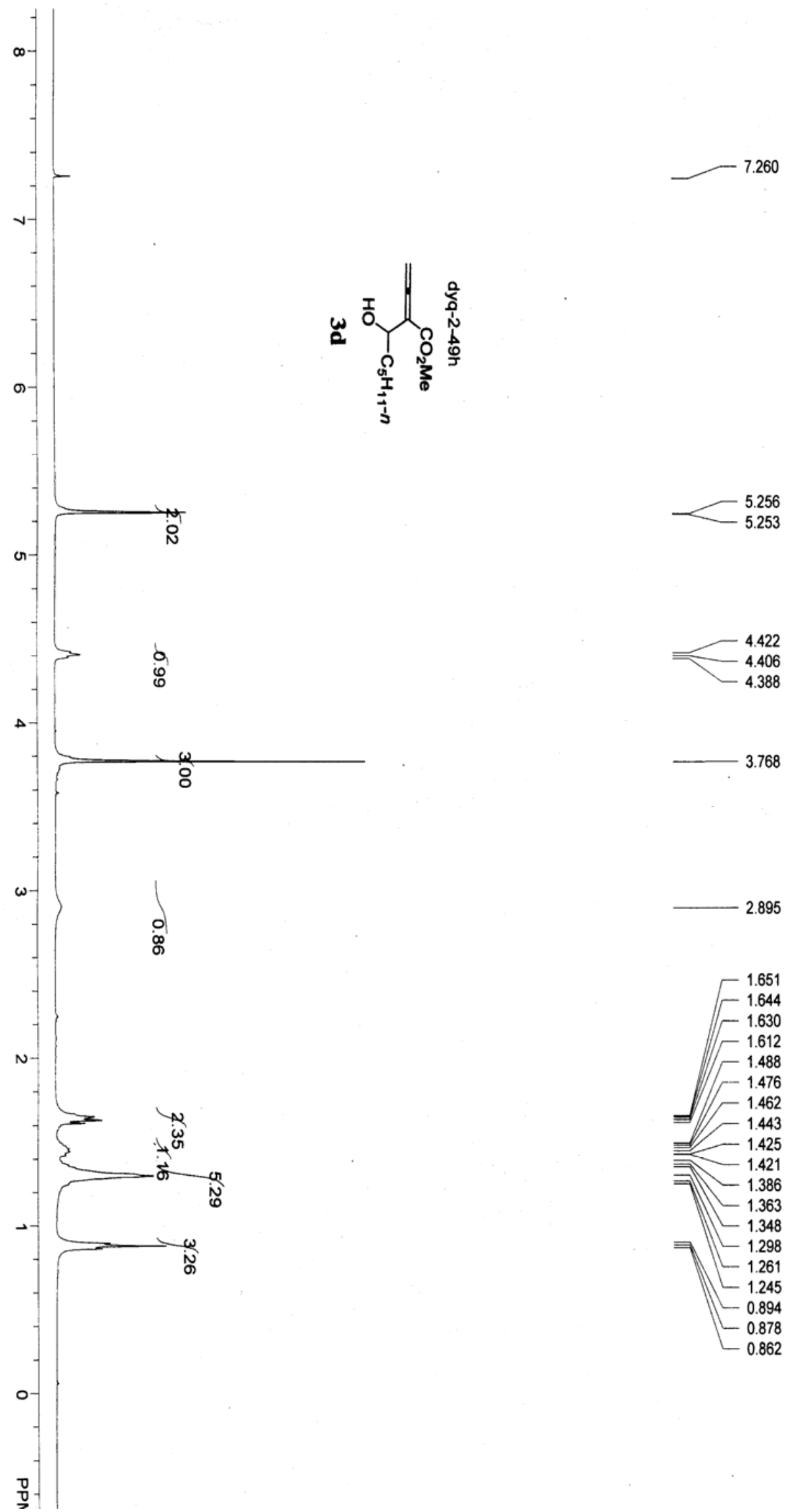

2.895

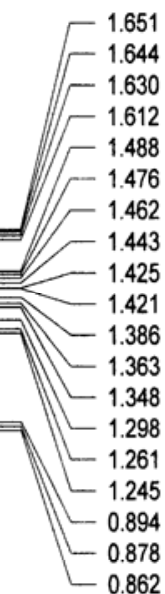



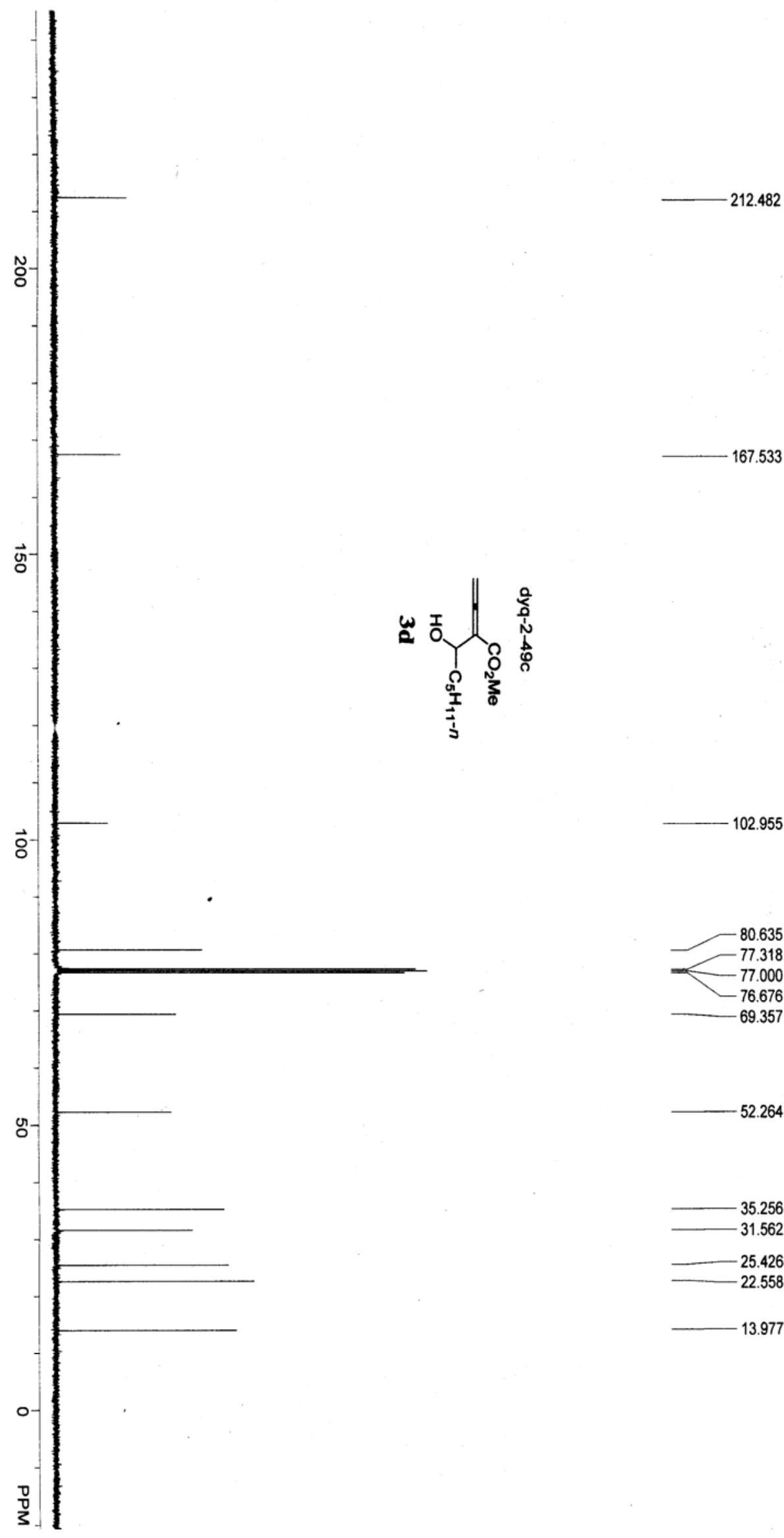

102.955

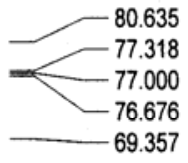

52.264

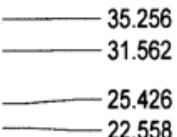

$-13.977$ 

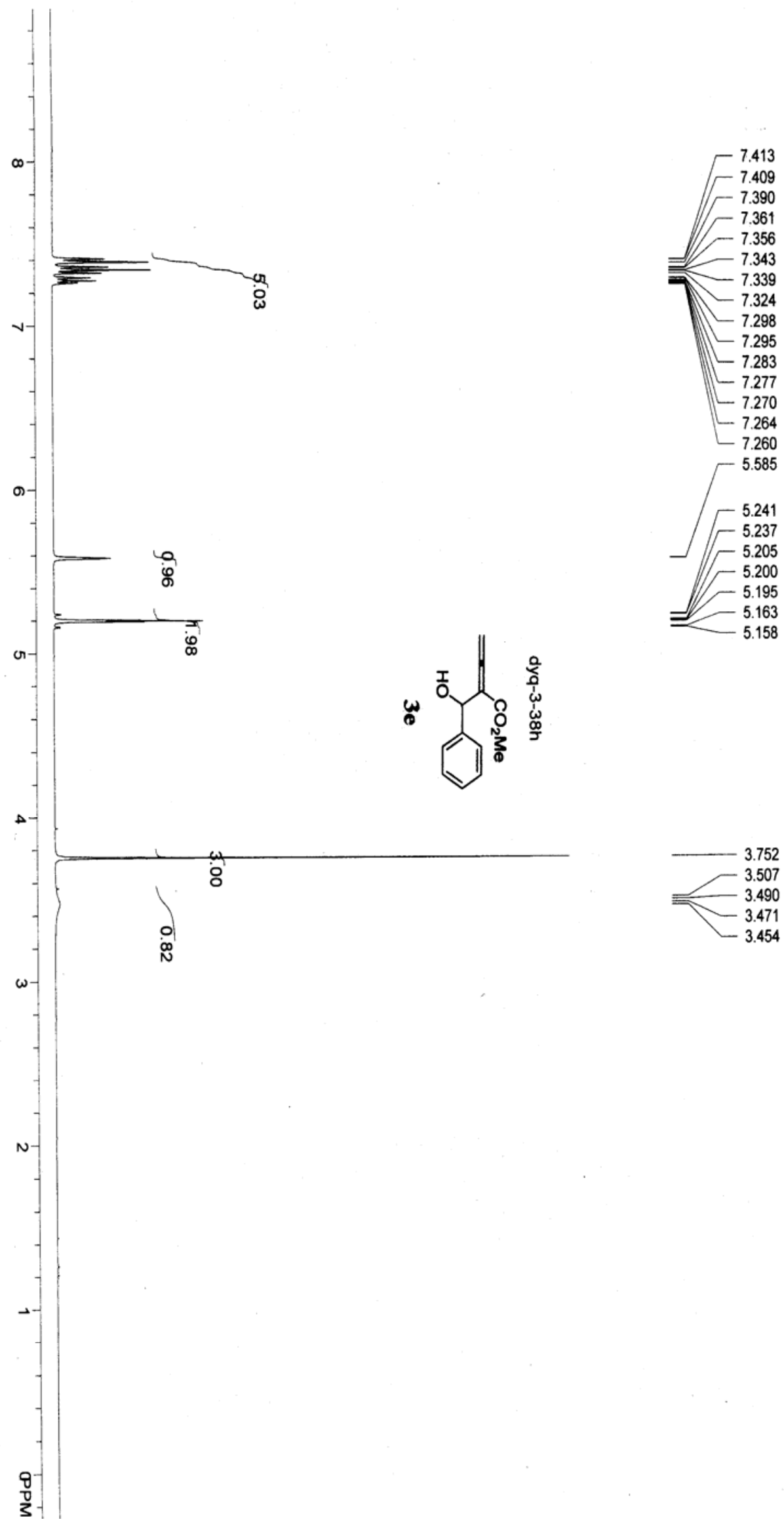


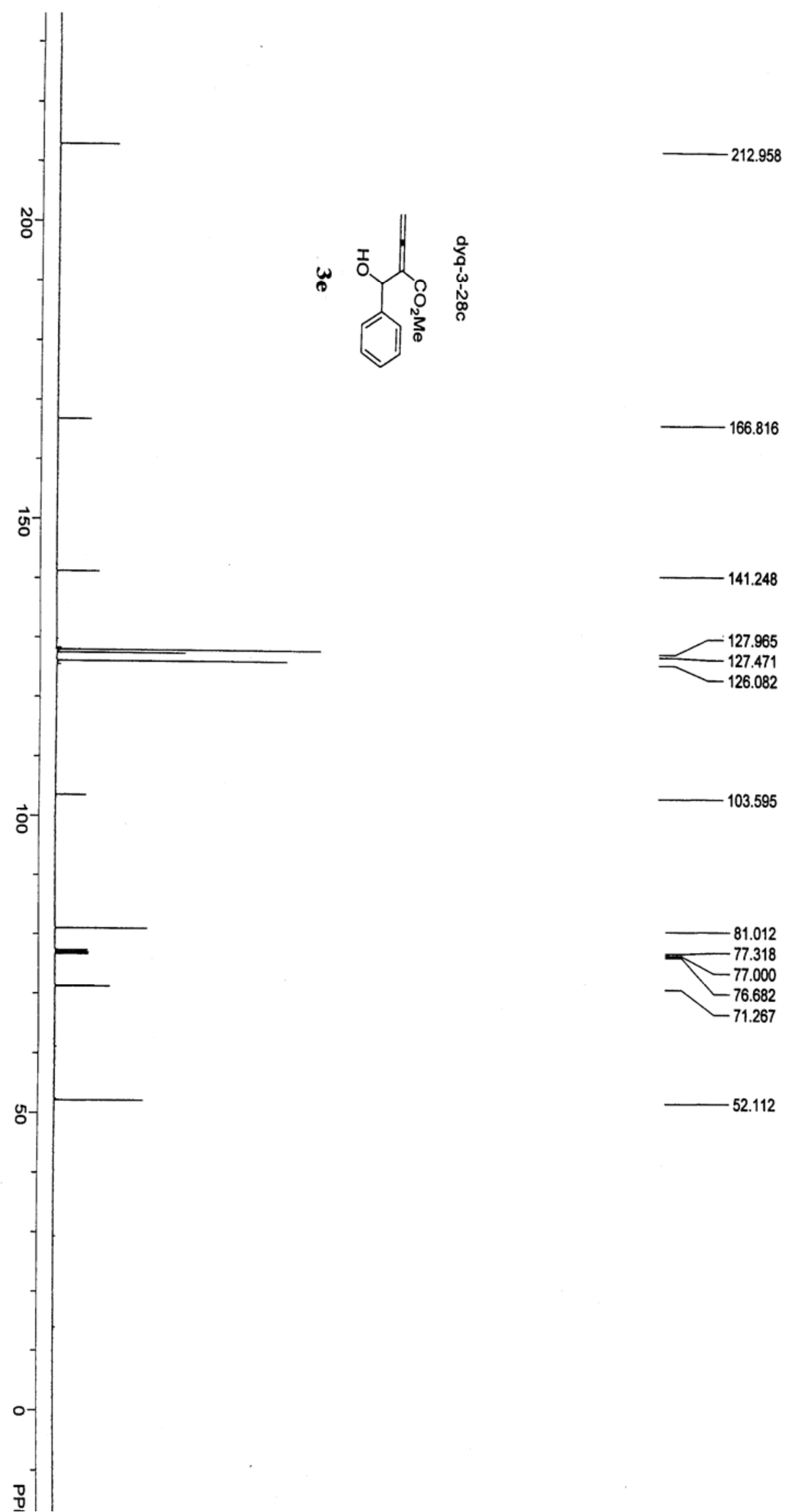



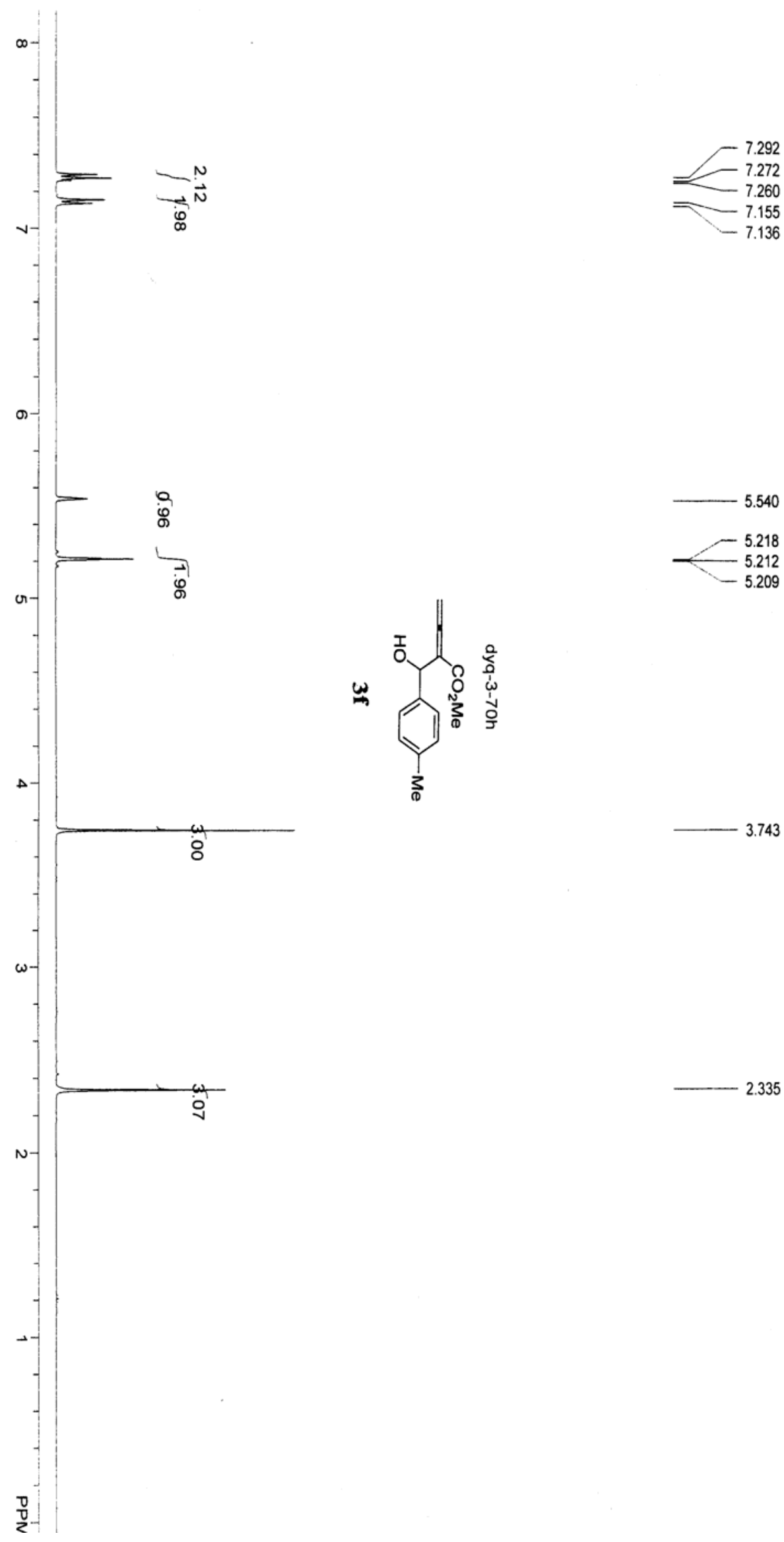


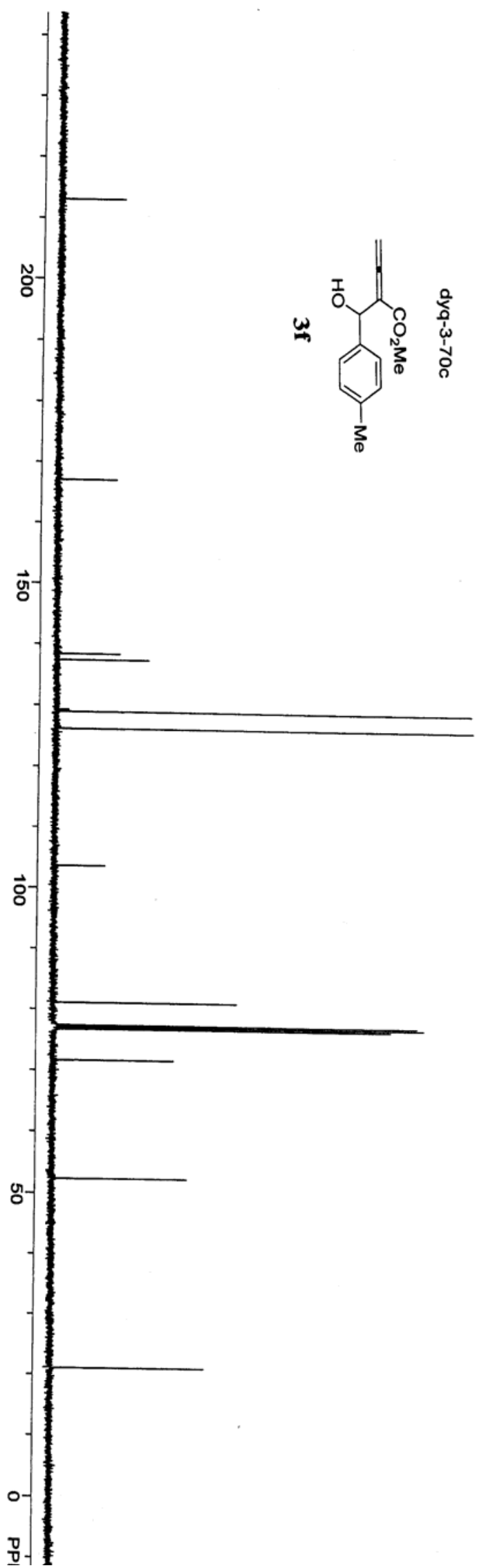

213.018
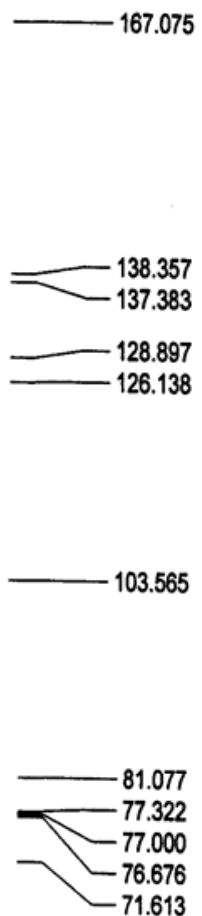

52.323

21.106 

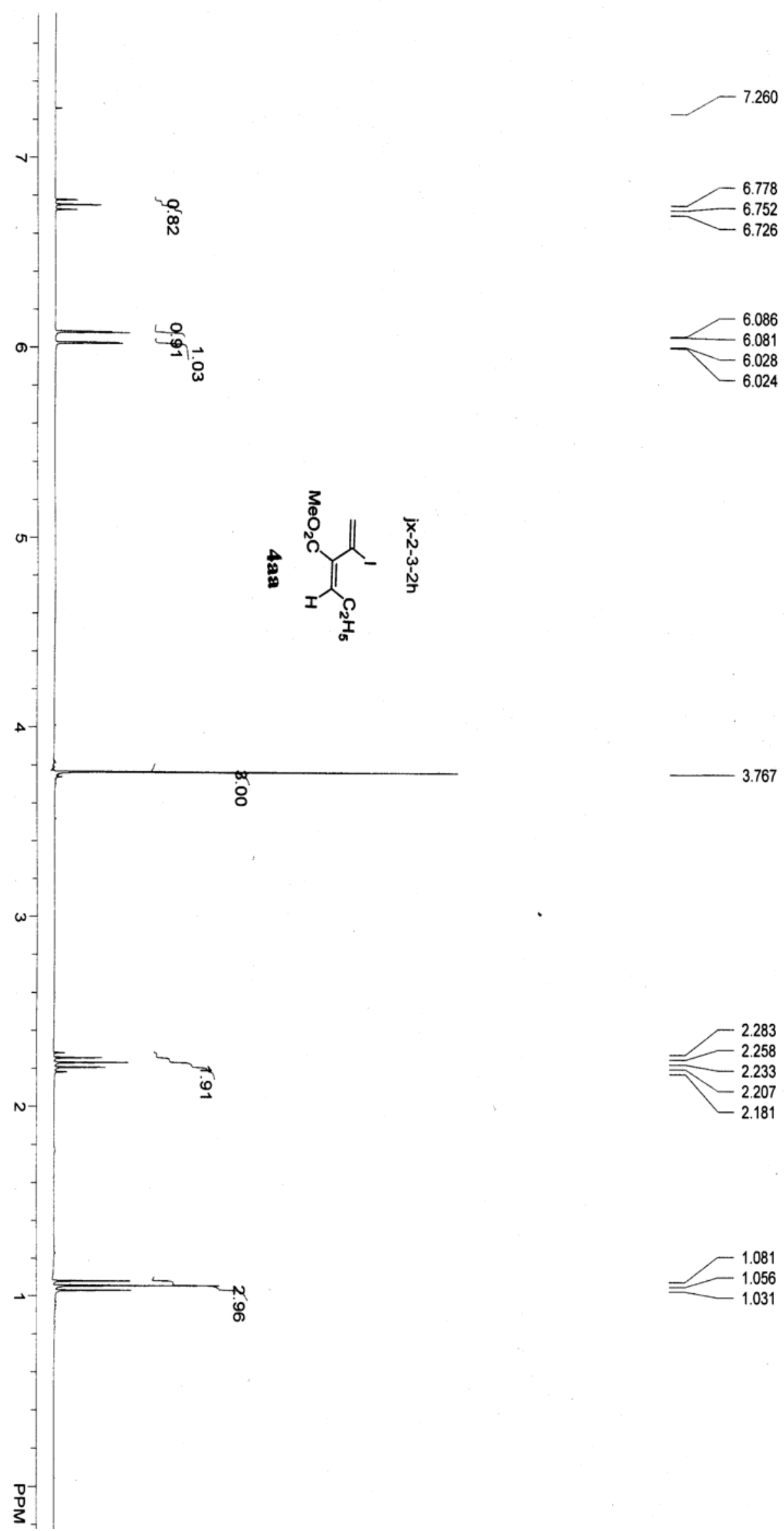

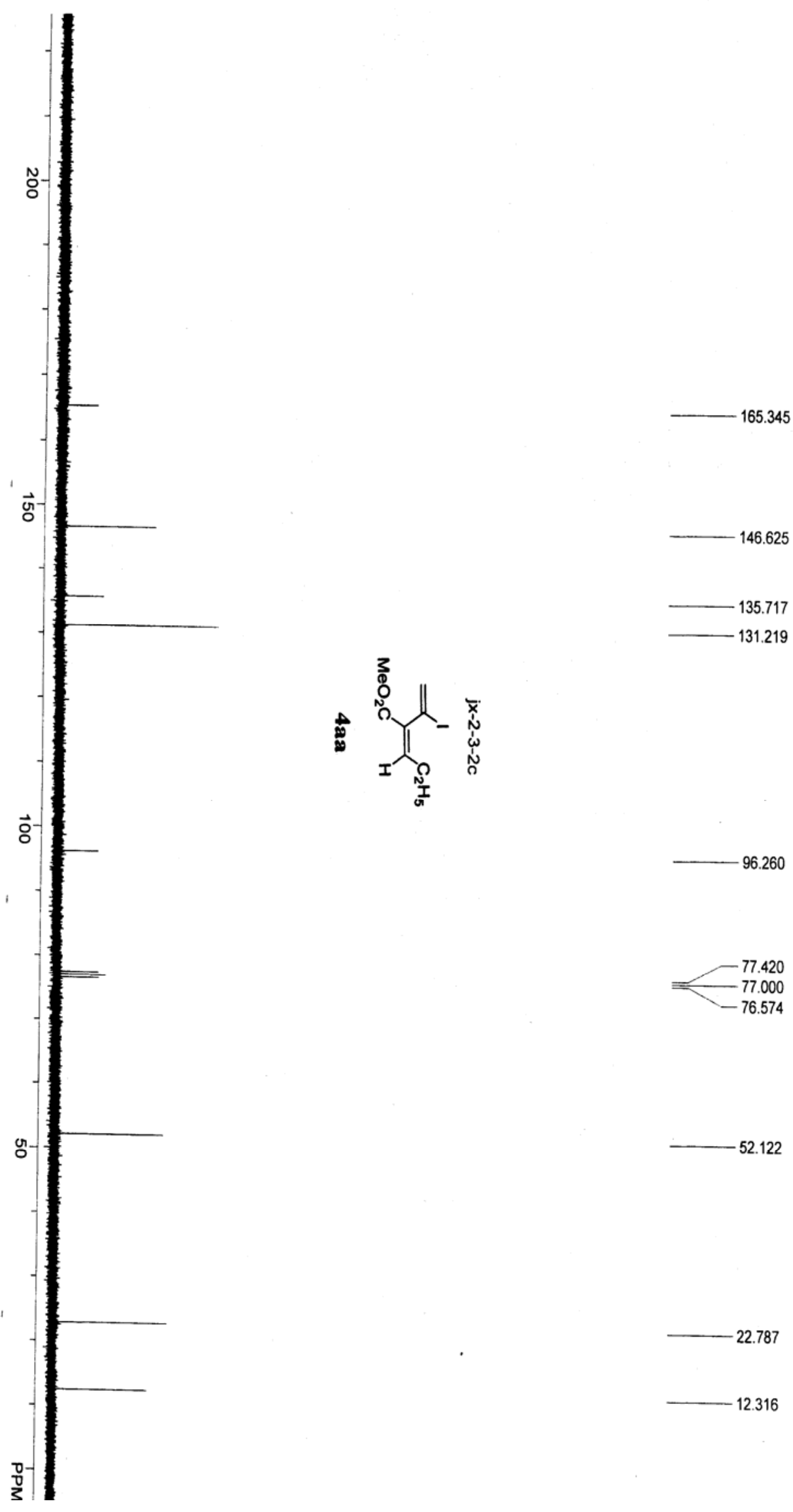

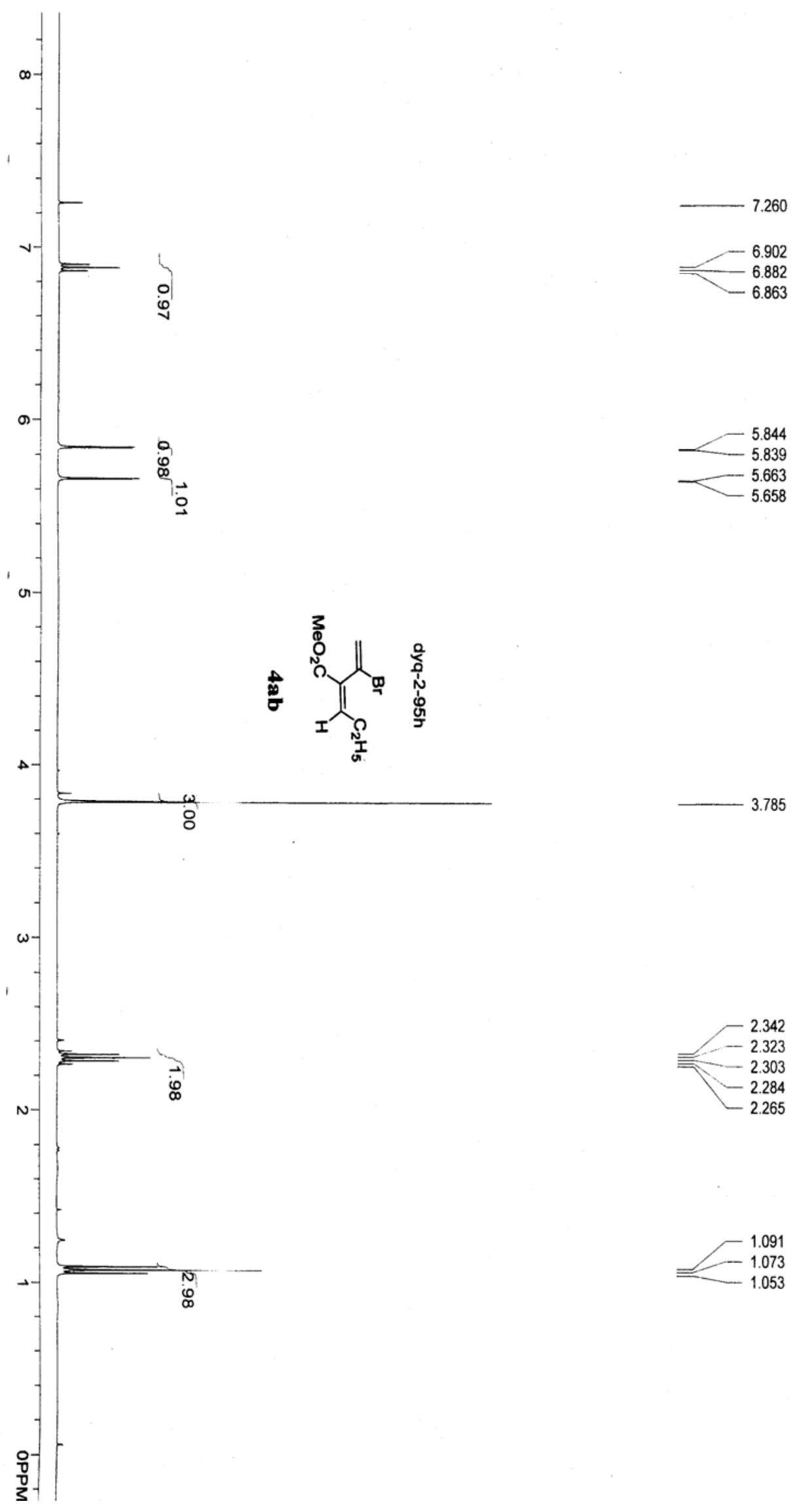

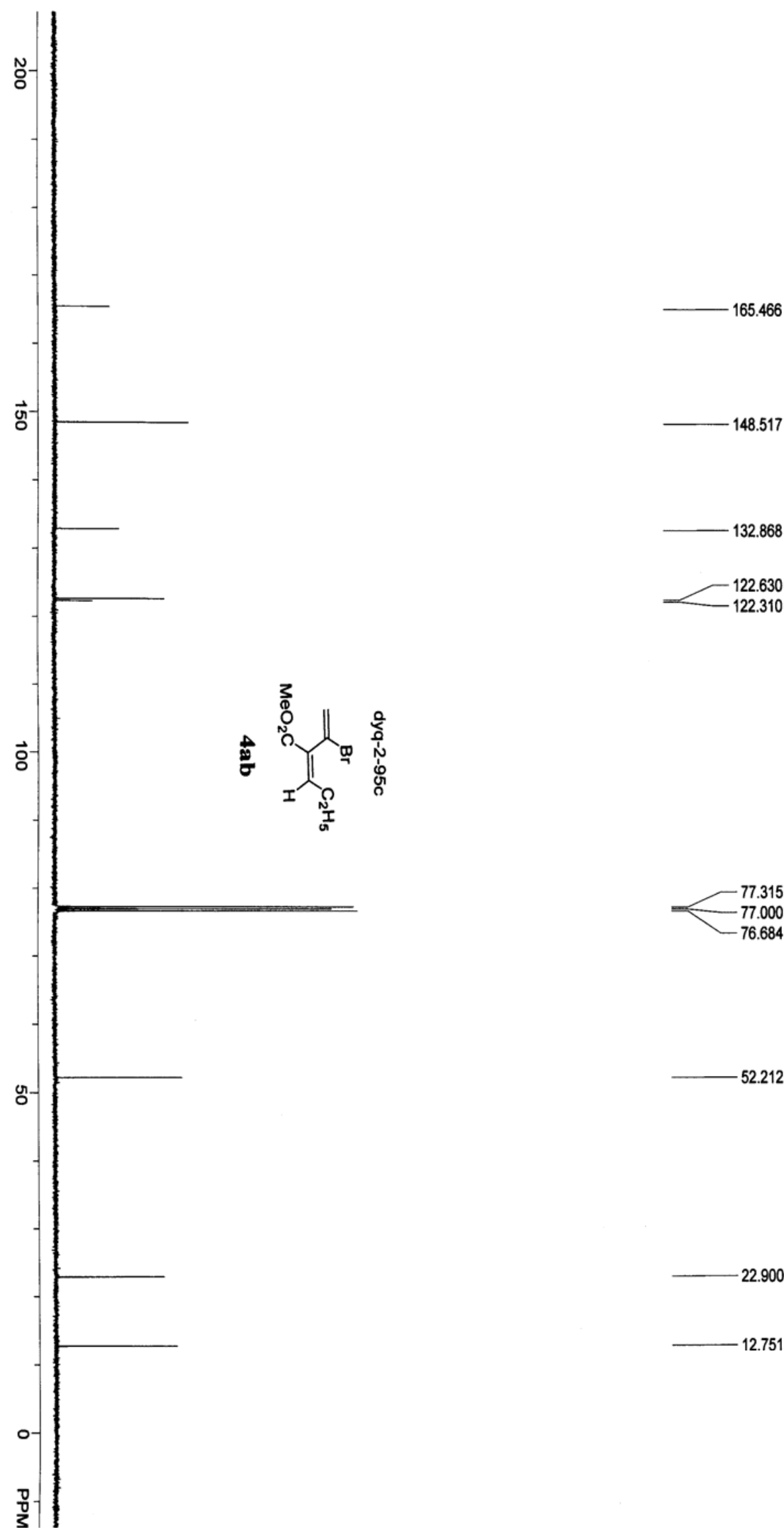

132.868
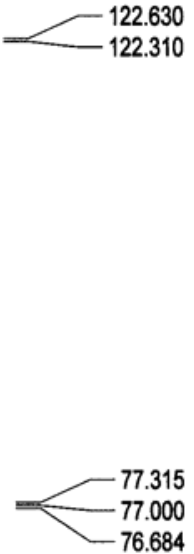

52.212

22.900

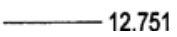



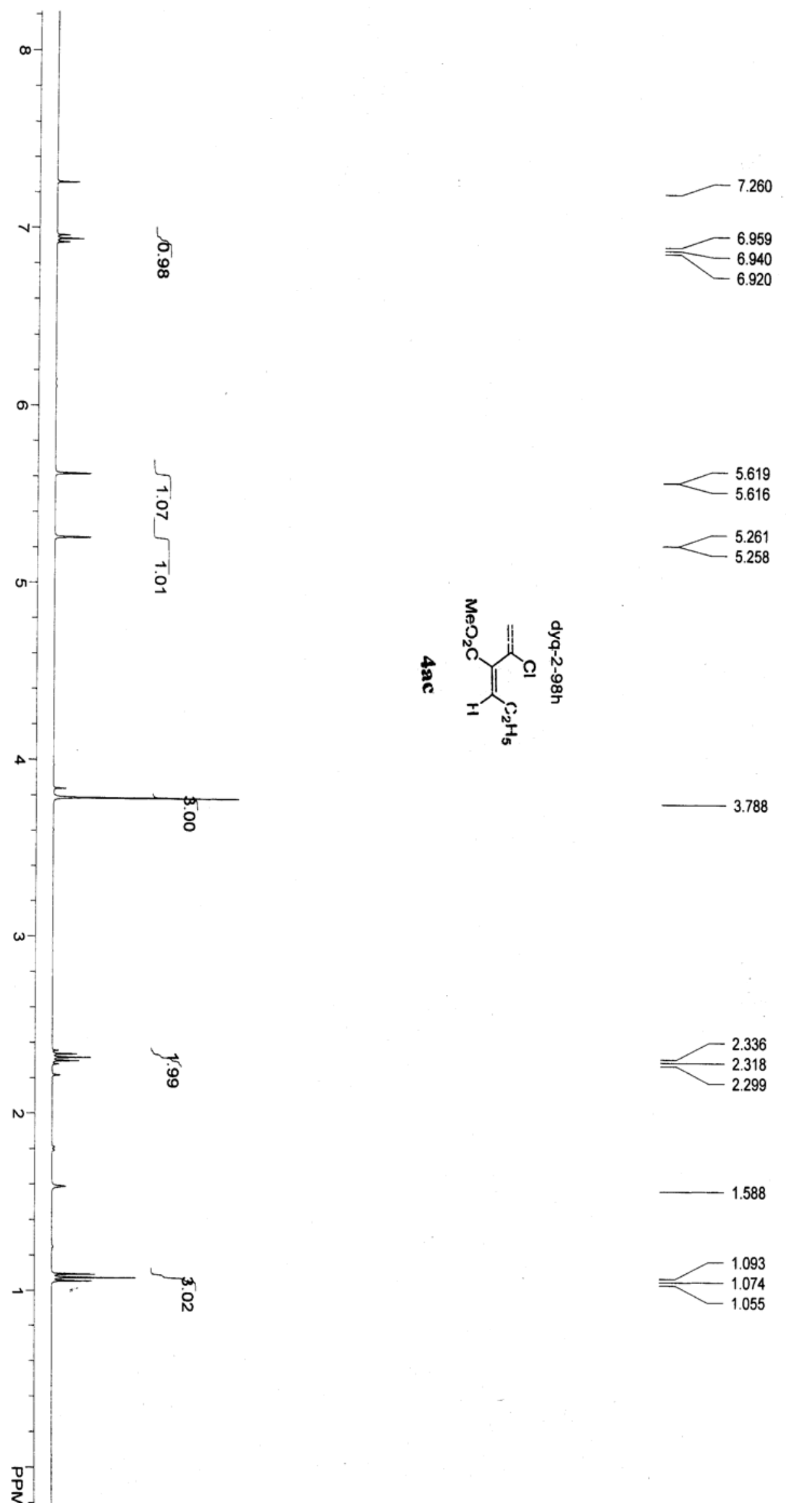

恼
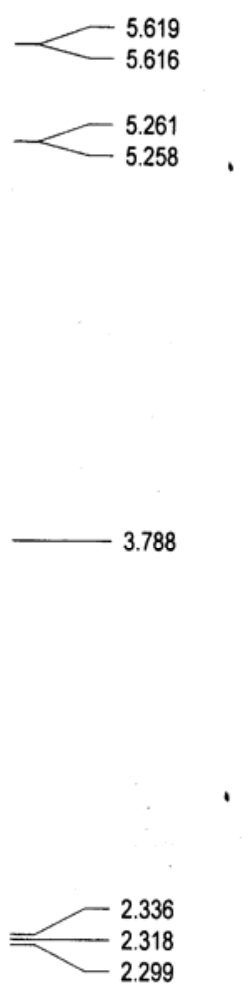

1.588

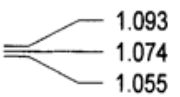



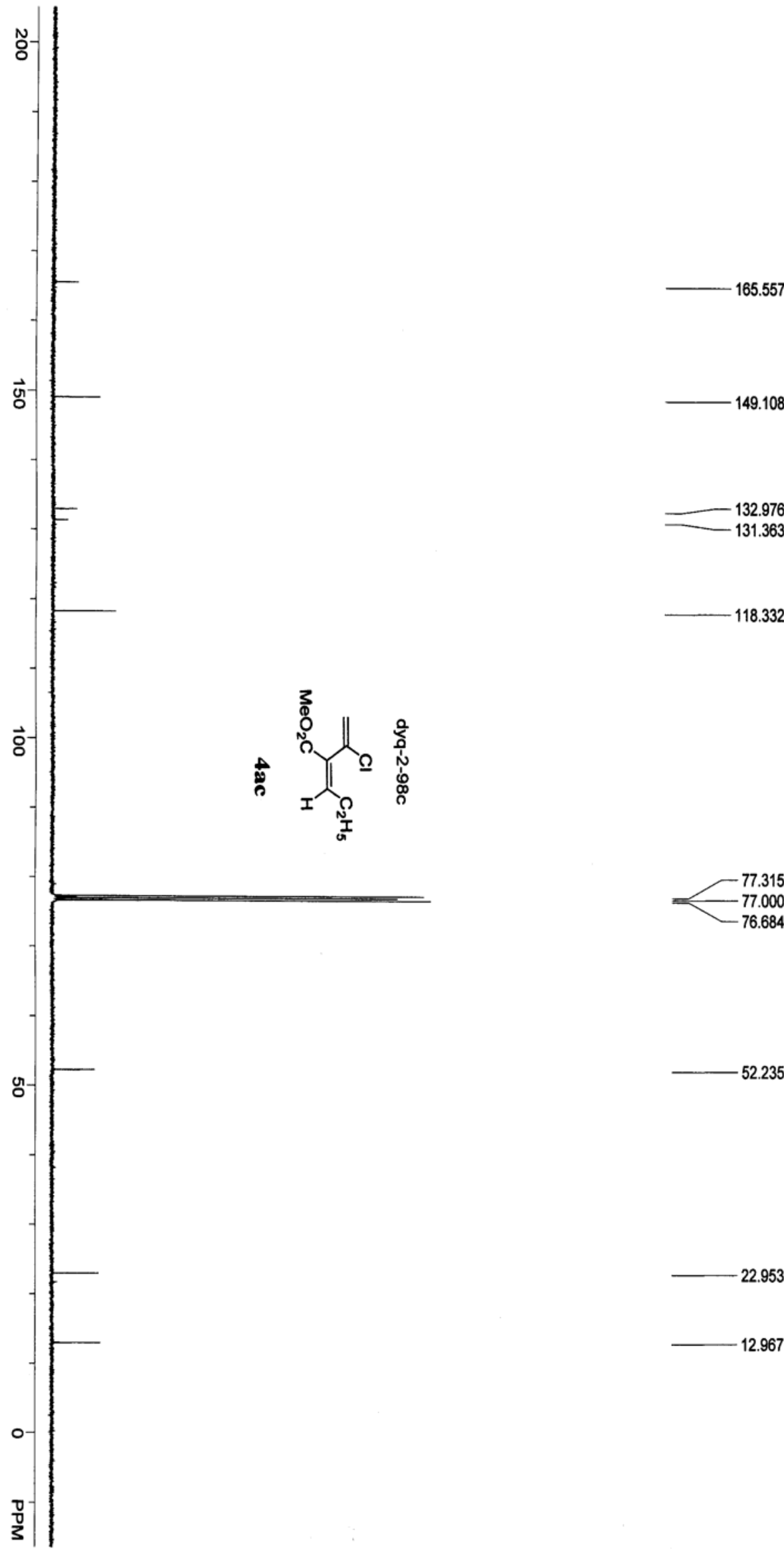

$-118.332$
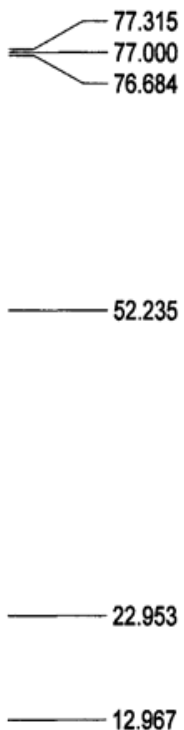

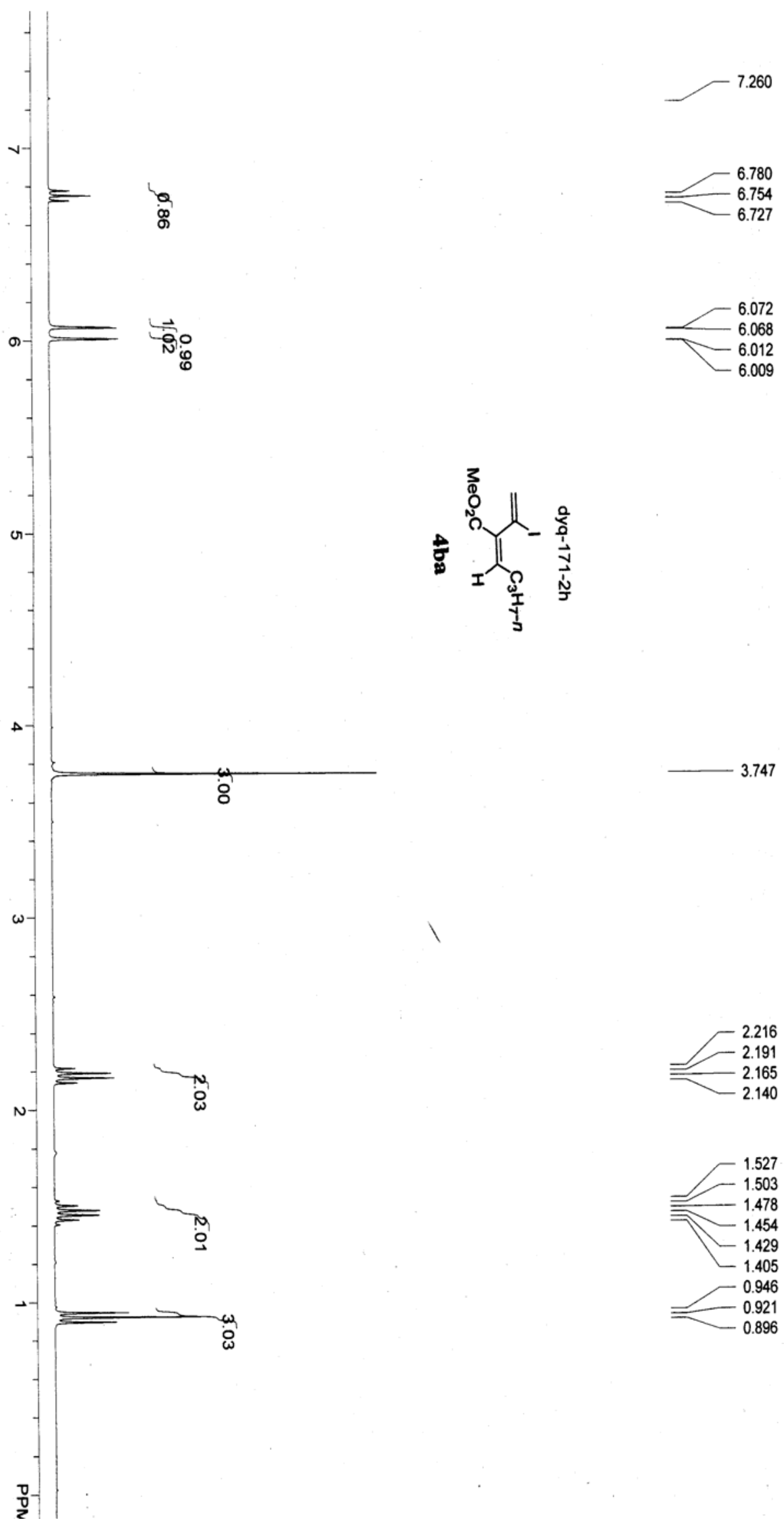


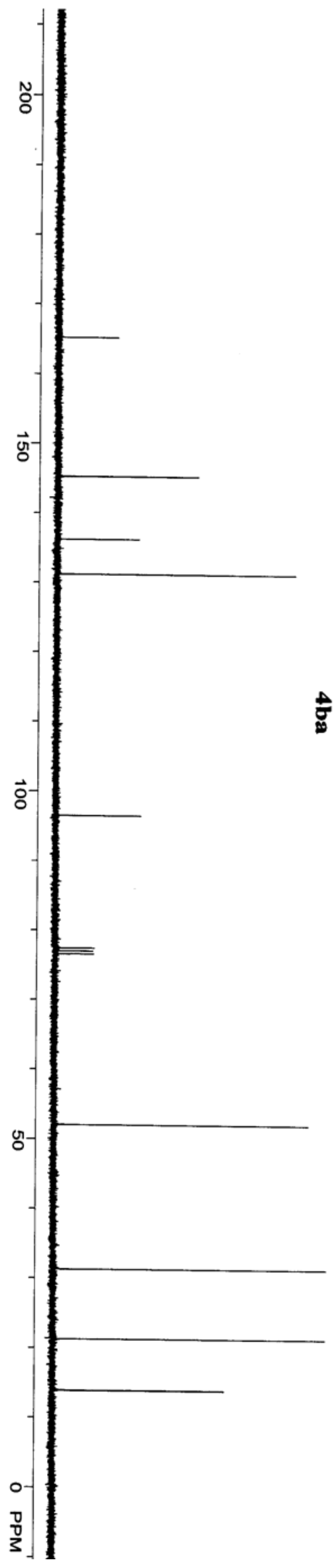

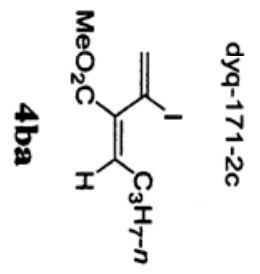
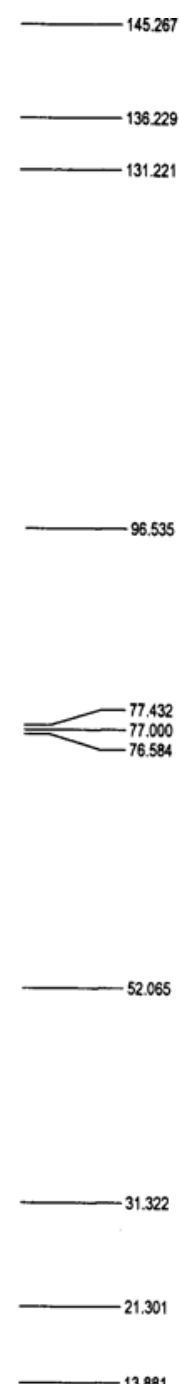

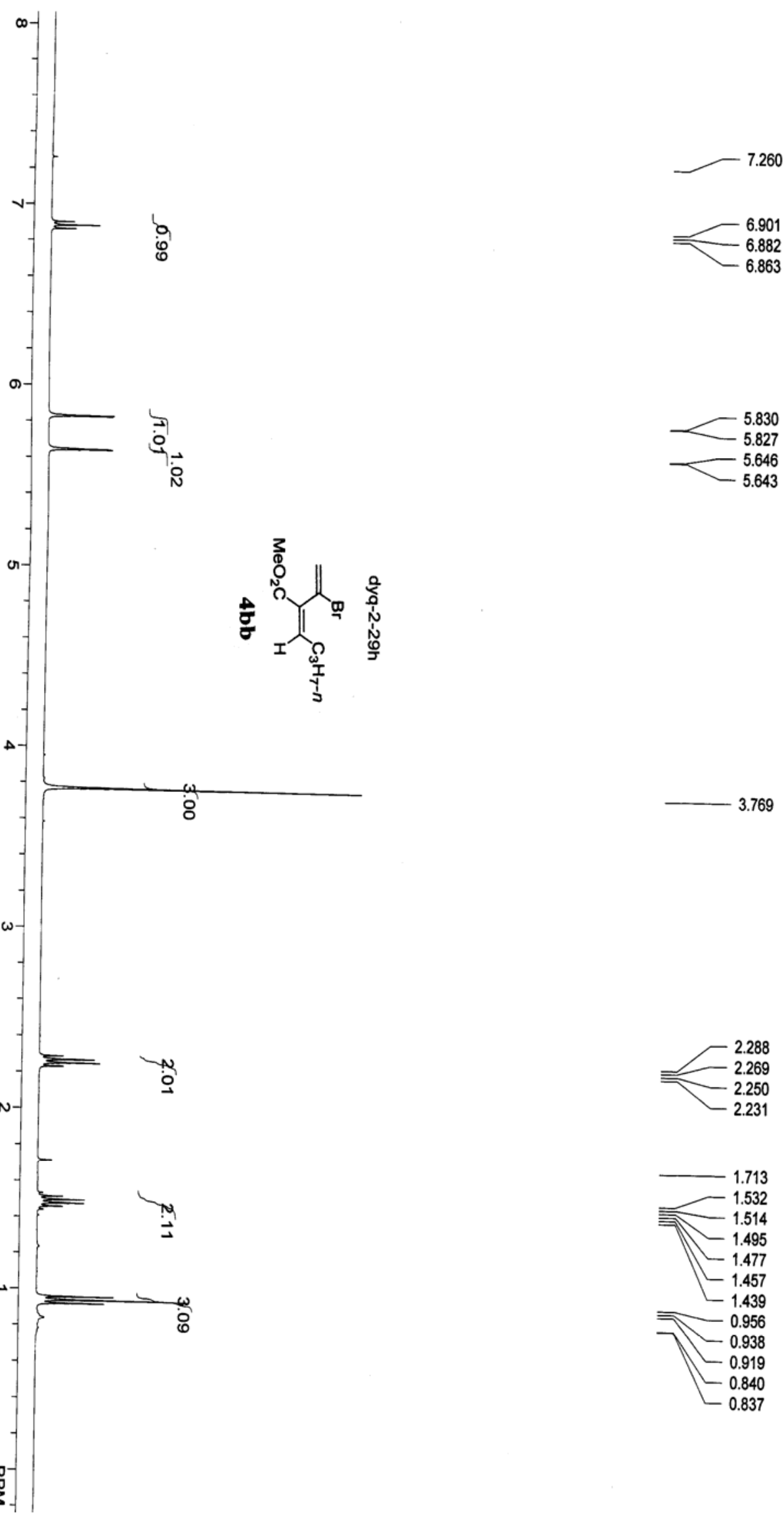


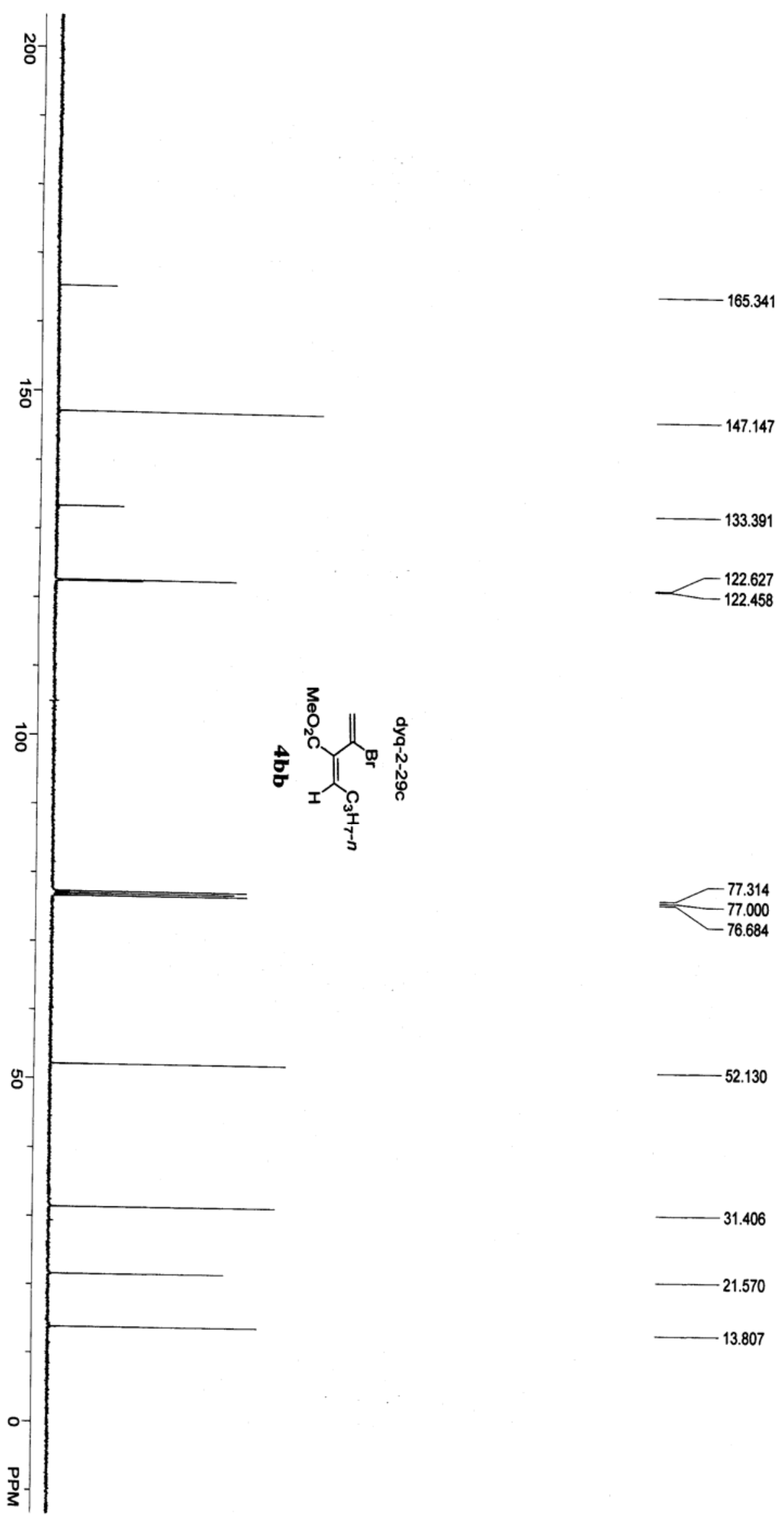



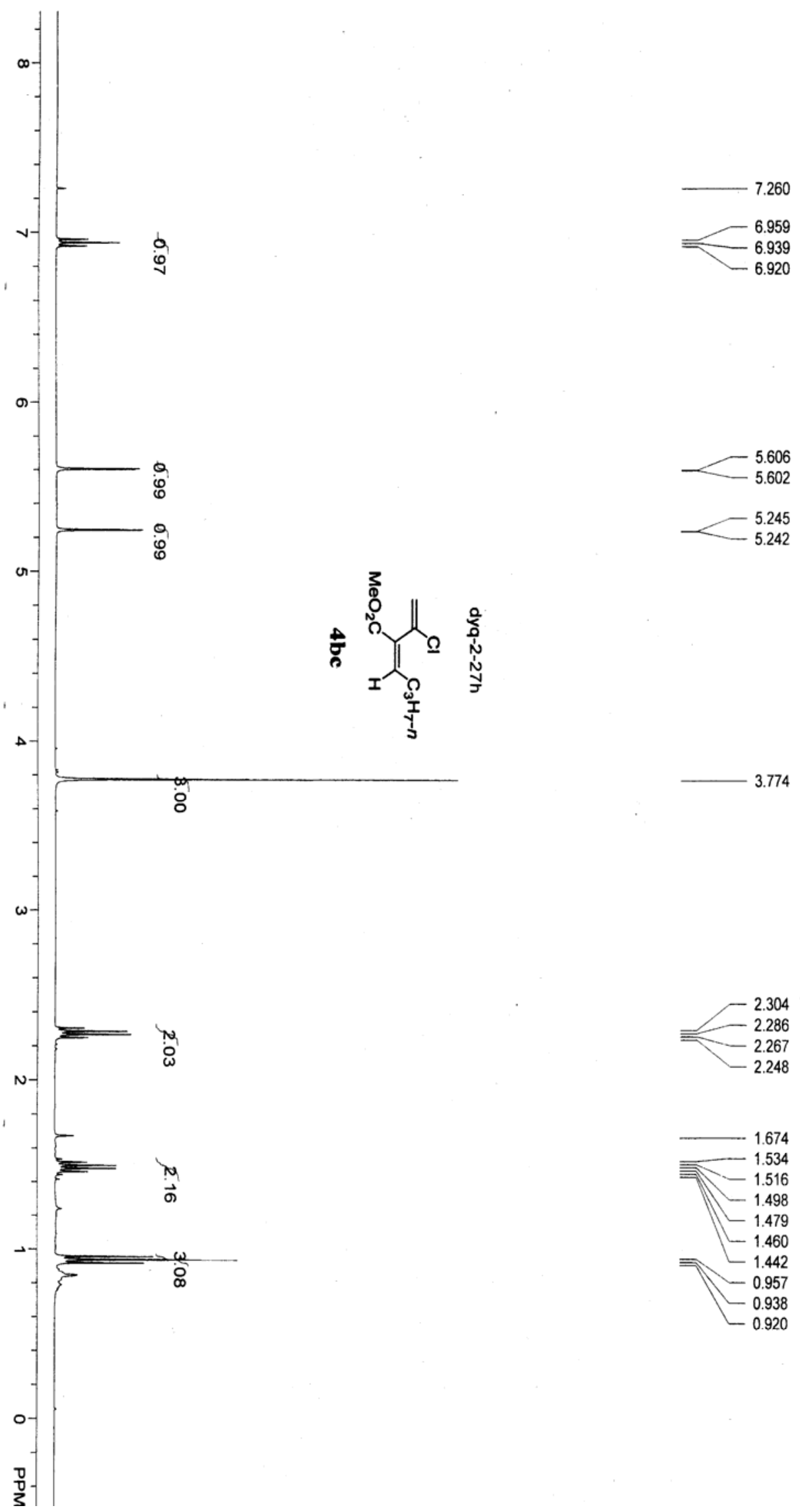


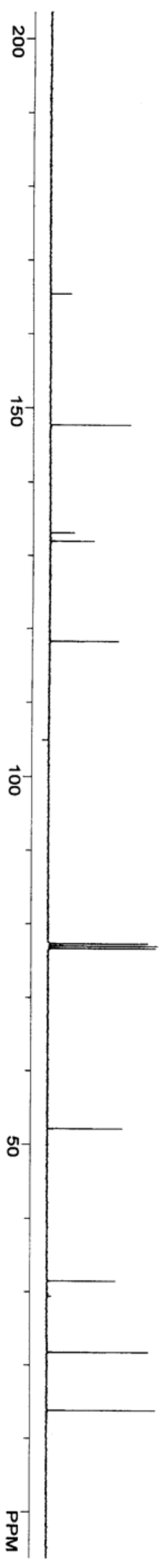

165.453
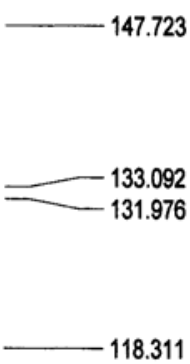

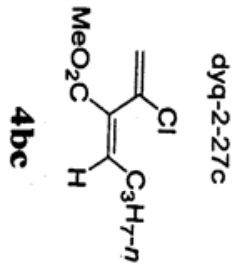
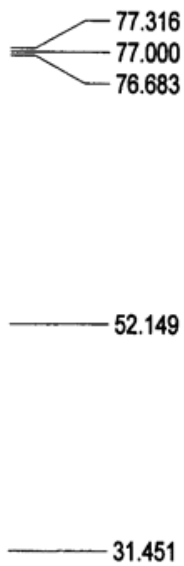

$-21.708$

13.797 

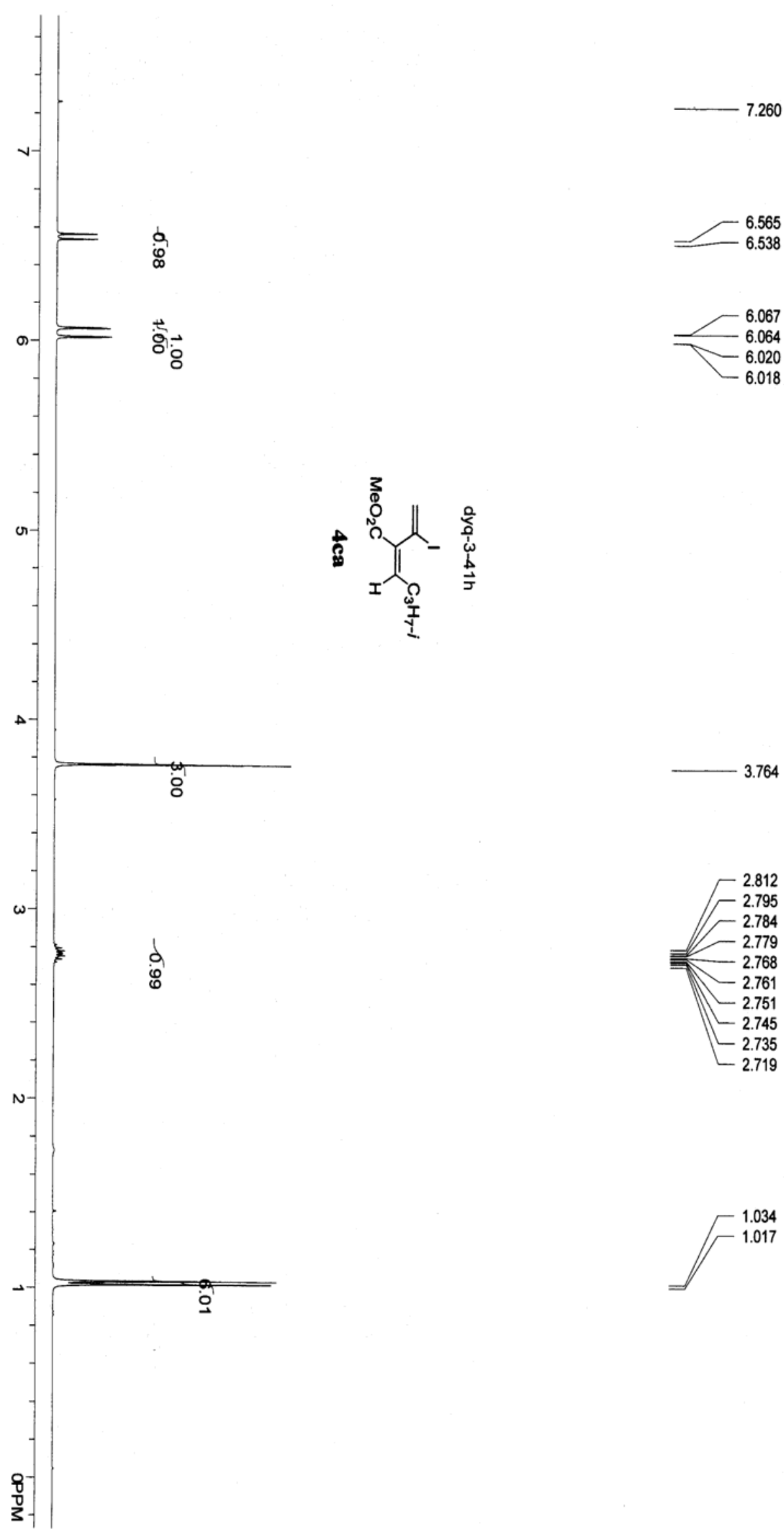

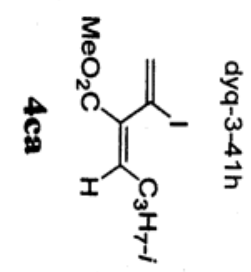
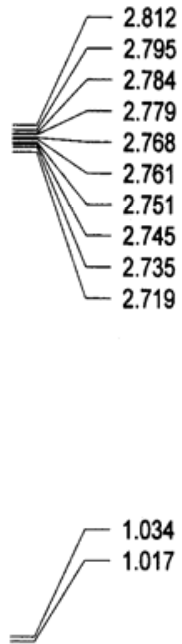

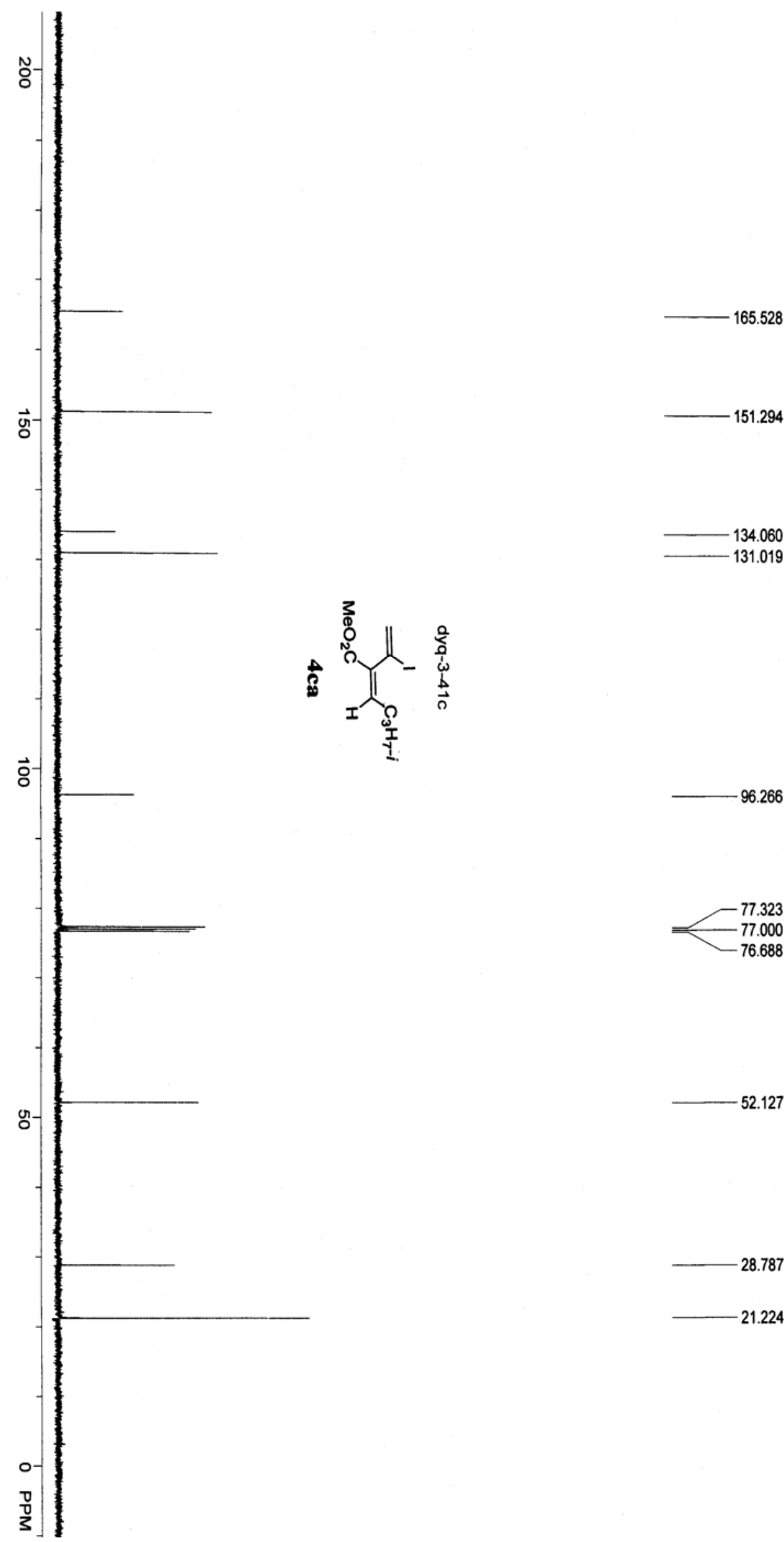

$\overbrace{}^{1}$

134.060

$-131.019$ 

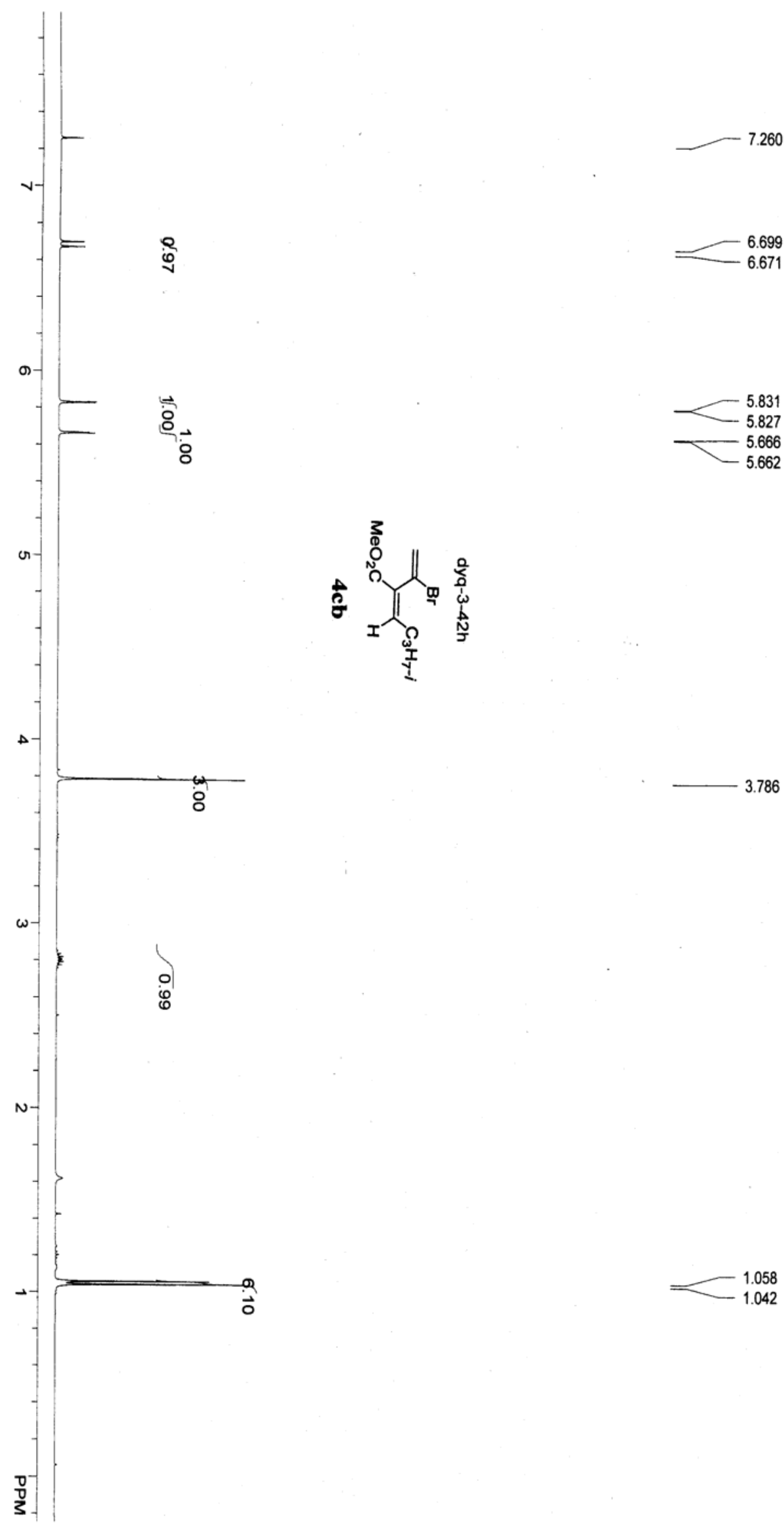


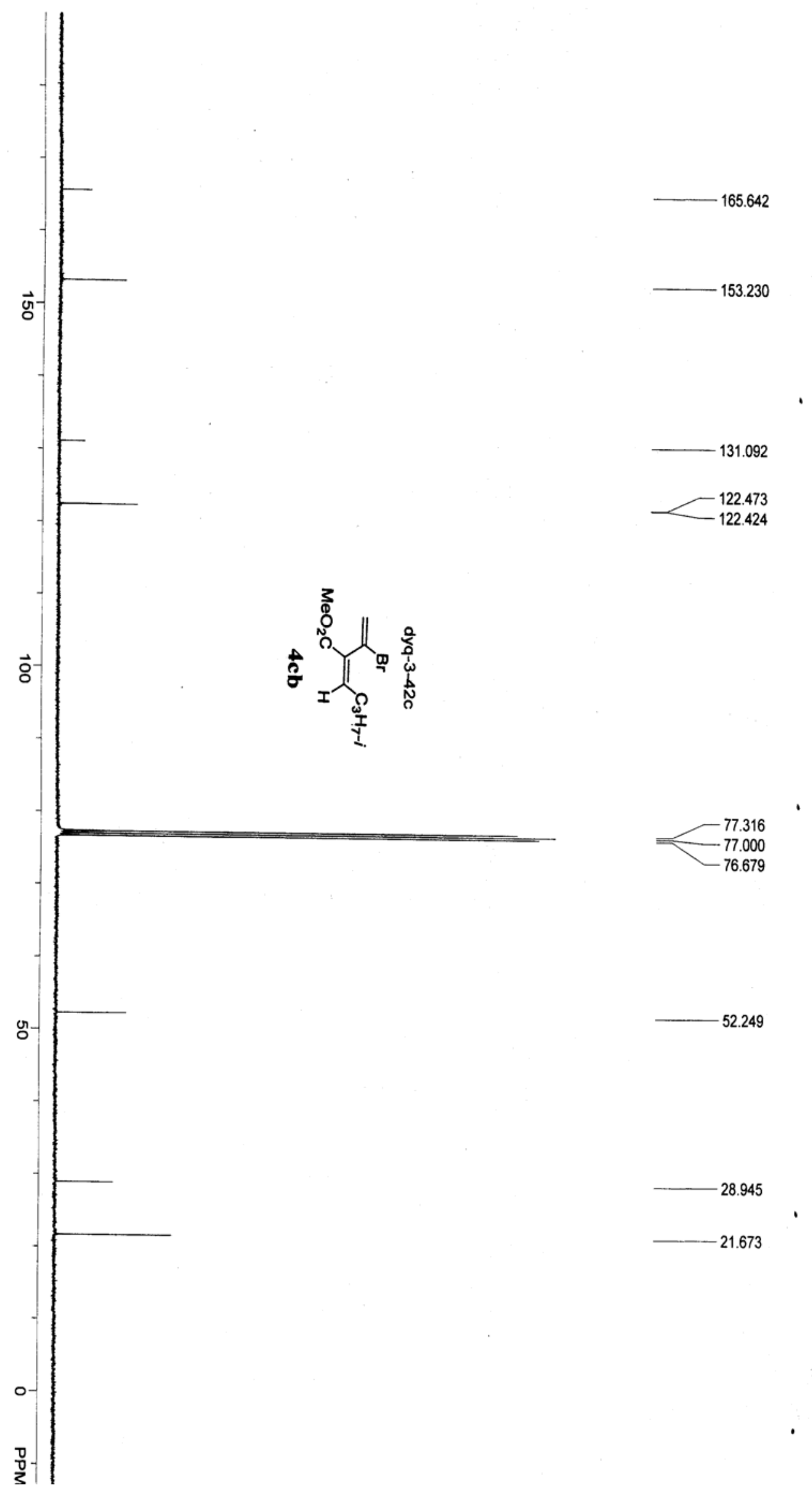



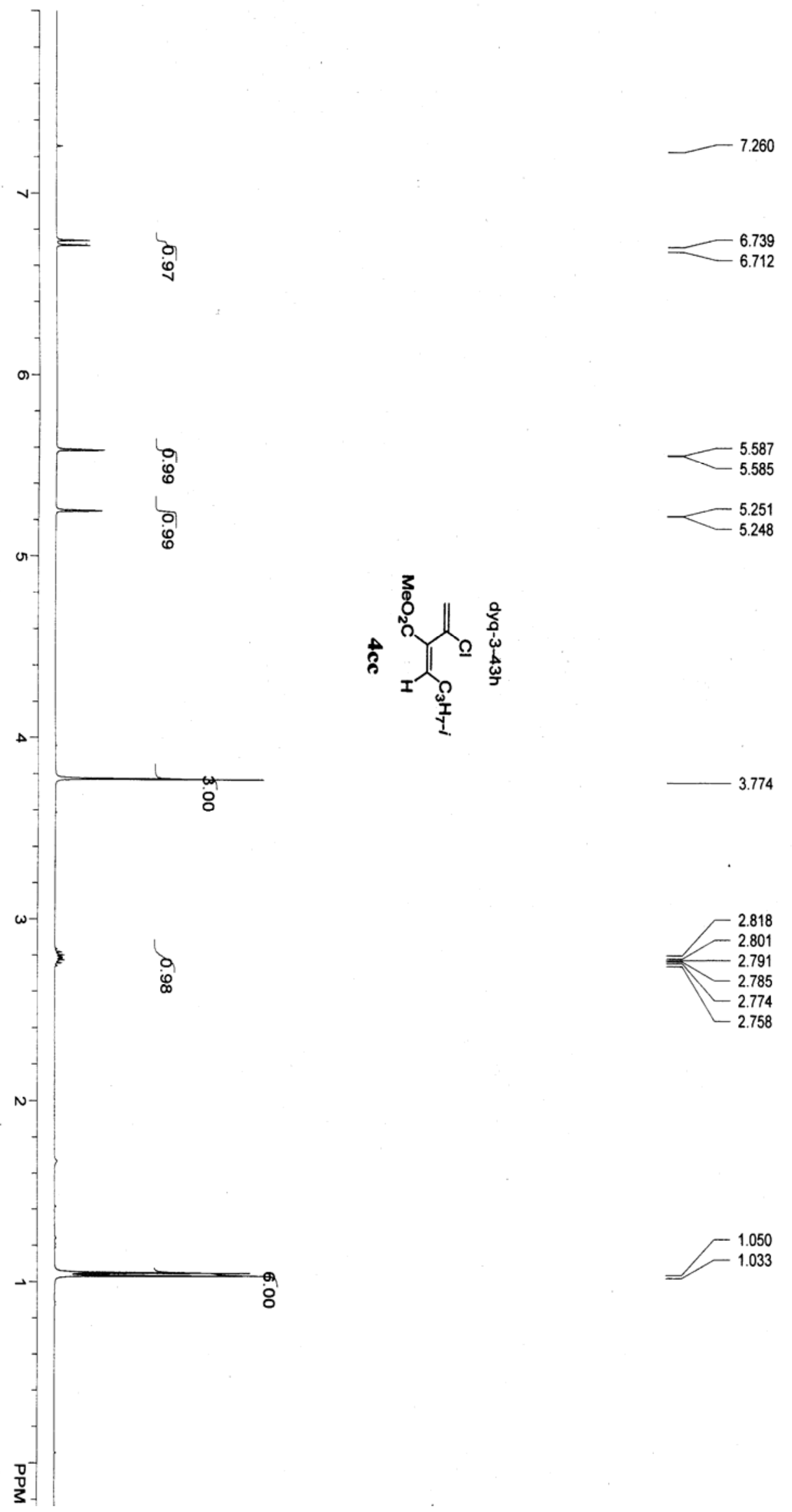

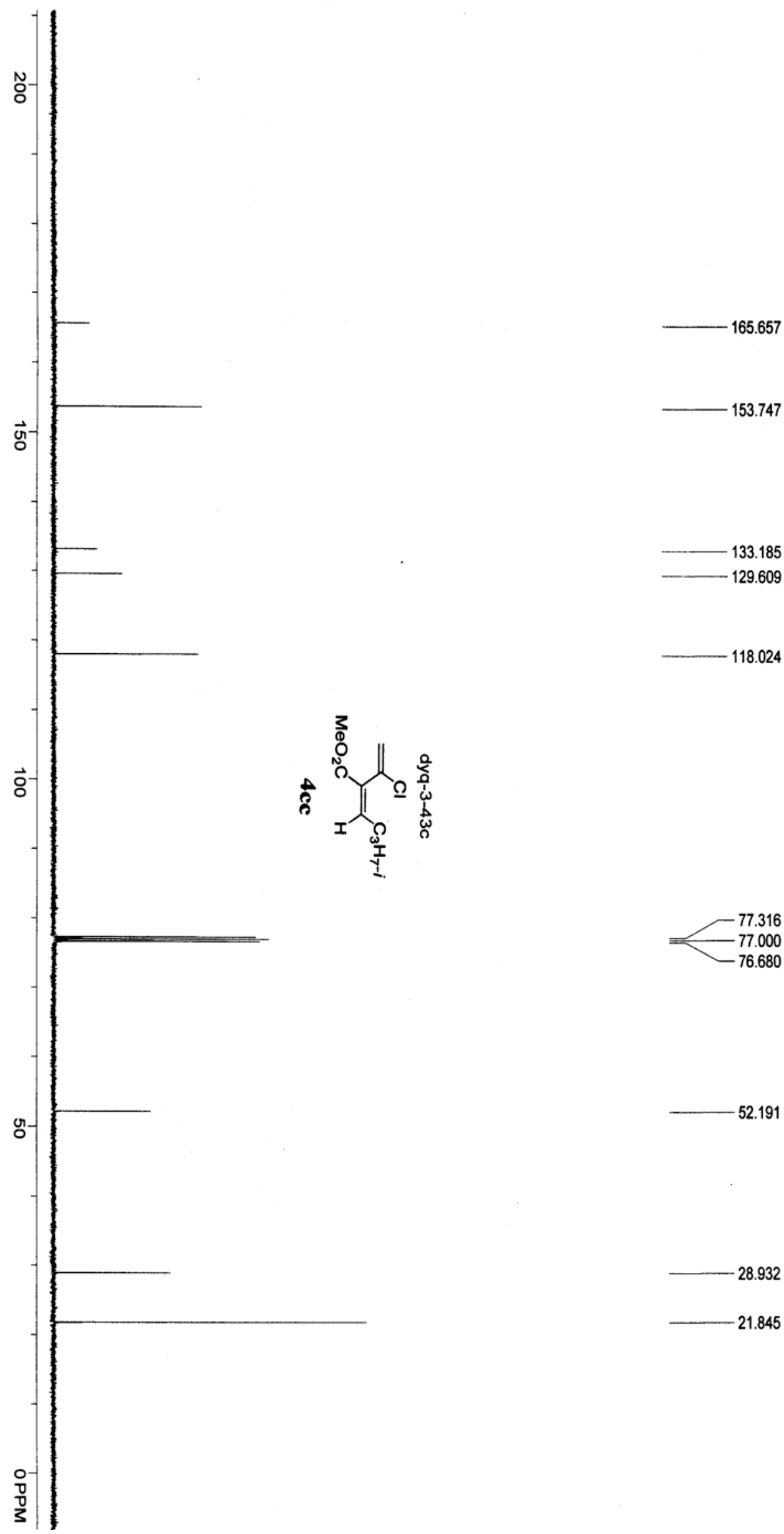

133.185

129.609

118.024
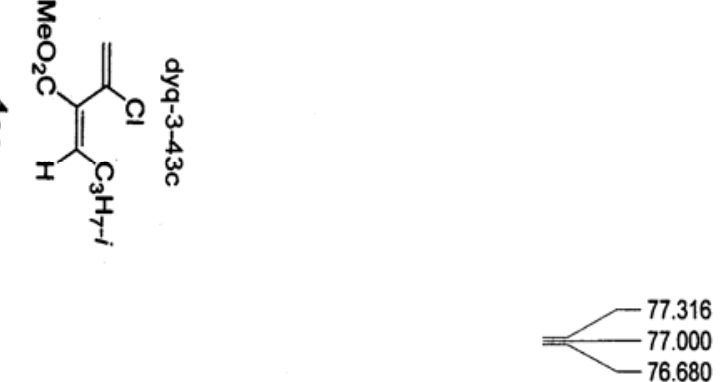

52.191 


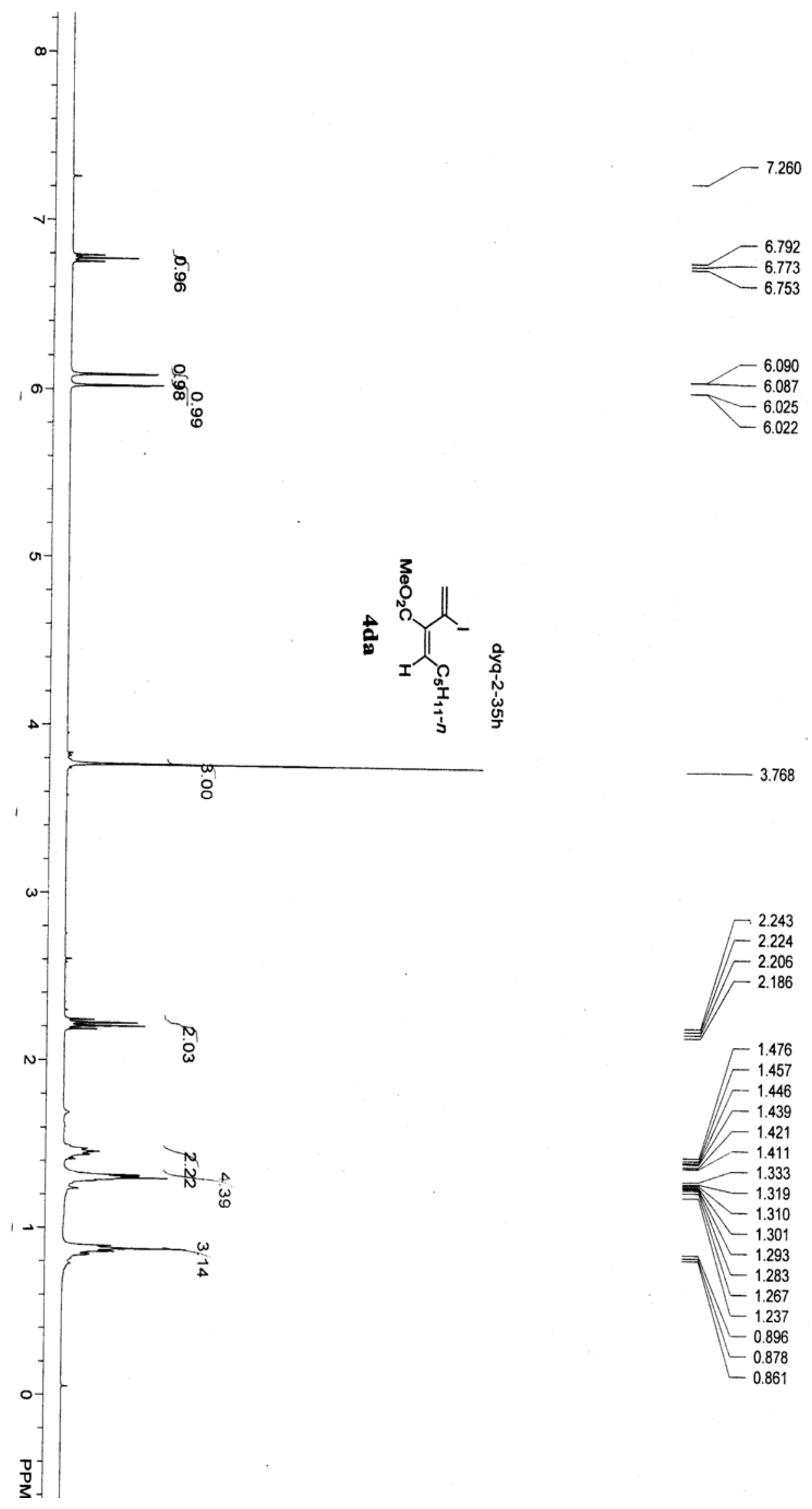




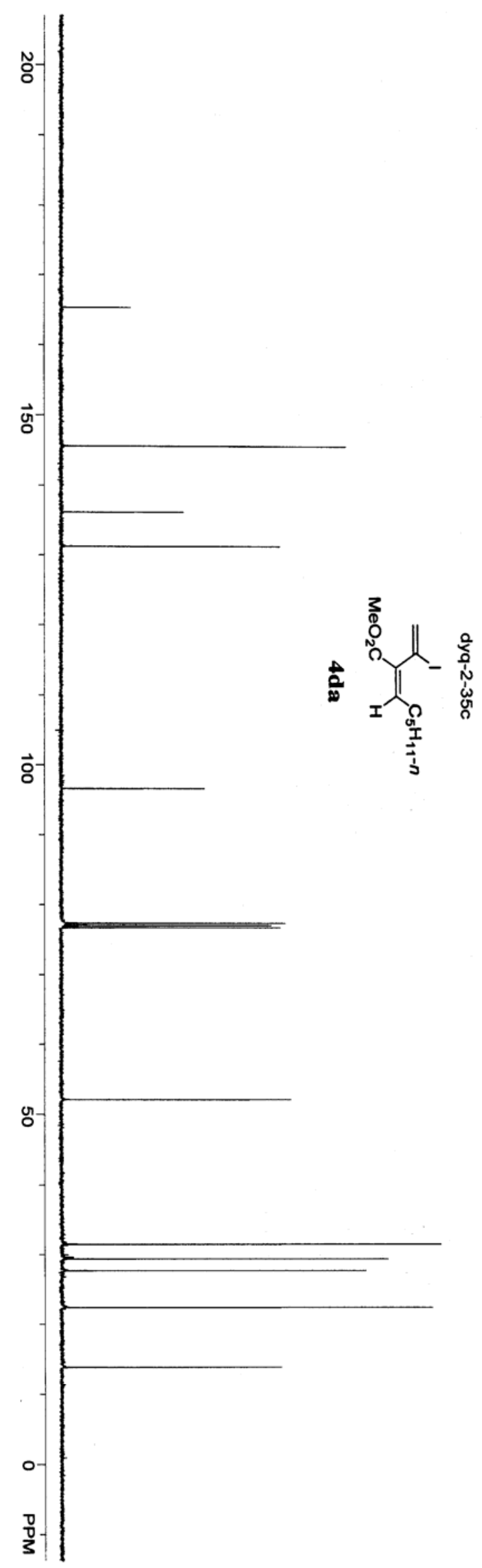

165.349

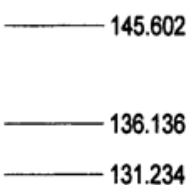

96.586
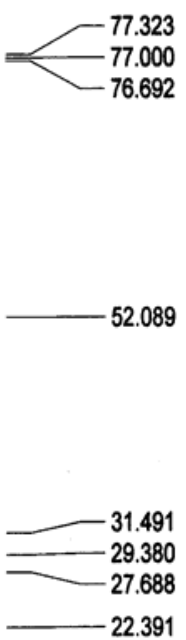

13.859 

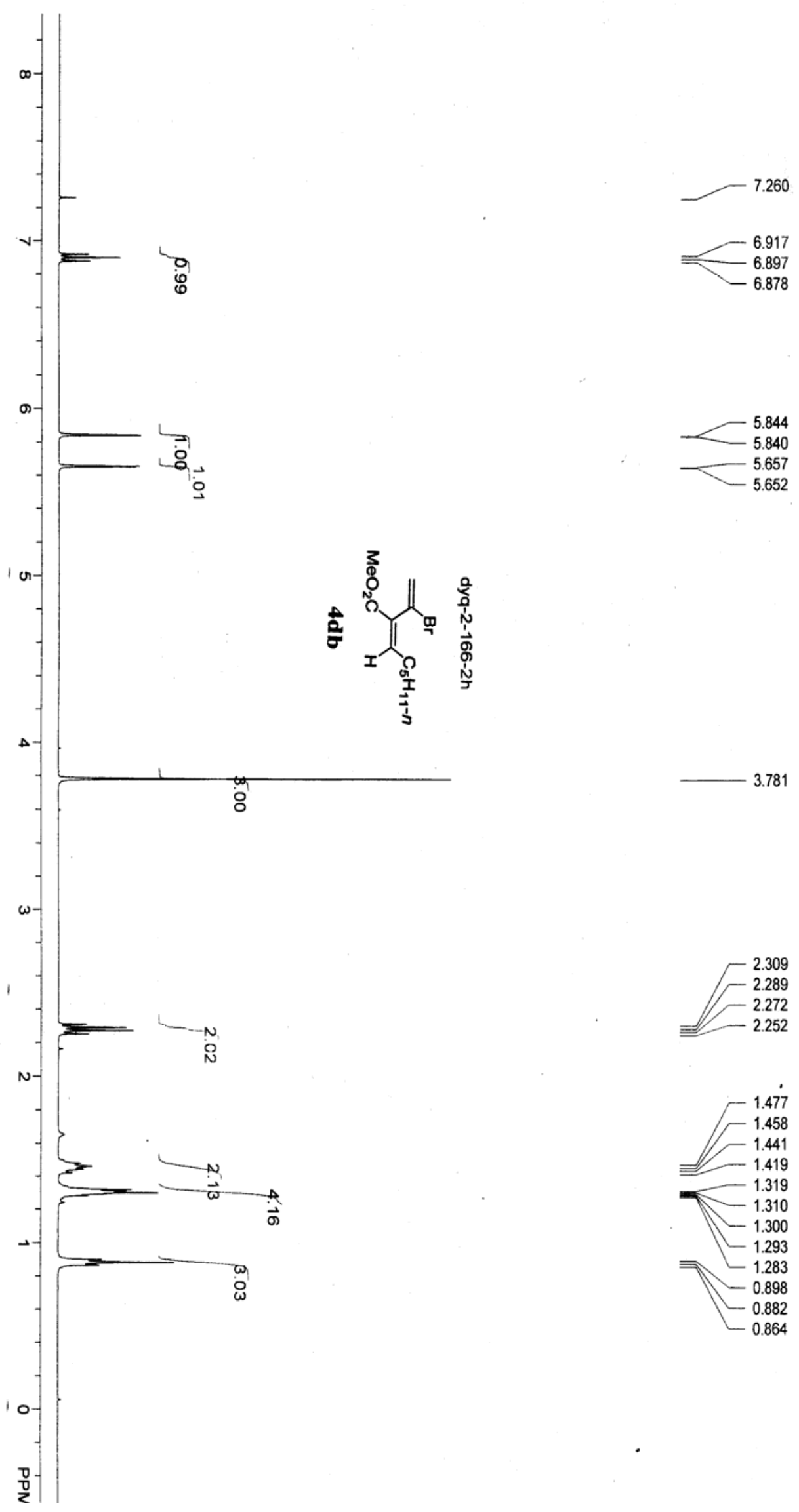

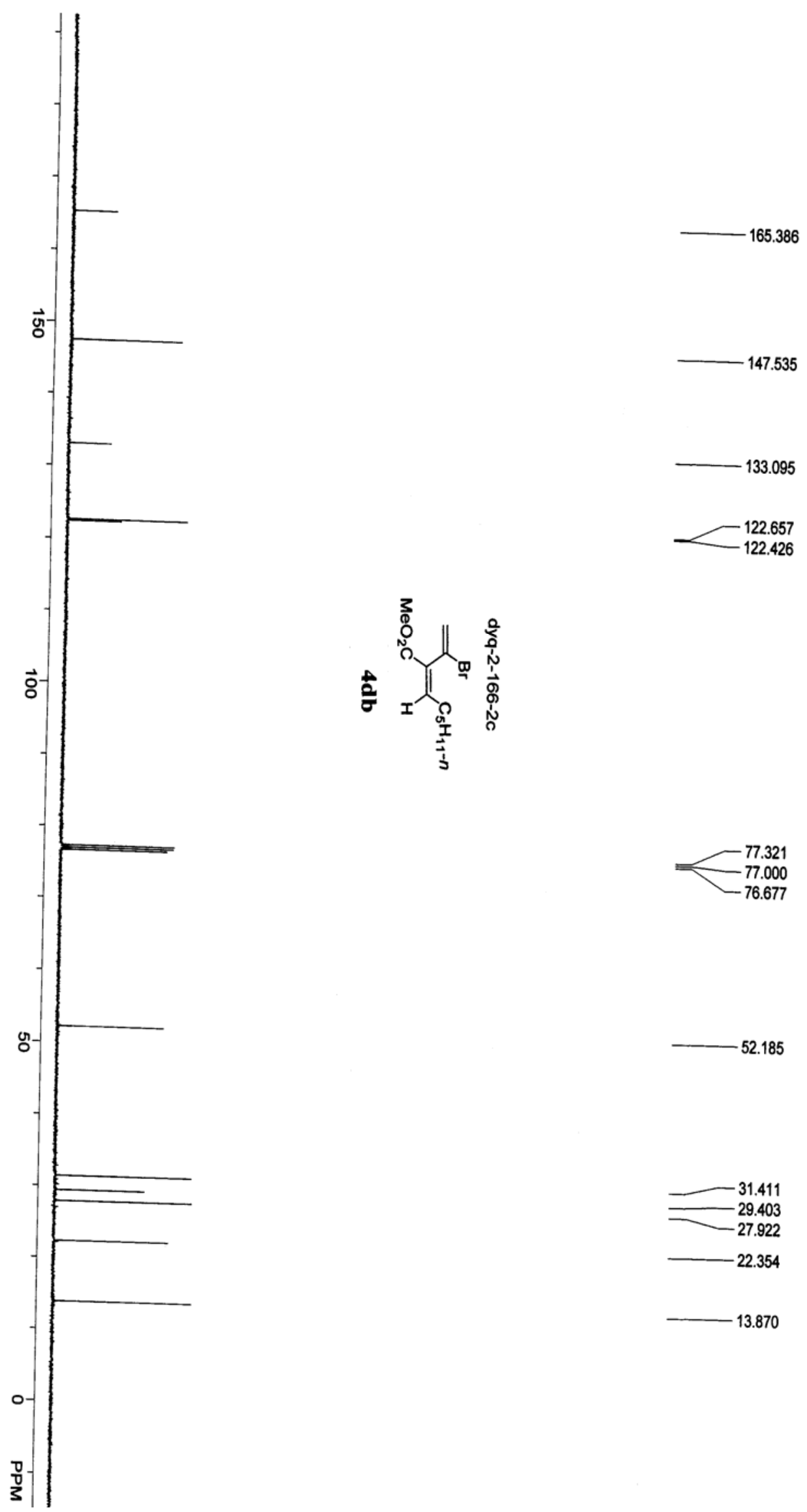

52.185

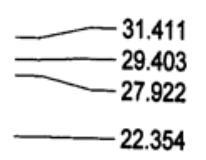

$-13.870$ 


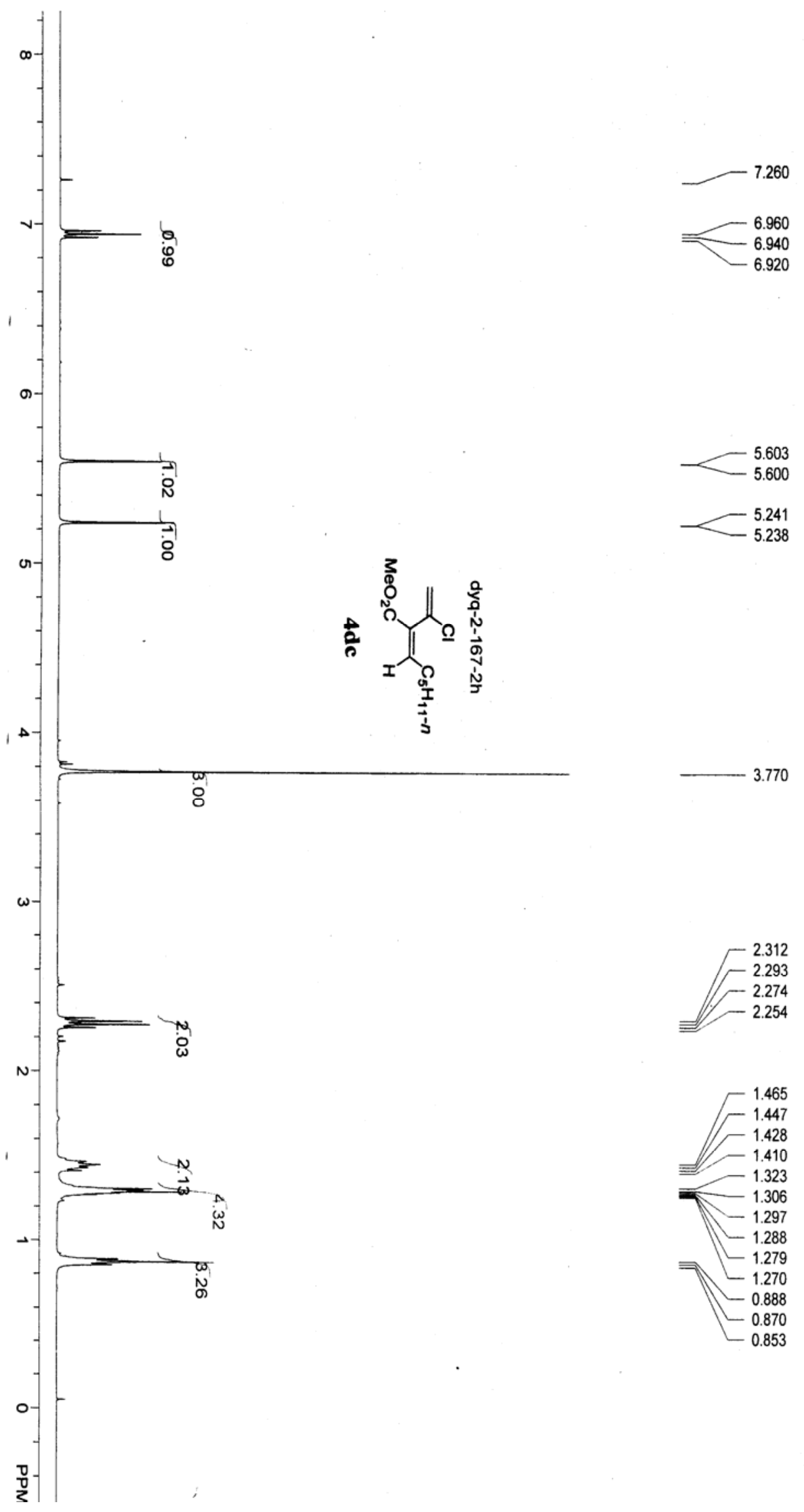



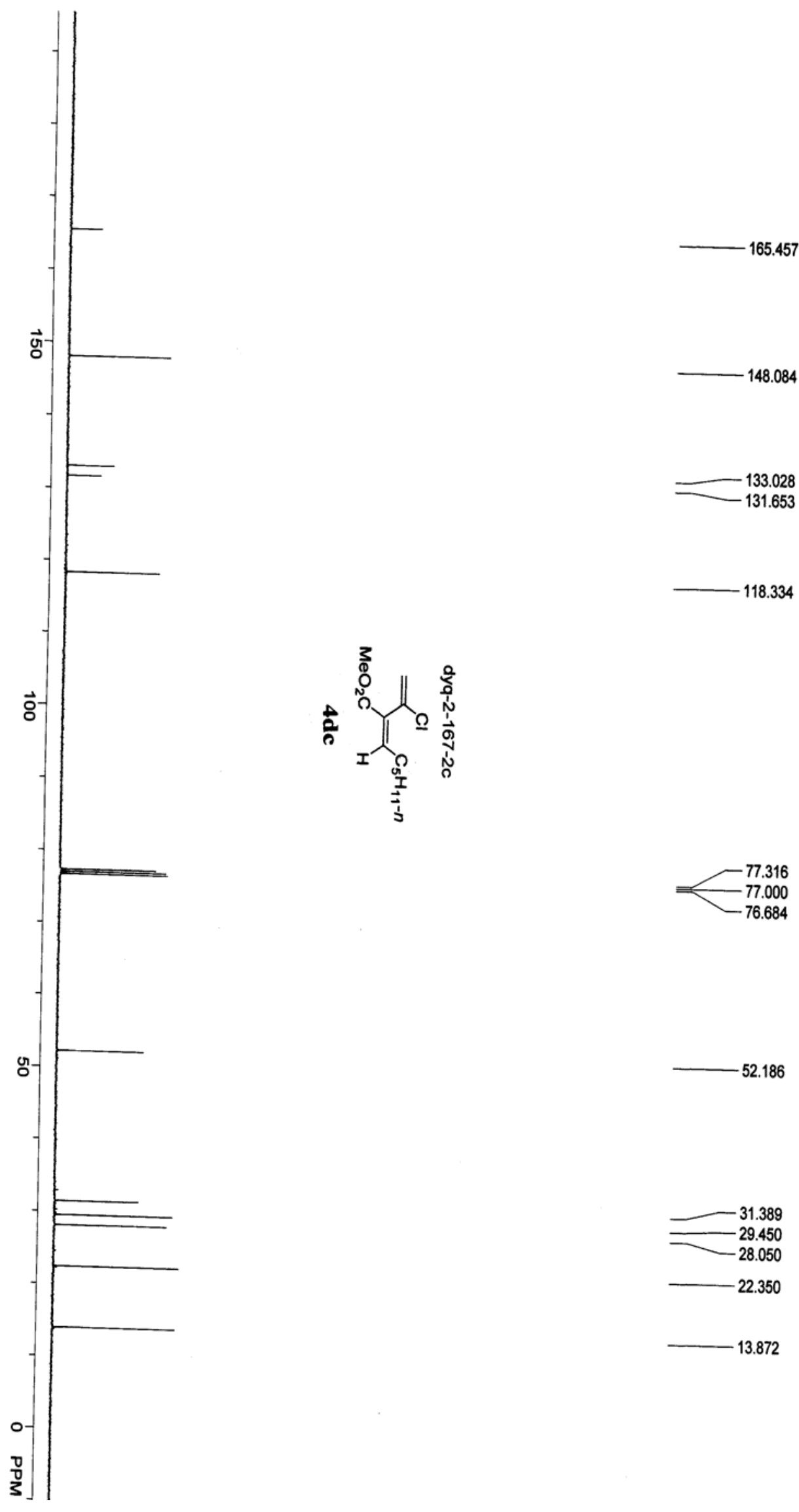

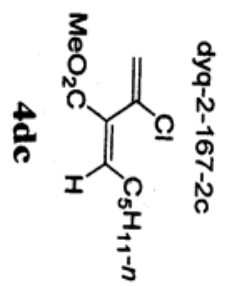

52.186

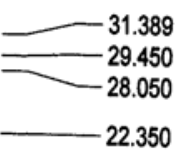

$-13.872$ 

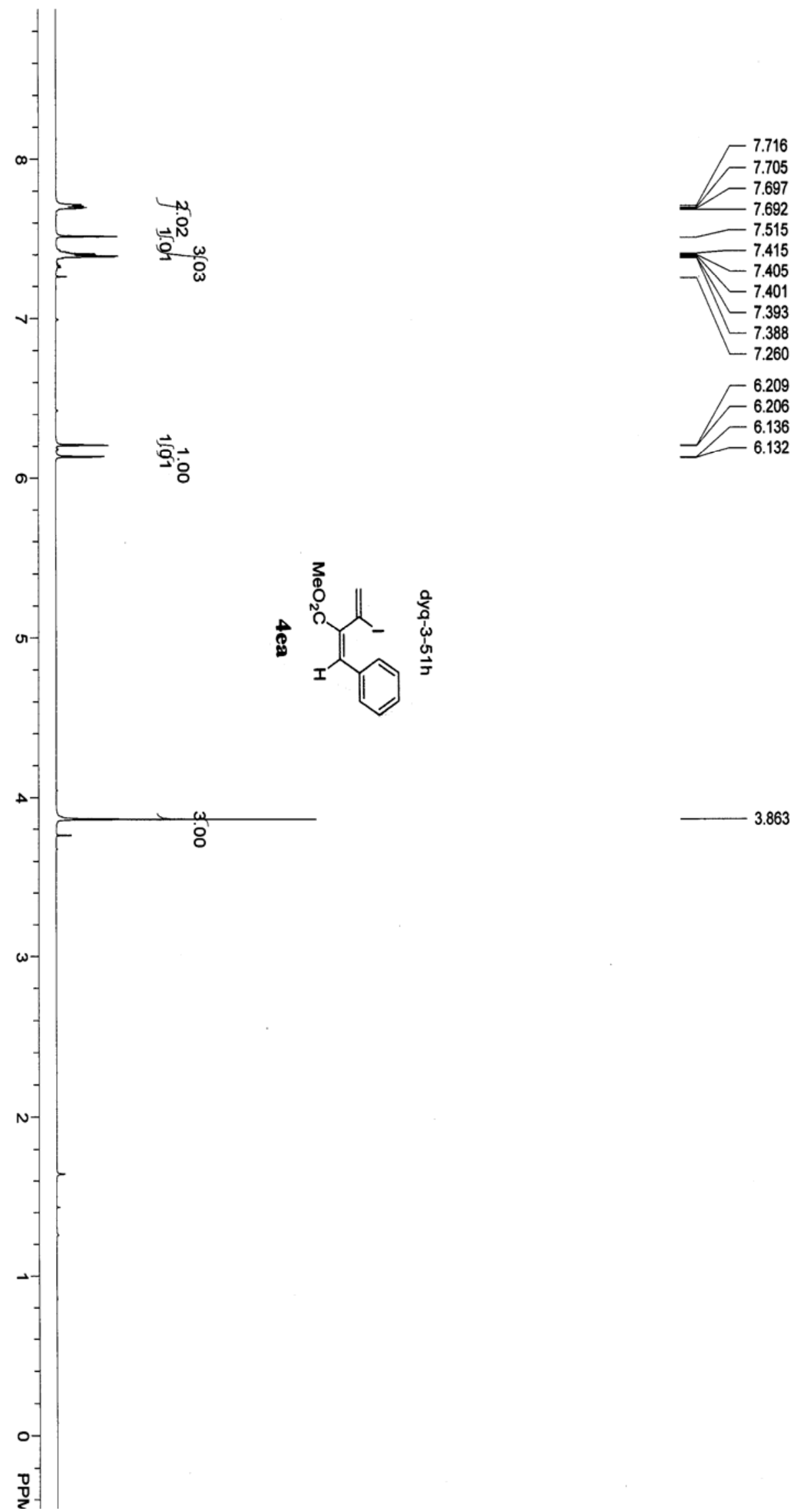


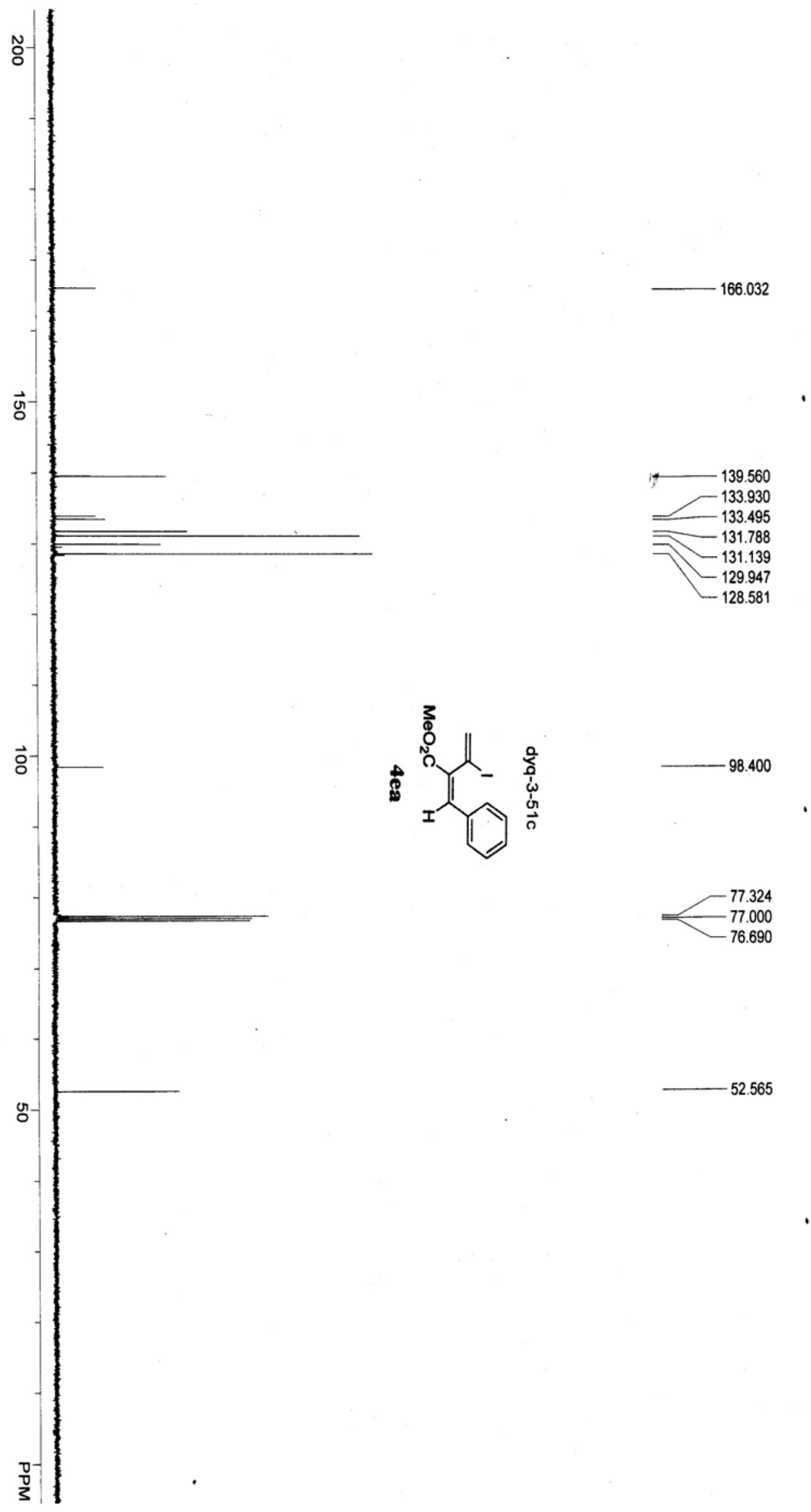



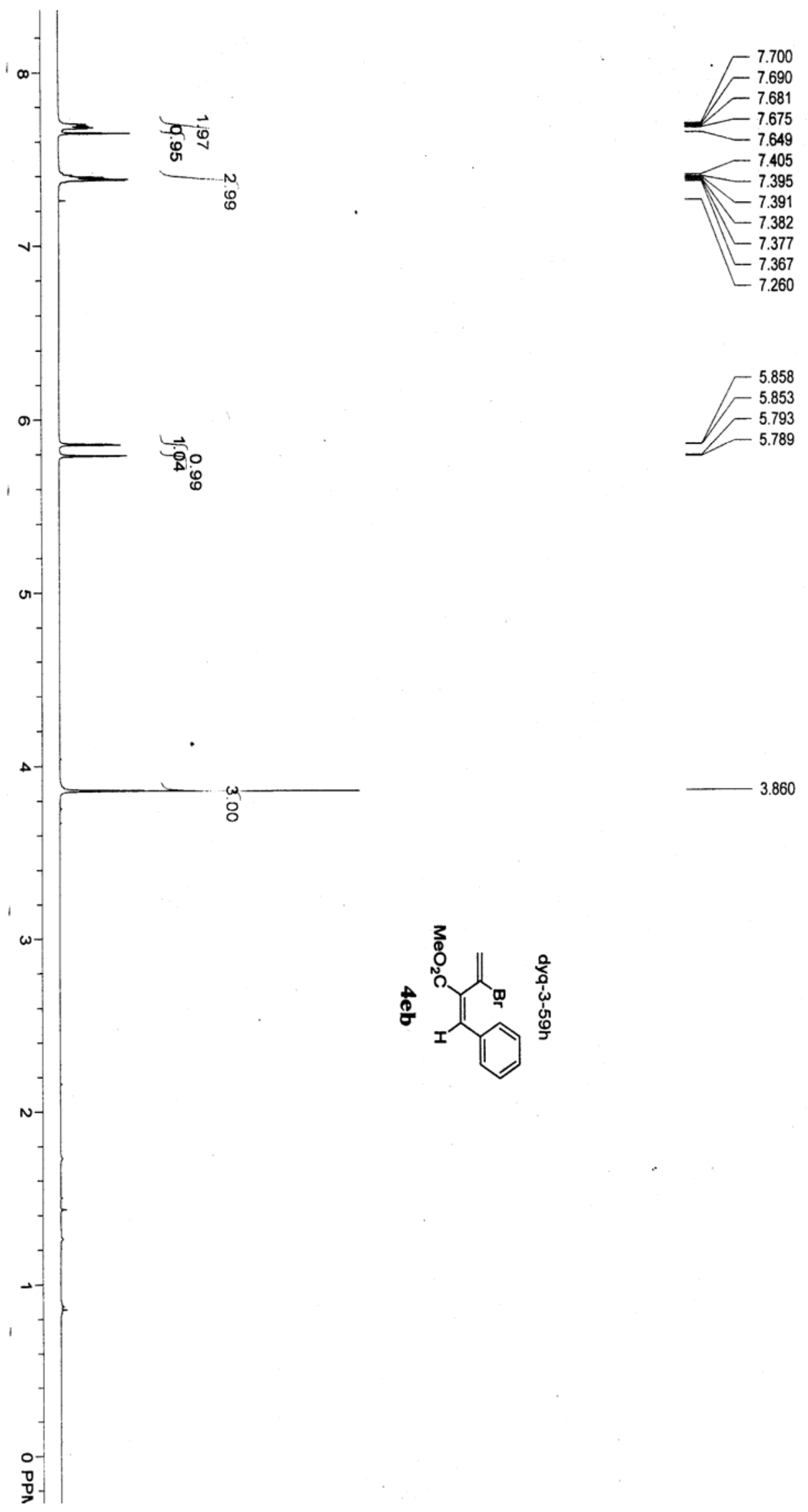


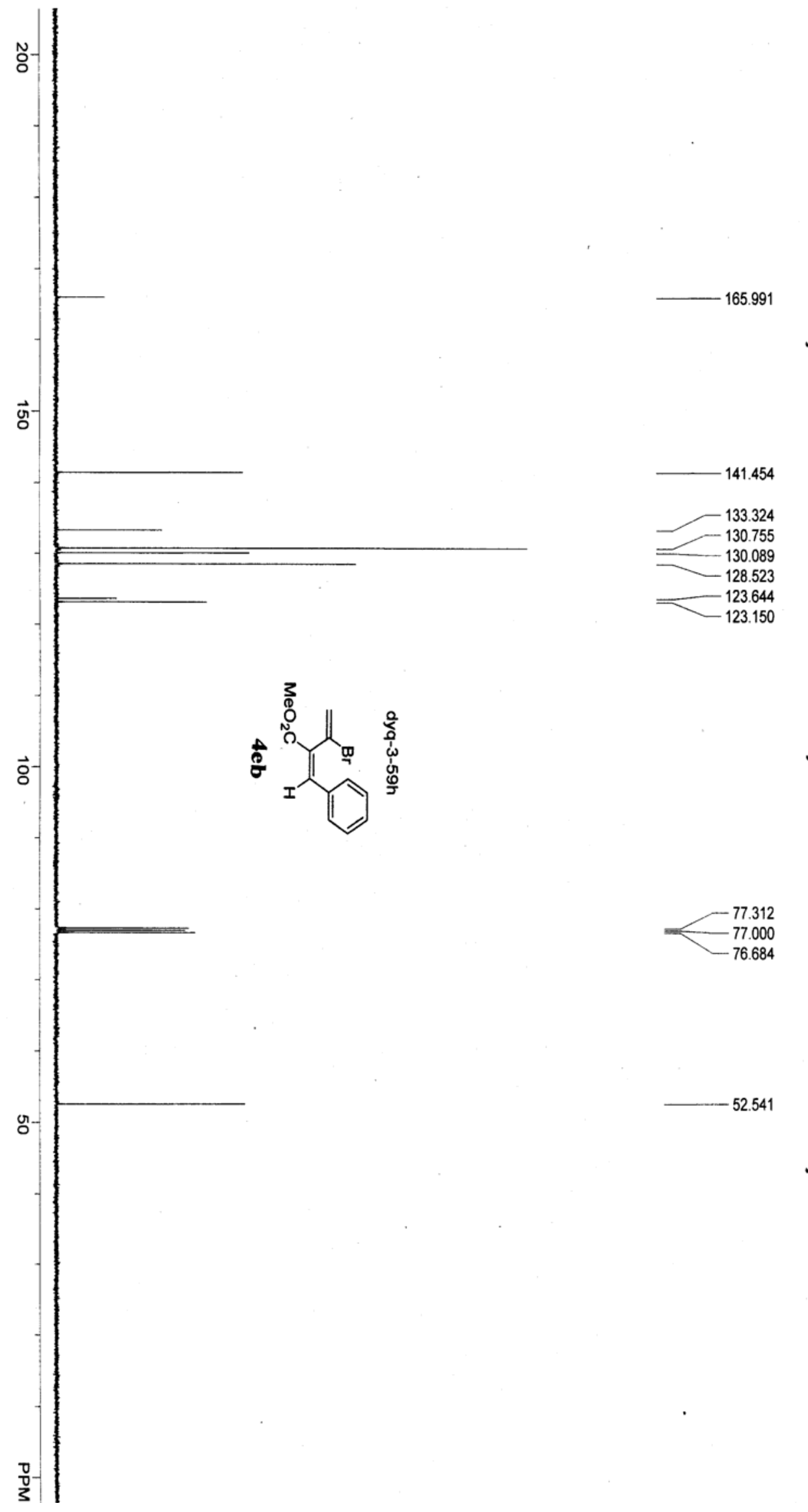



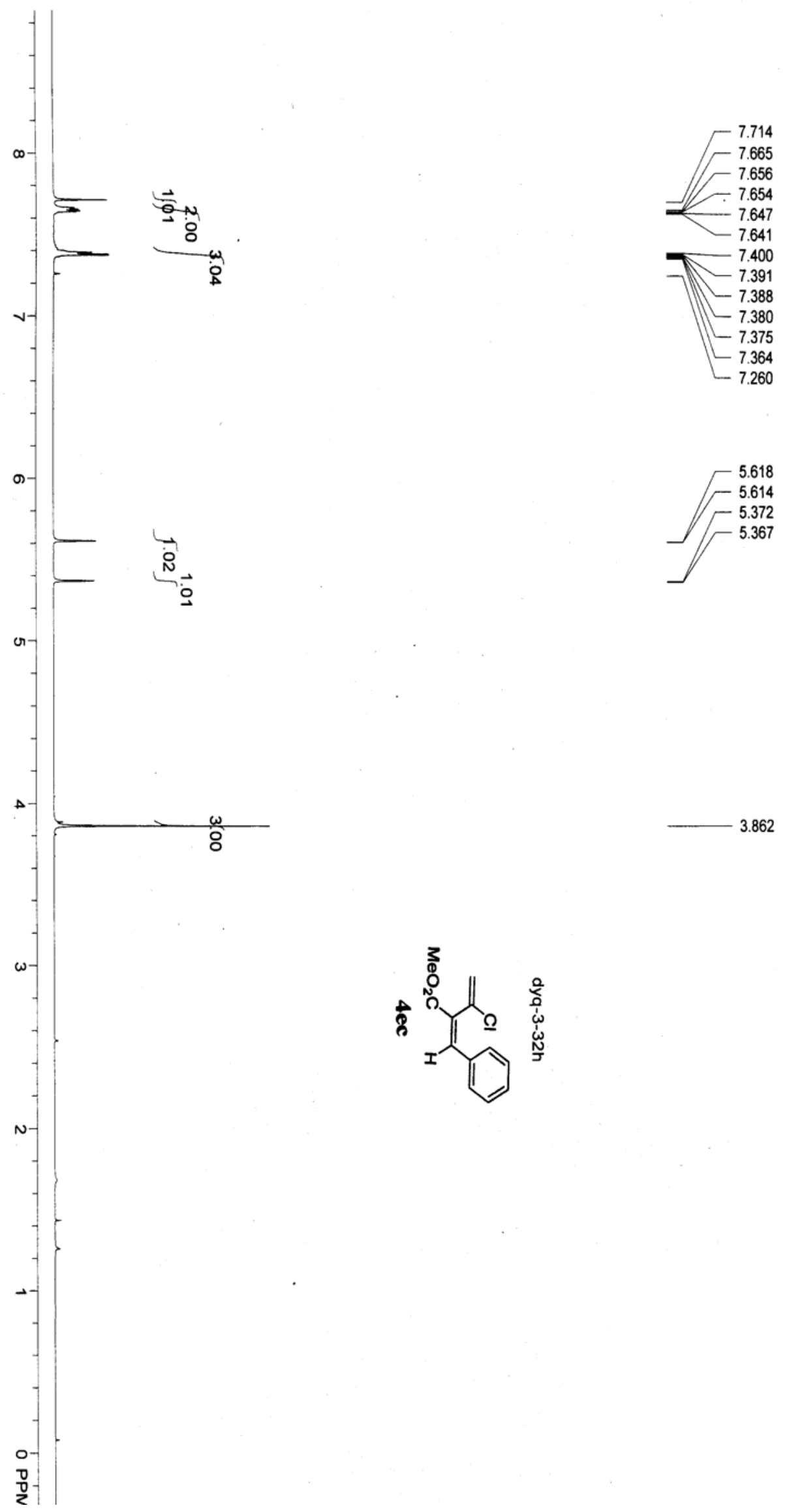

(1) 


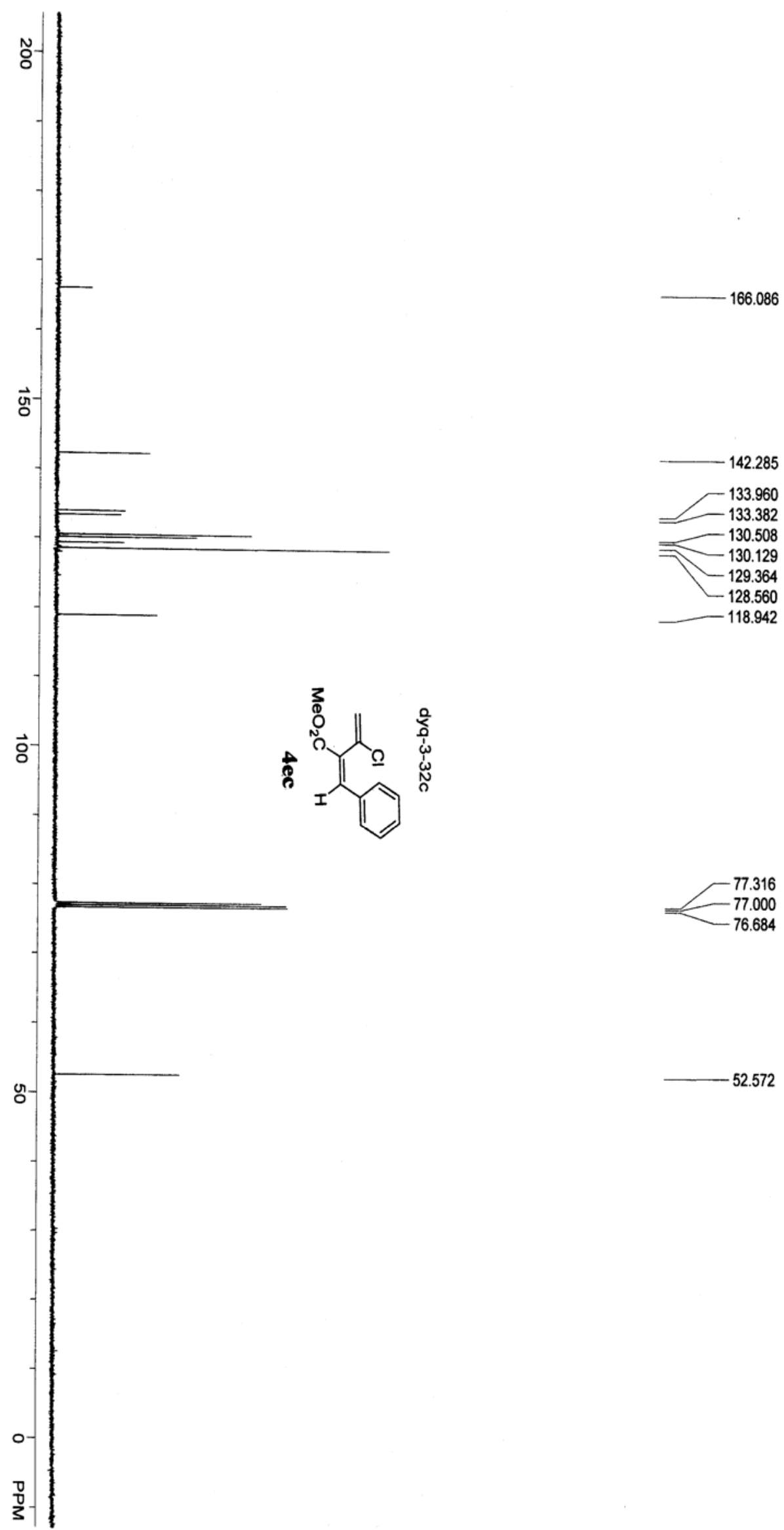



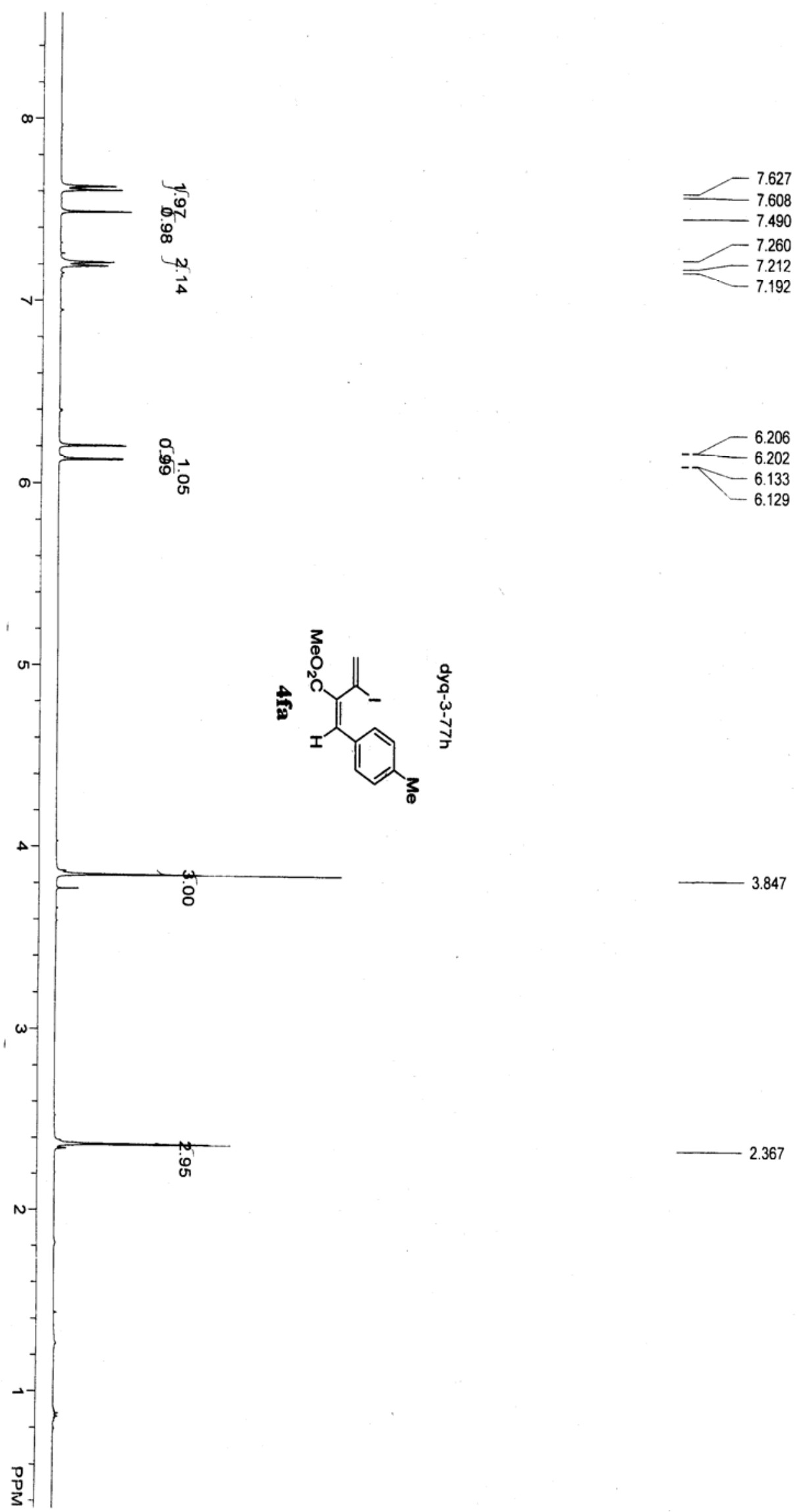

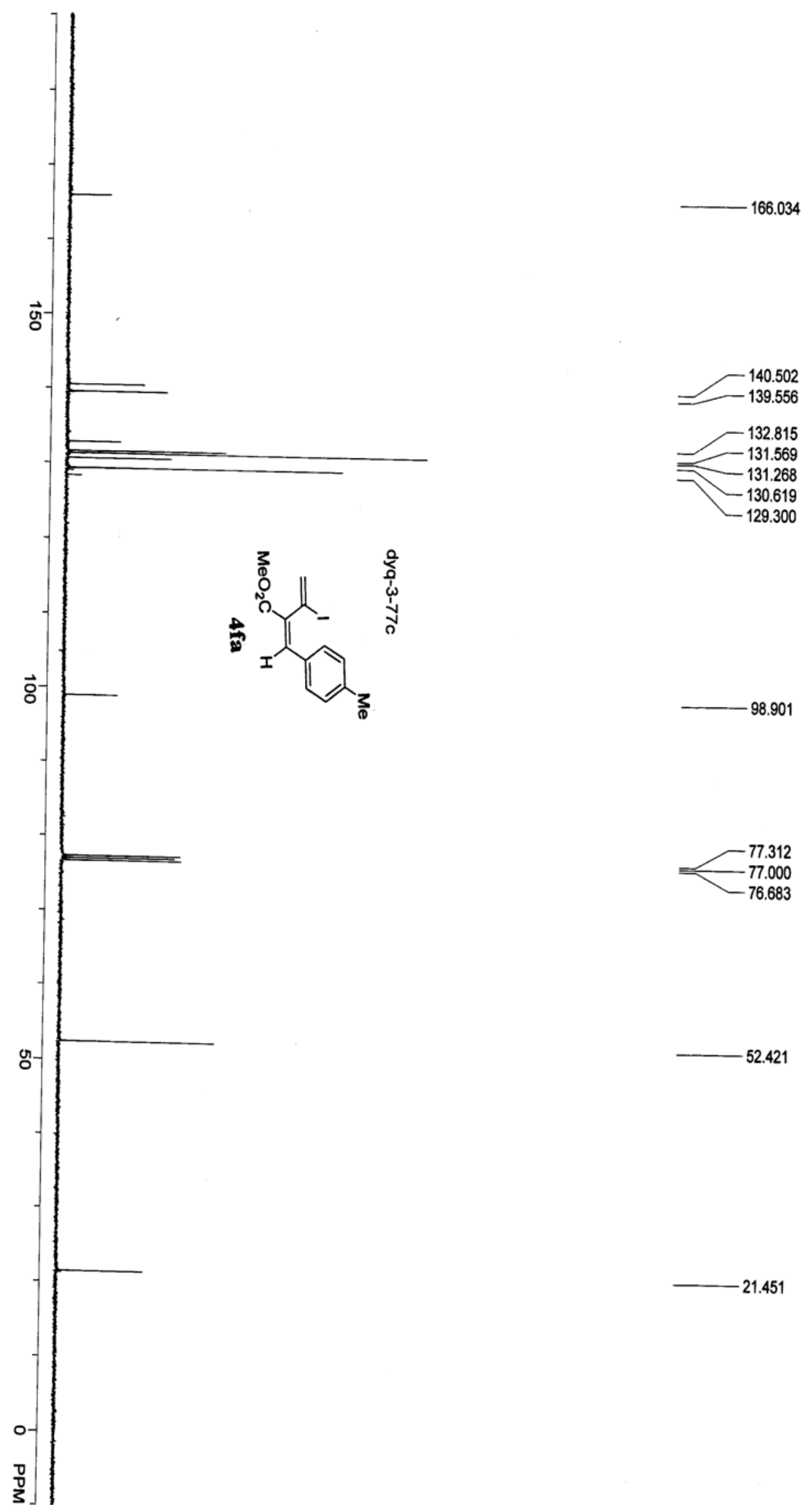

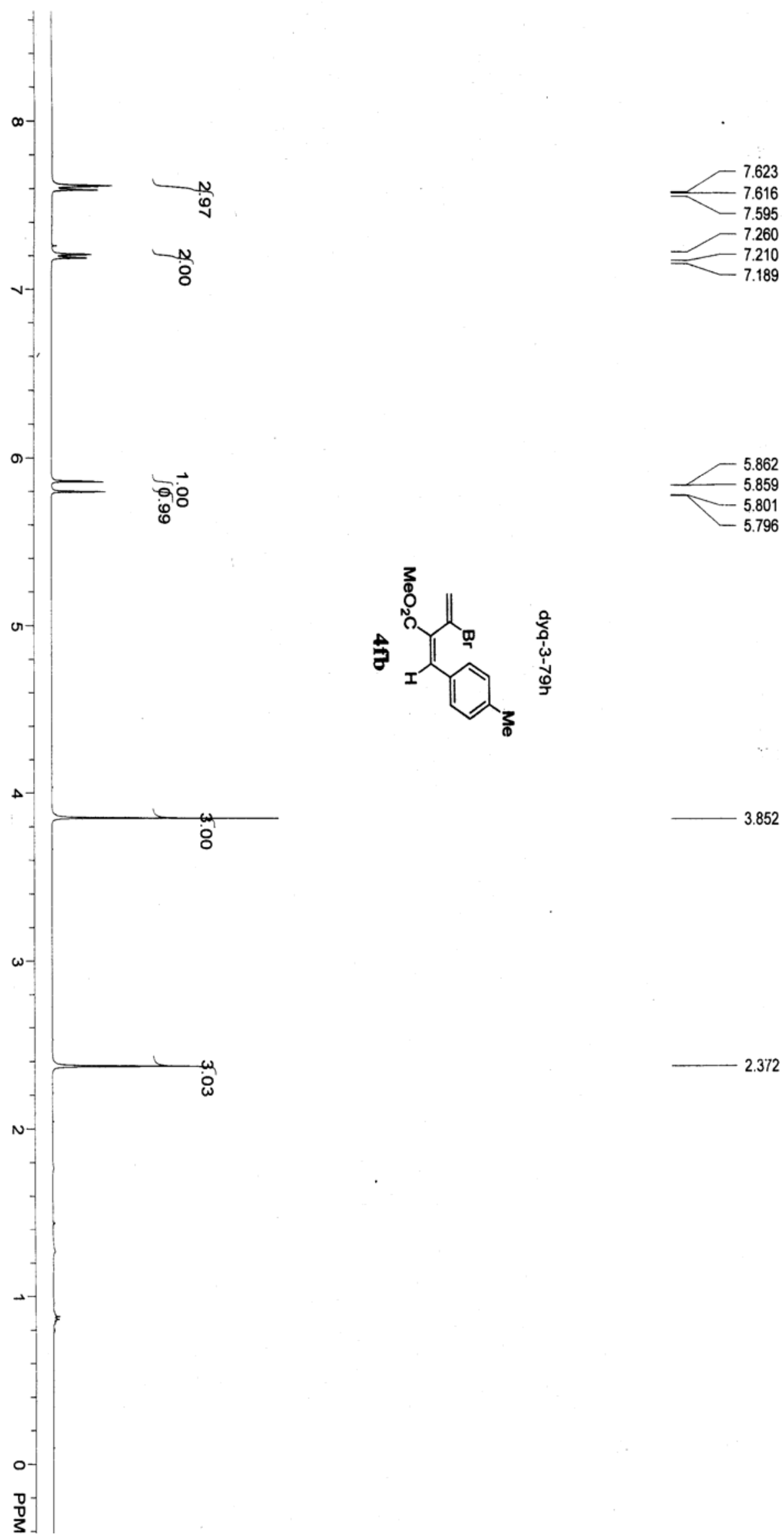

$-2.372$ 

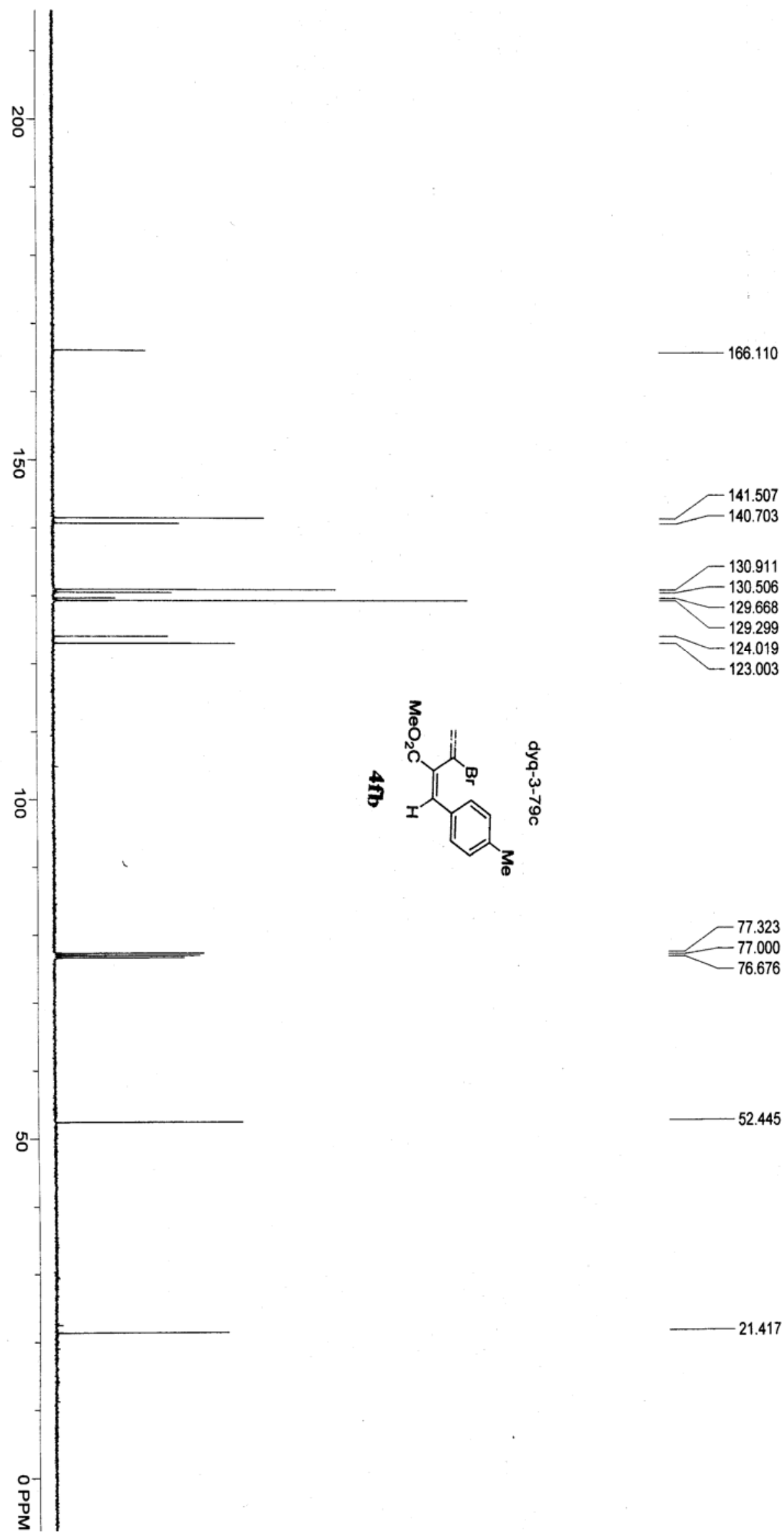

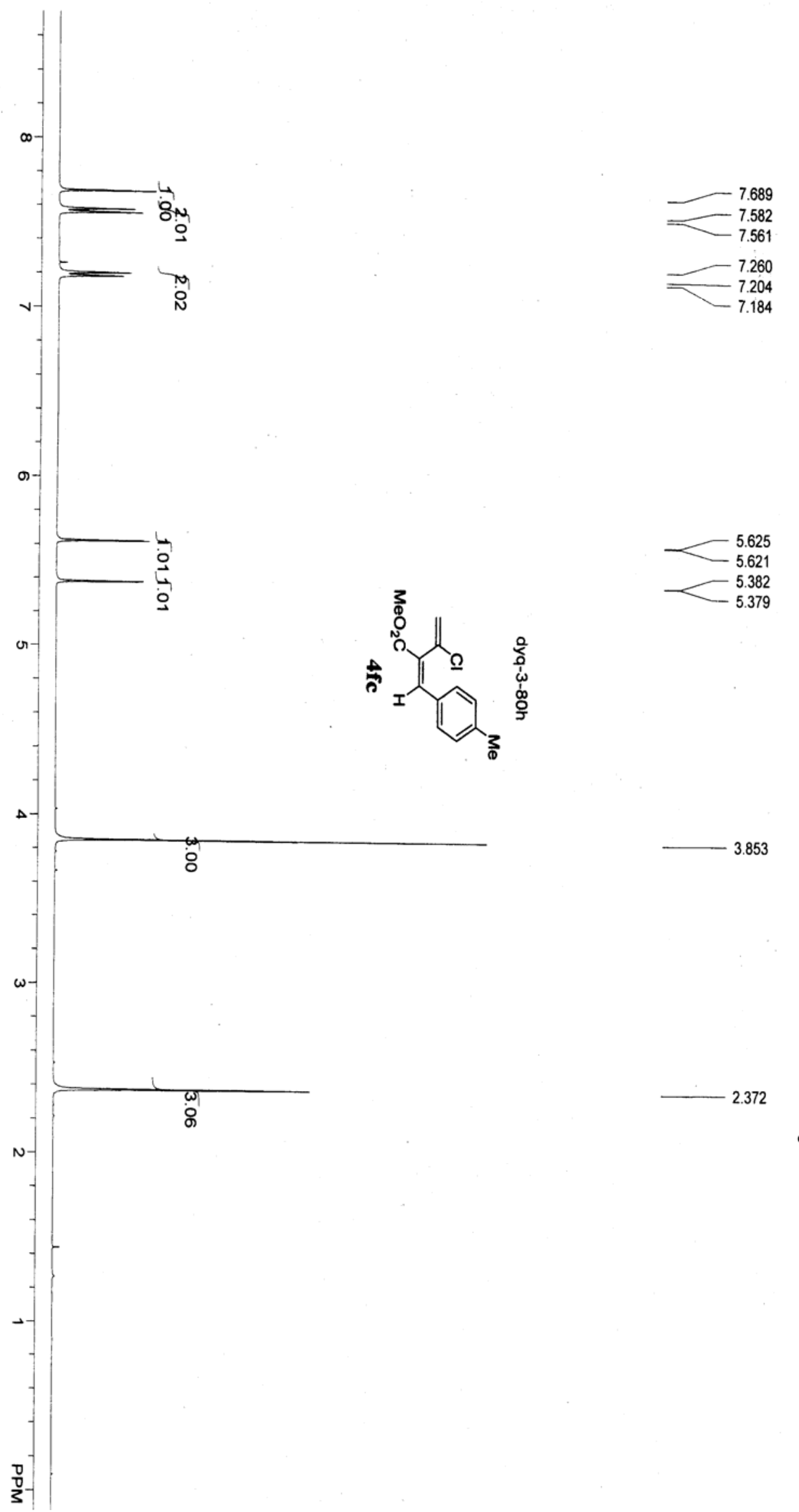

$-3.853$ 


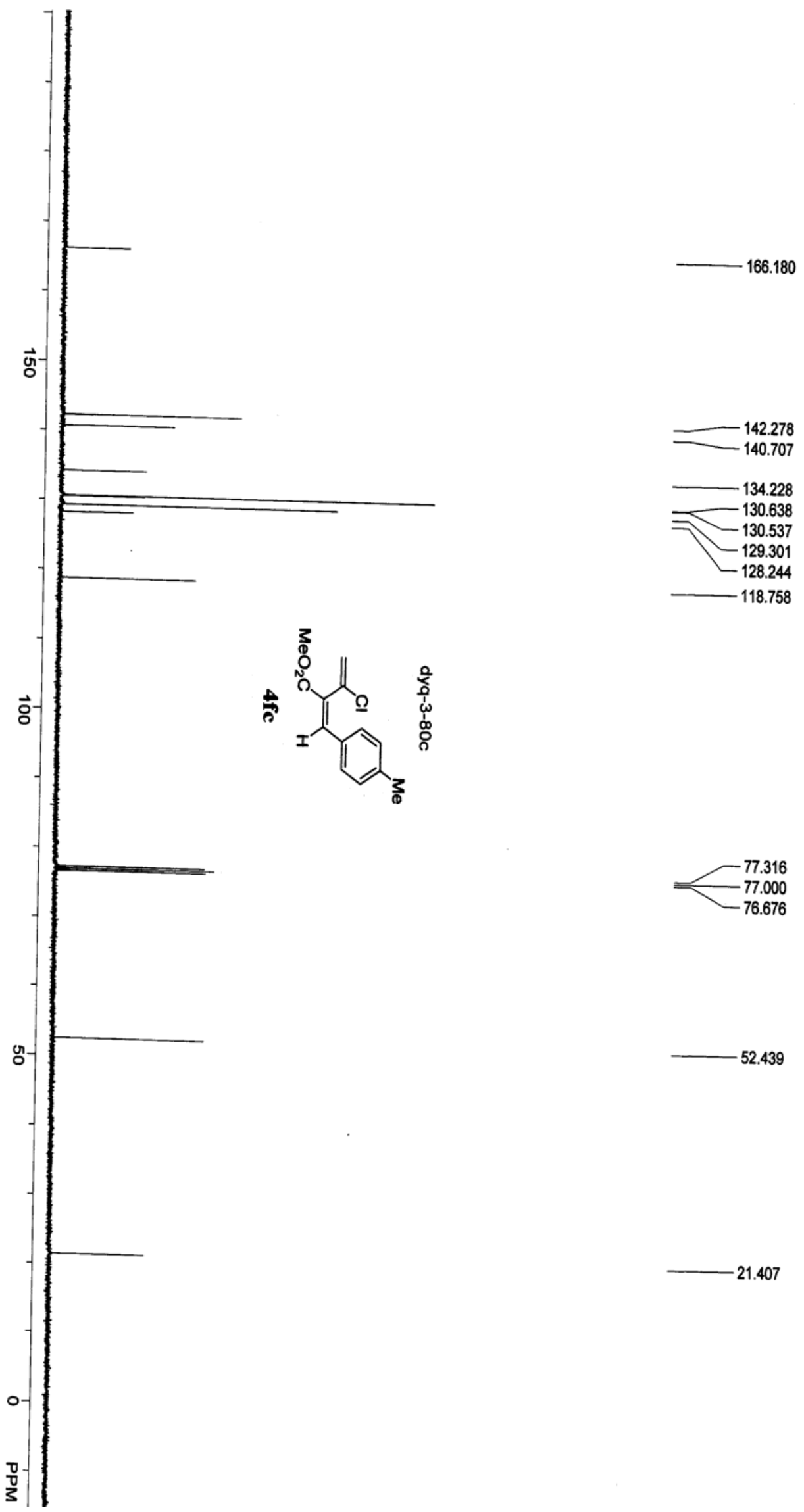



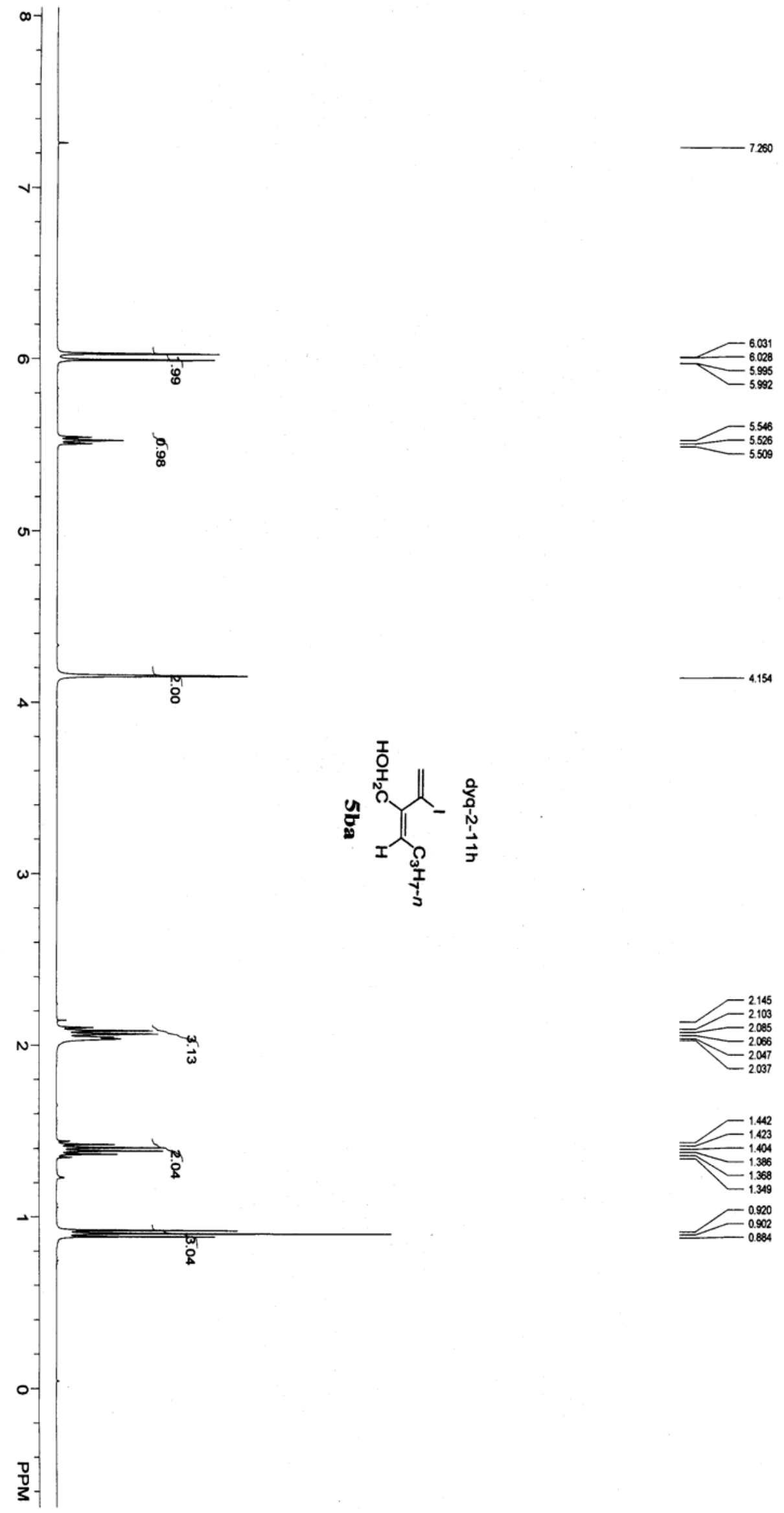

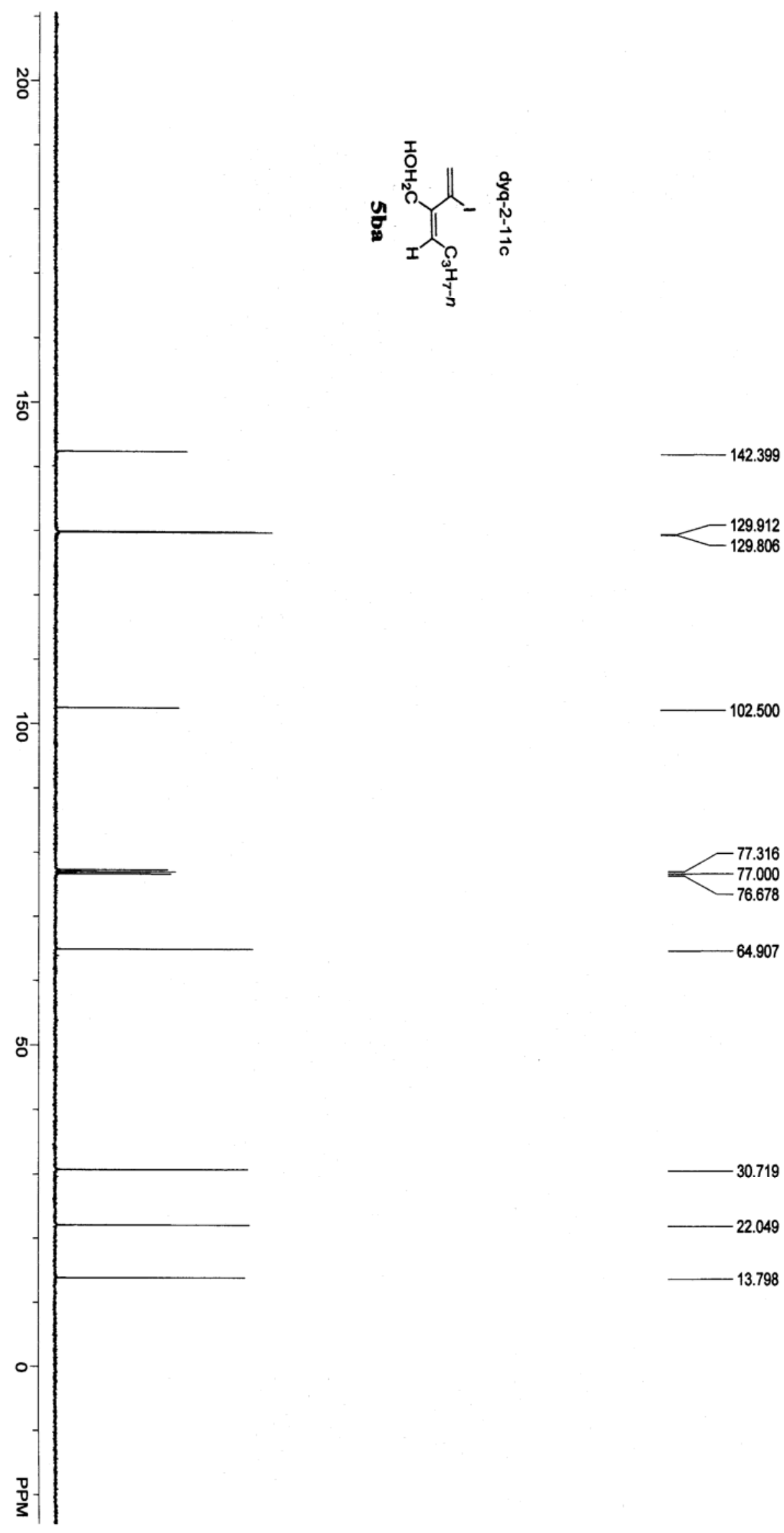


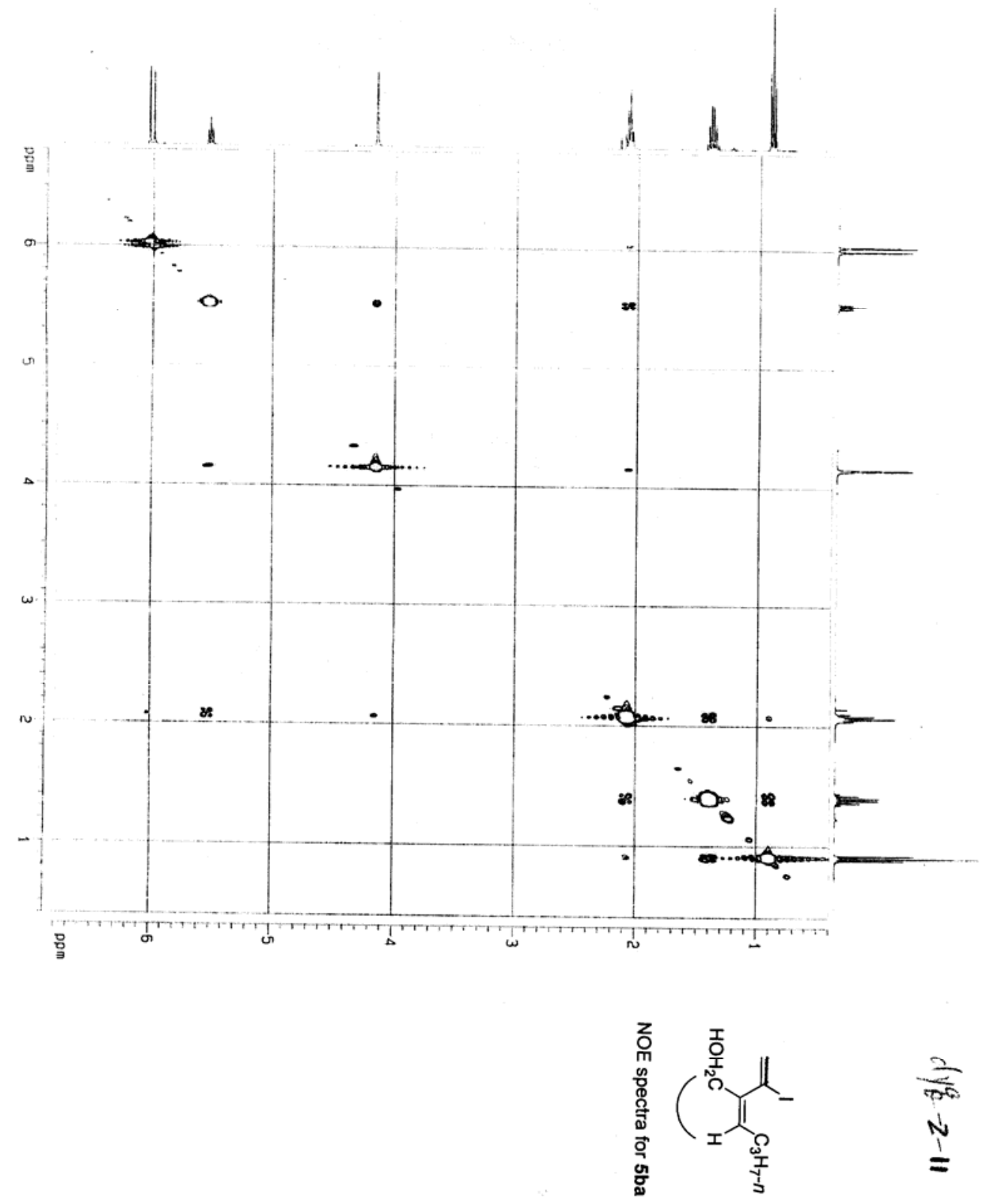



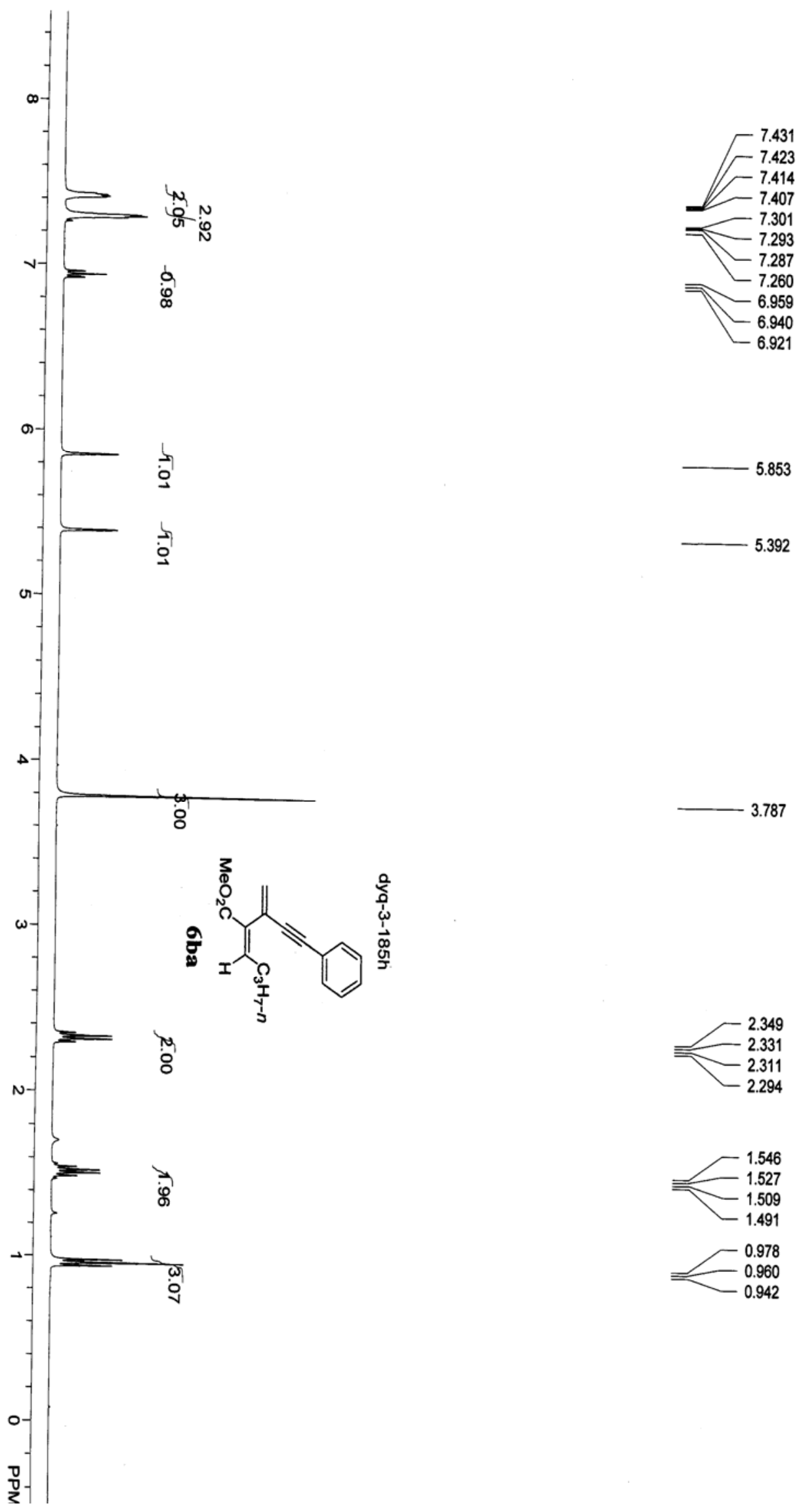

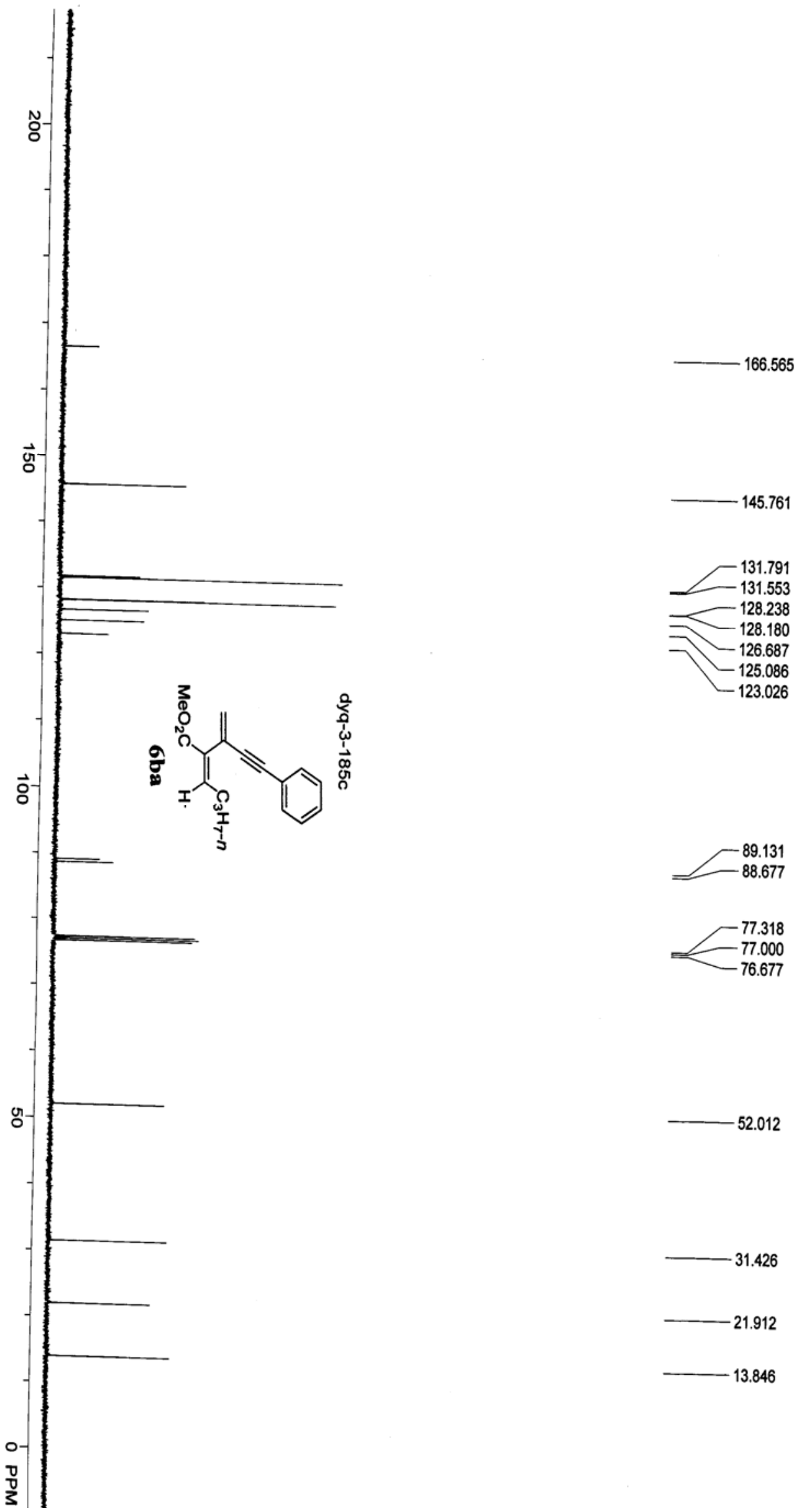


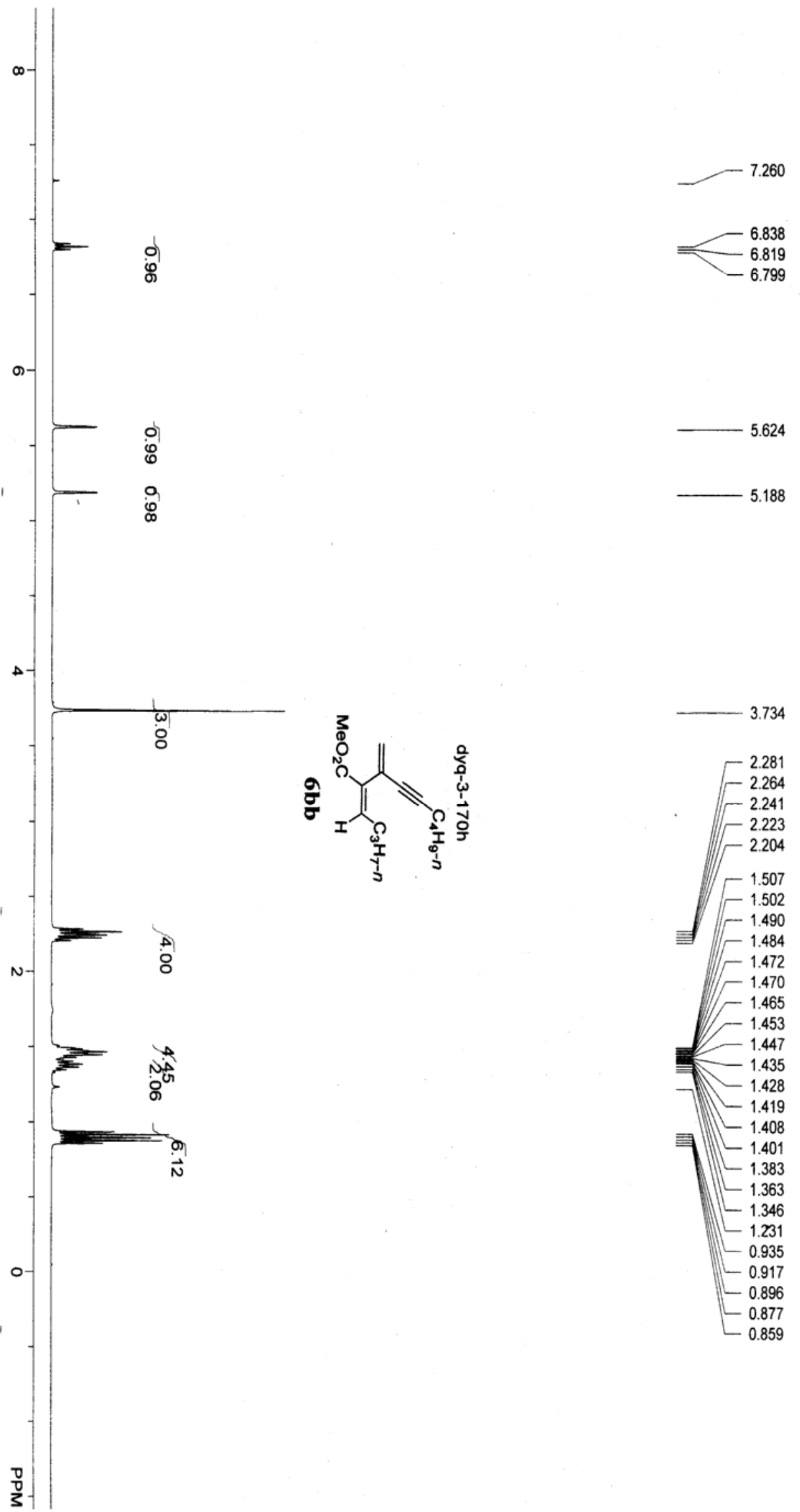




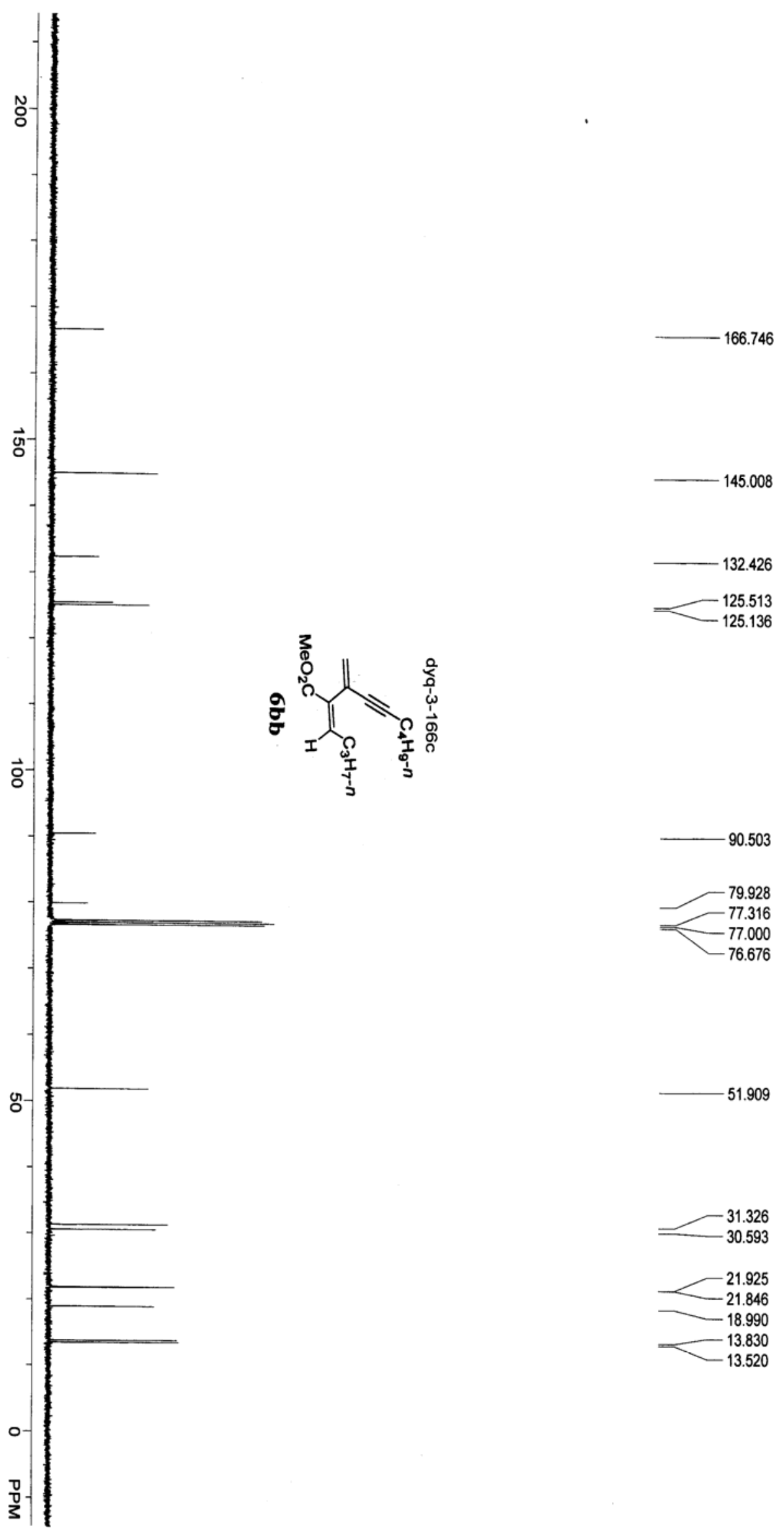




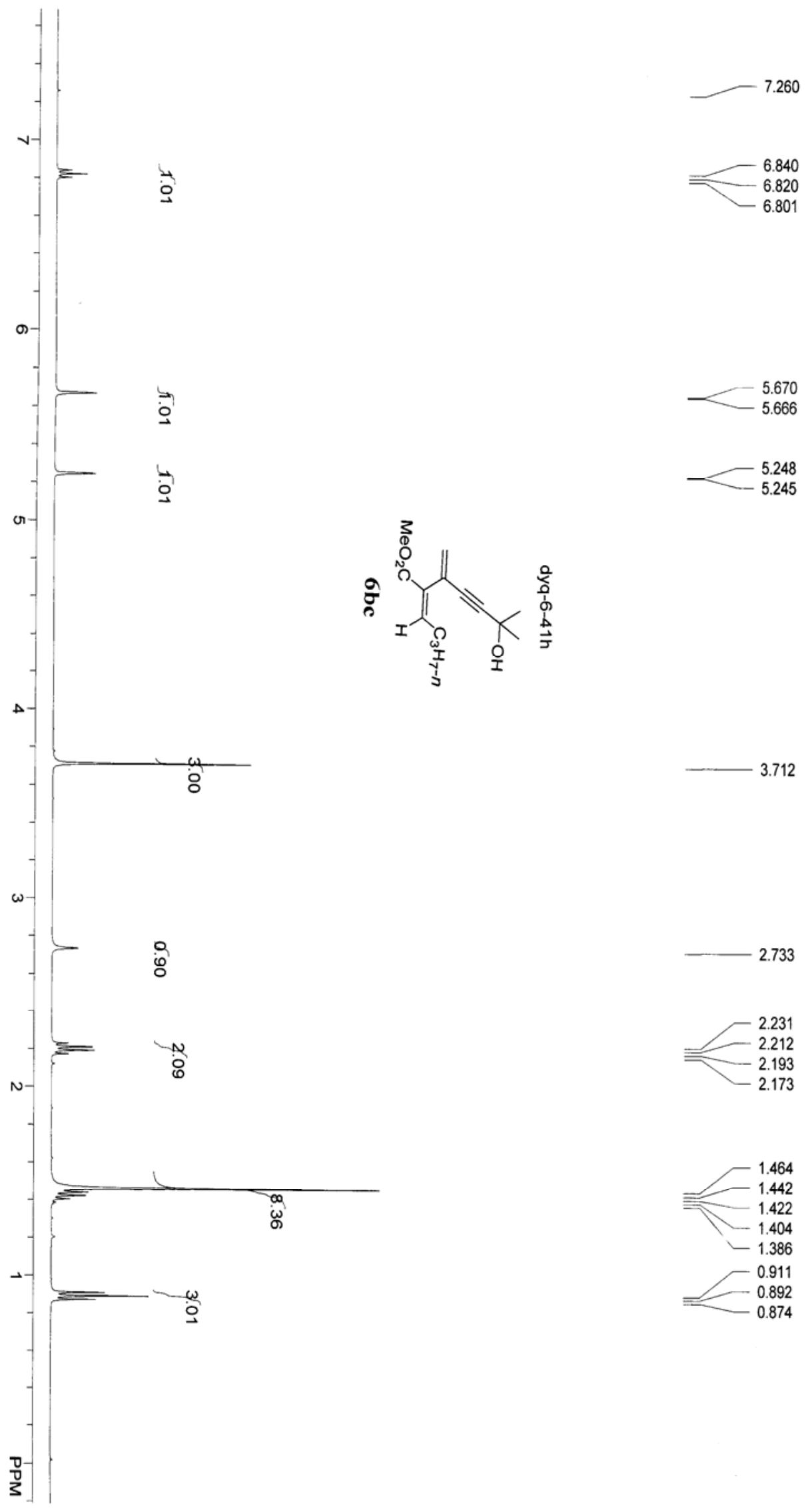




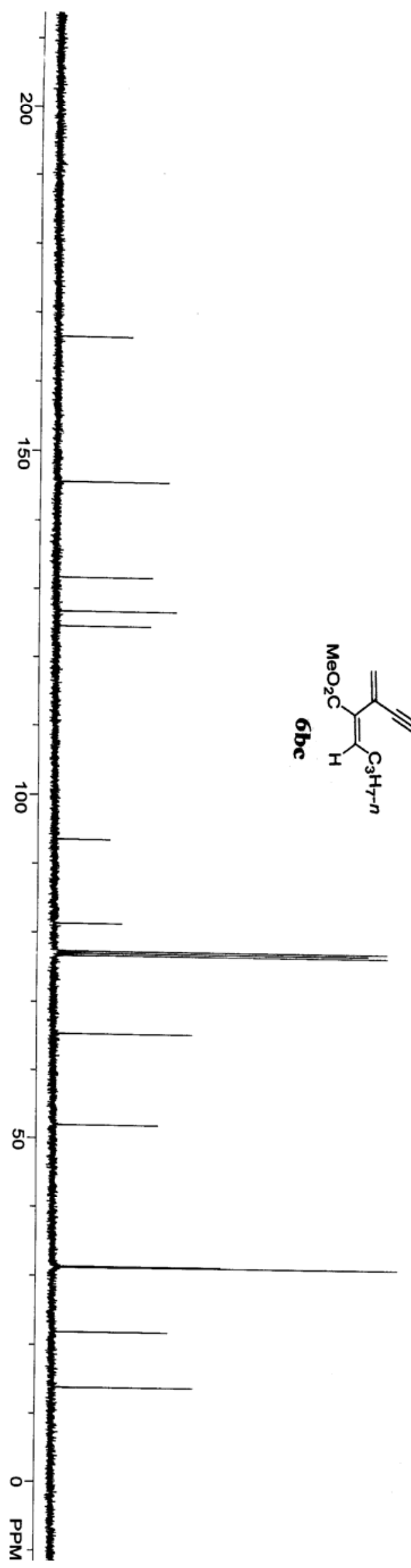

$-166.545$

145.646

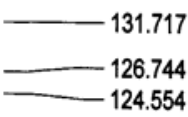

93.598
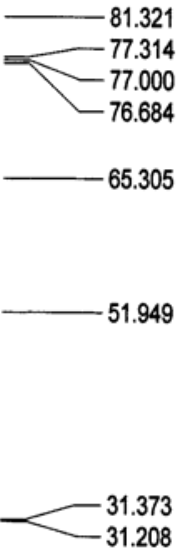

$-21.863$

$-13.826$ 

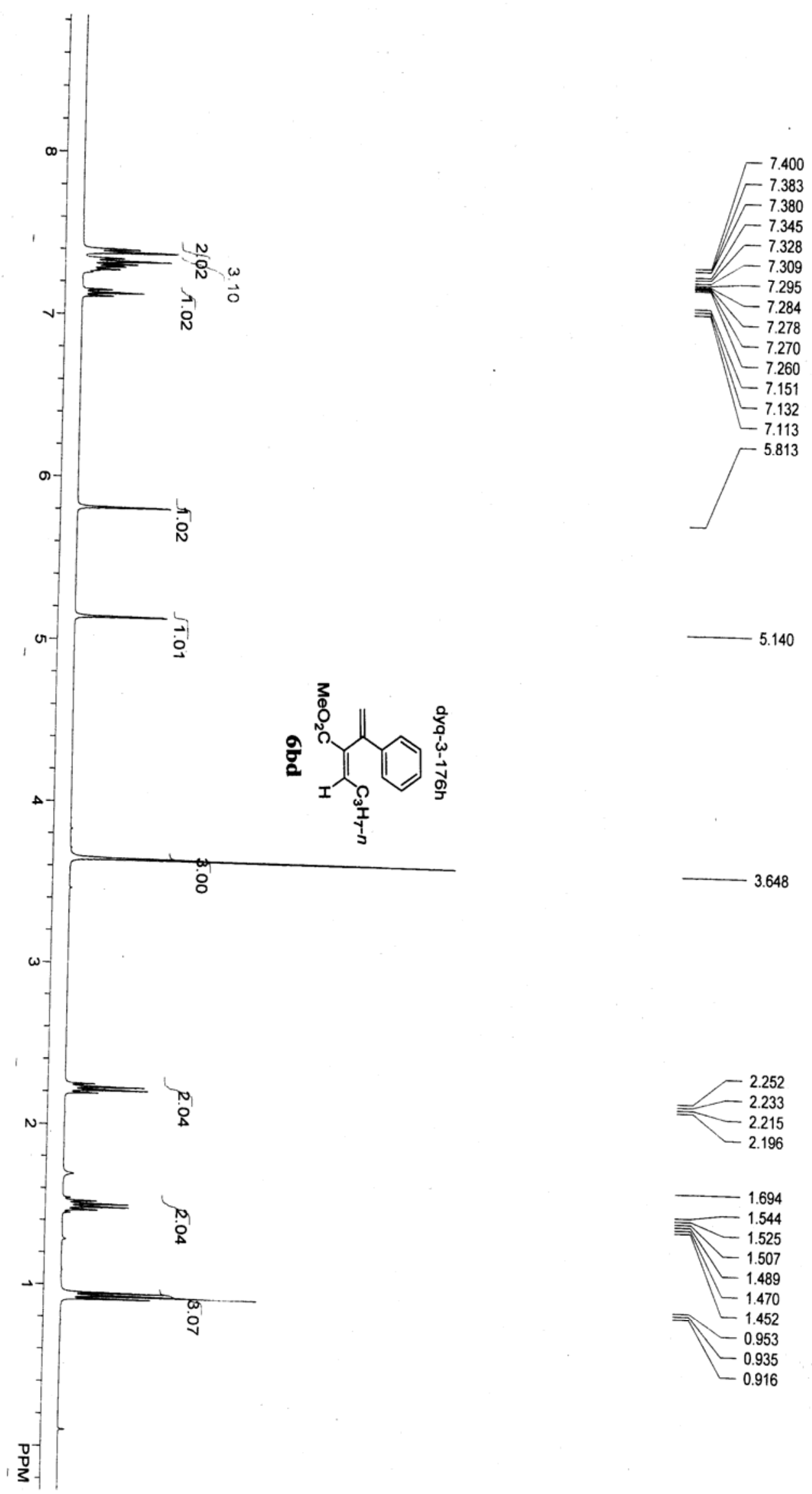

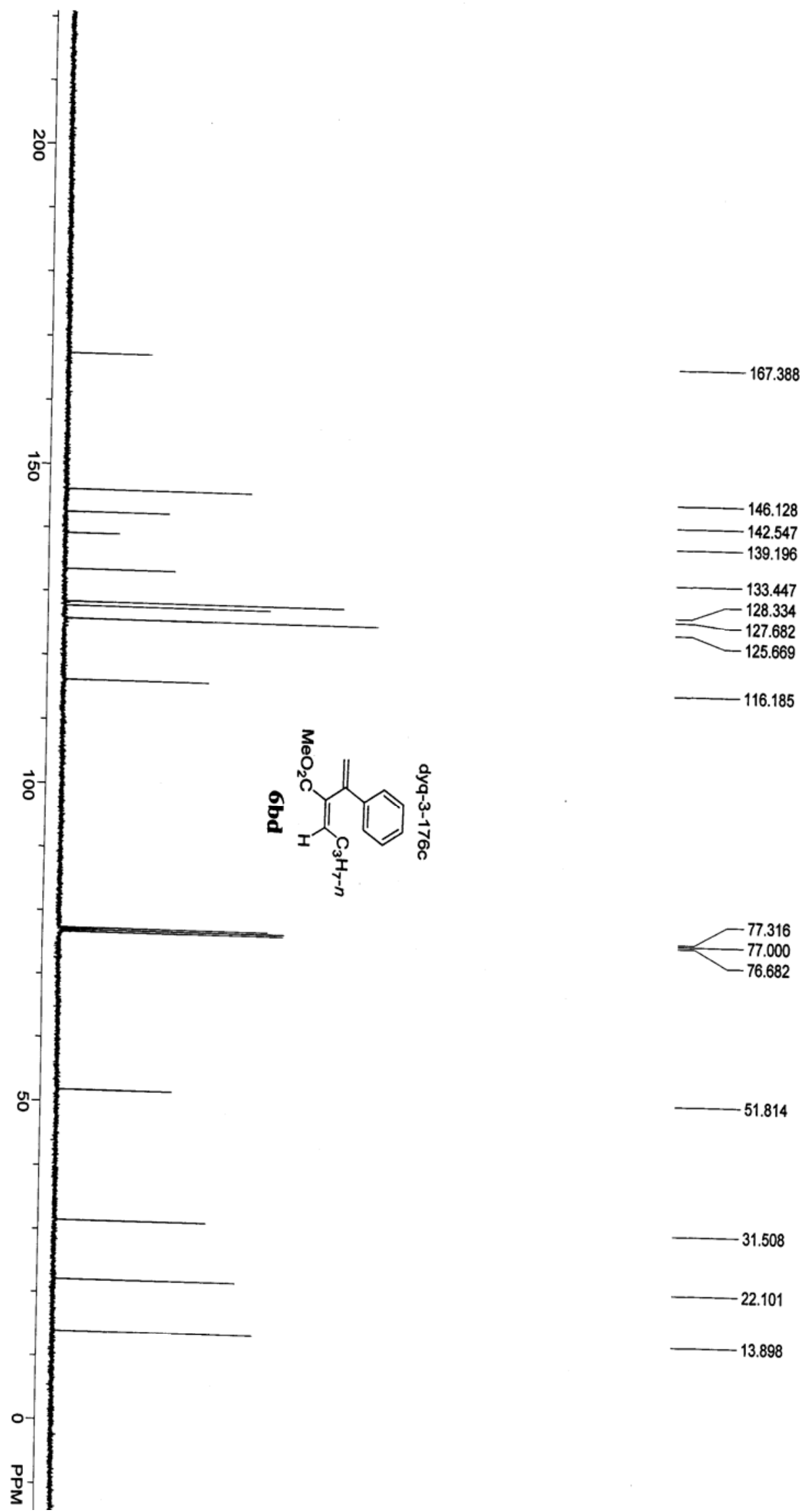

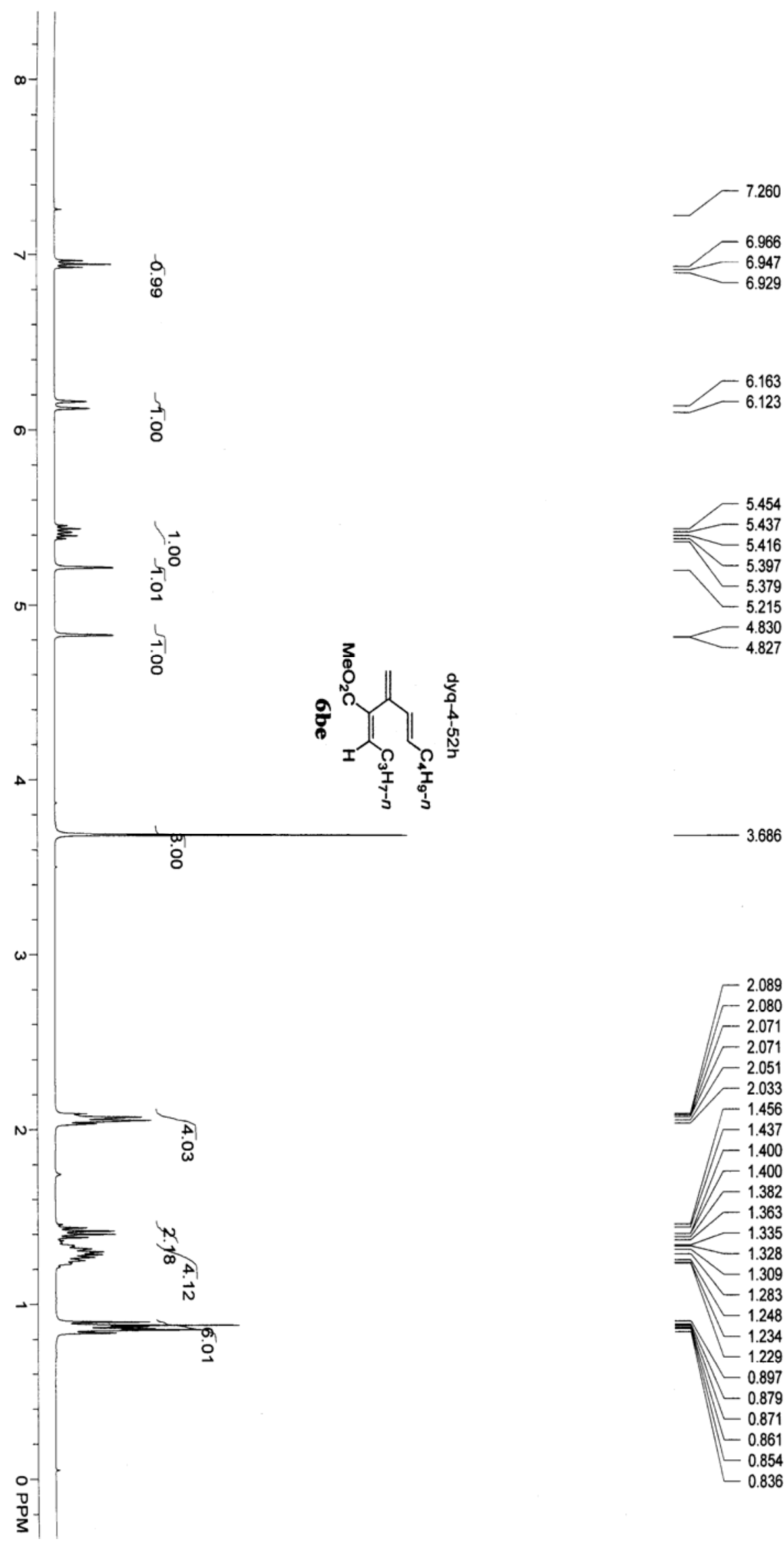


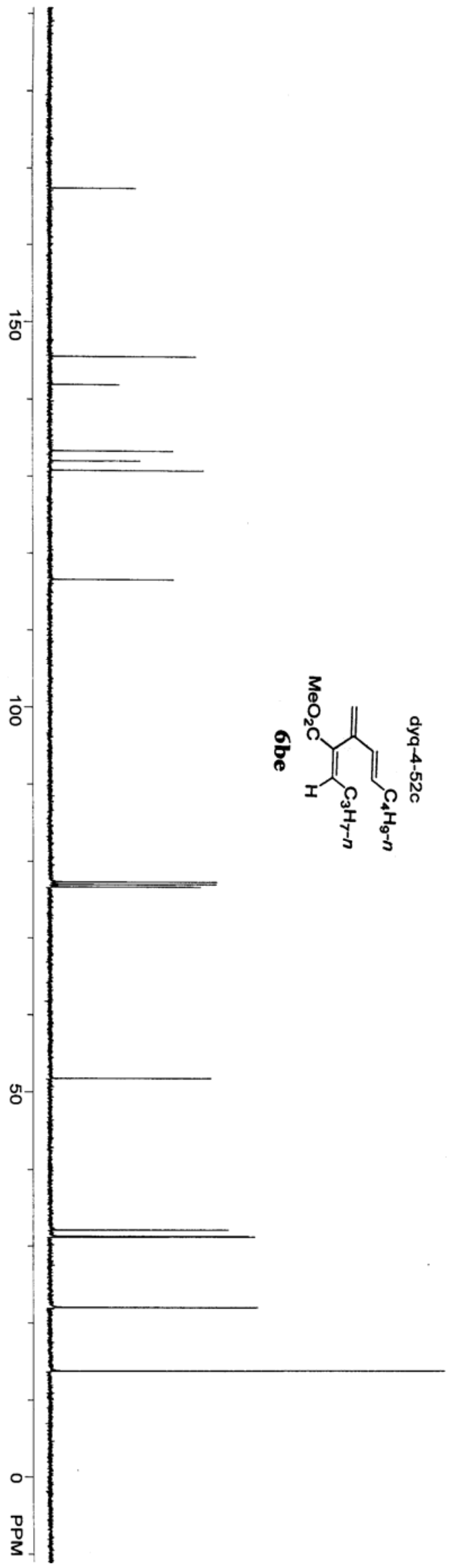

$-167.435$

145.532

141.900

133.319

$-132.029$

130.788

$-116.567$ 

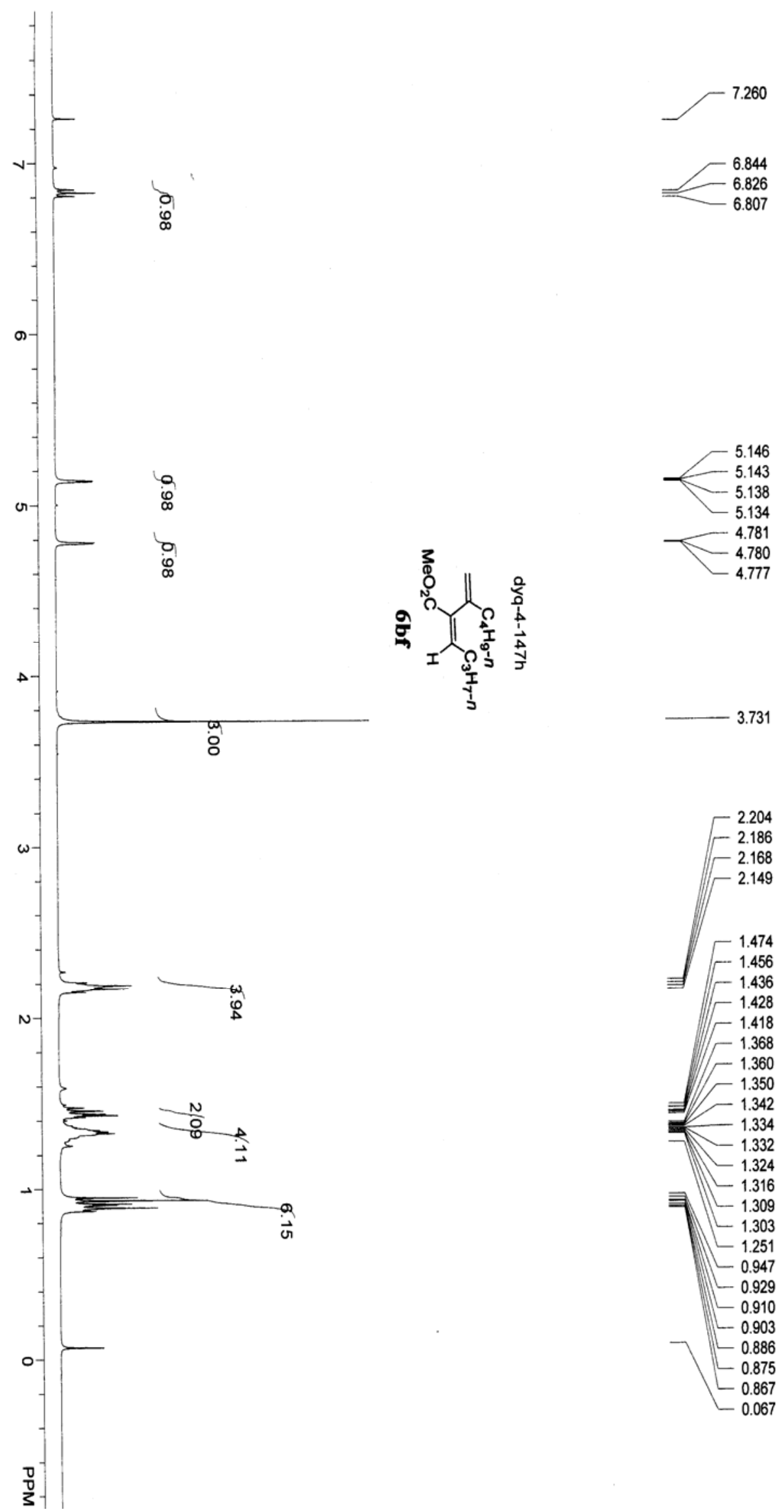


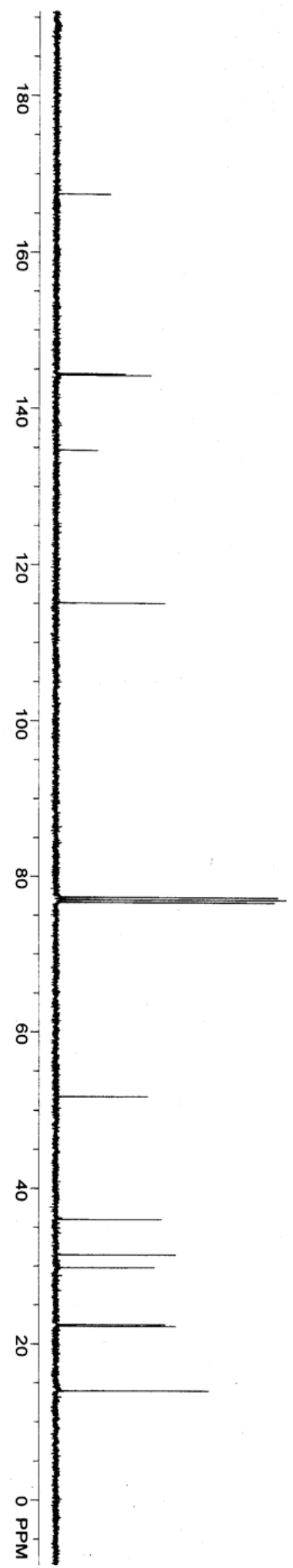

$-167.486$

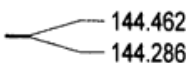

$-134.690$

115.099

A
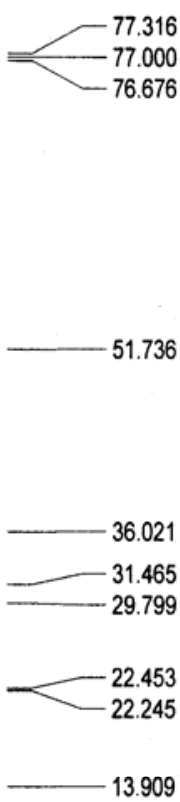